Aus der Abteilung Nephrologie und Rheumatologie

(Prof. Dr. med. G. A. Müller)

im Zentrum Innere Medizin

der Medizinischen Fakultät der Universität Göttingen

\title{
Das Schlafapnoe-Syndrom bei Dialysepatienten
}

\author{
INAUGURAL - DISSERTATION \\ zur Erlangung des Doktorgrades \\ der Medizinischen Fakultät \\ der Georg-August-Universität zu Göttingen
}

\author{
vorgelegt von \\ Oliver Weidler
}

aus

Cuxhaven

Göttingen 2010 
Dekan:

I. Berichterstatter:

II. Berichterstatter:

III. Berichterstatter:

Tag der mündlichen Prüfung:
Prof. Dr. med. C. Frömmel

Prof. Dr. med. G. A. Müller Priv.-Doz. Dr. med. Lüthje Prof. Dr. med. Oppermann

28.09.2010 


\section{Inhaltsverzeichnis}

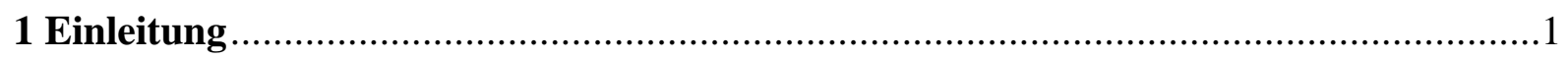

1.1 Definition Schlafbezogener Atemstörungen ......................................................2

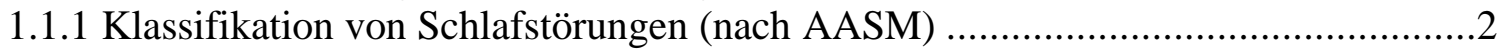

1.1.2 Definitionen Schlafbezogener Atemstörungen (nach ICSD) .................................4

1.1.2.1 Obstruktives Schlafapnoe-Syndrom (OSAS) ................................................4

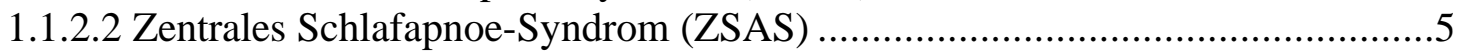

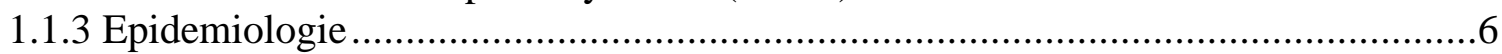

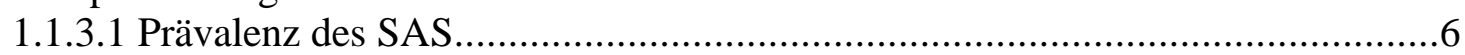

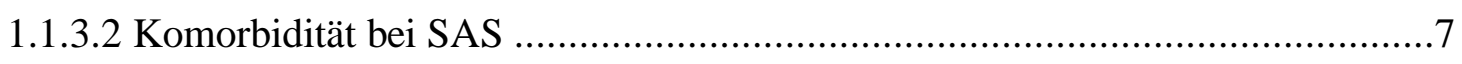

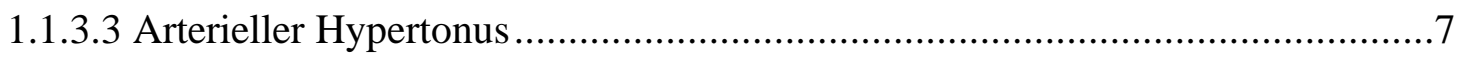

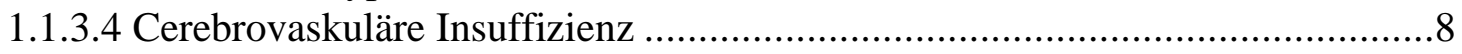

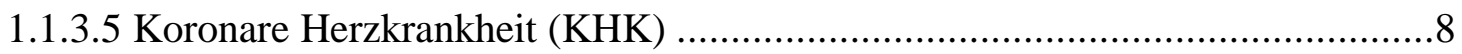

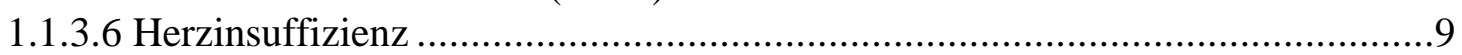

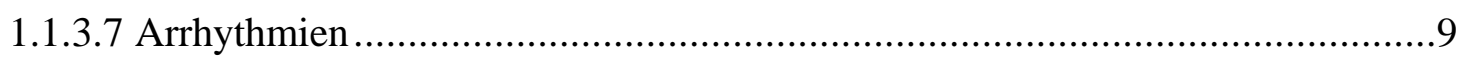

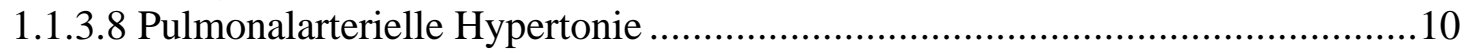

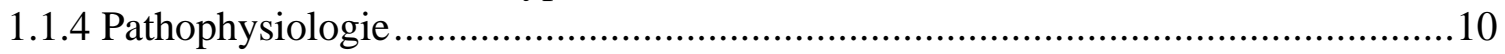

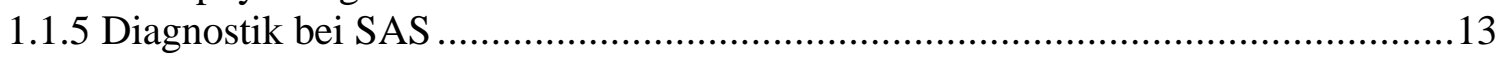

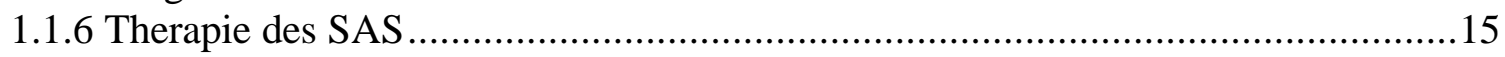

1.2 Terminale Niereninsuffizienz und chronische Nierenersatz-therapie ........................16

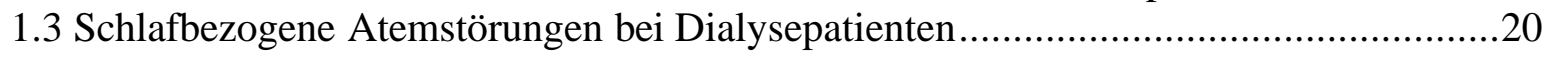

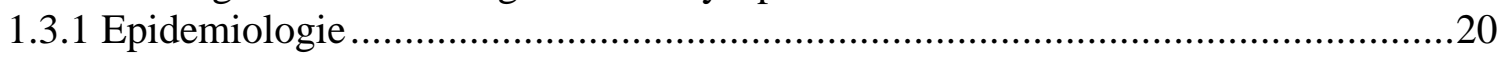

1.3.2 Spezielle Pathogenese und Therapie _..........................................................22

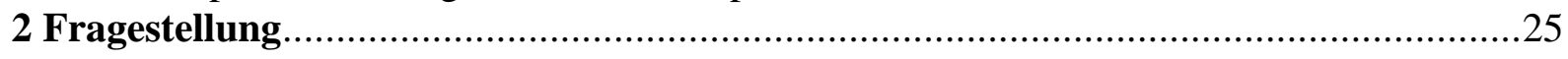

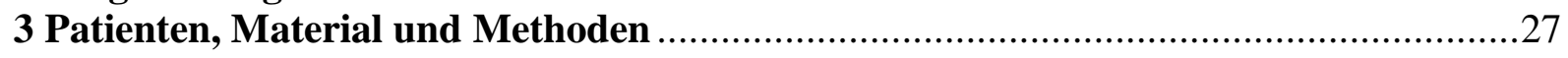

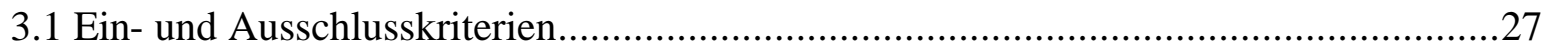

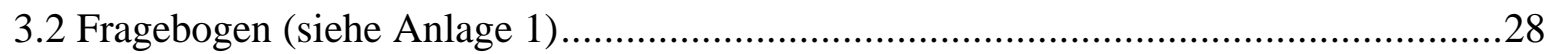

3.3 Durchführung des ambulanten Schlafapnoe-Screenings ..........................................29

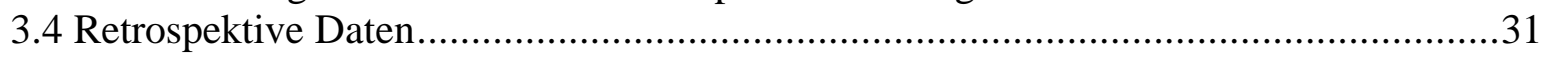

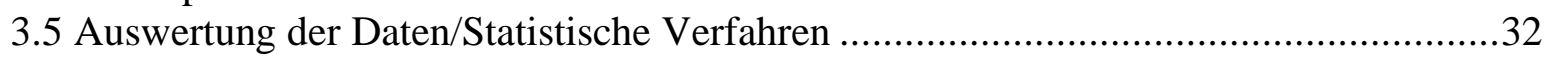

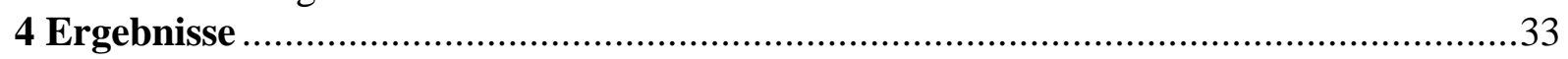

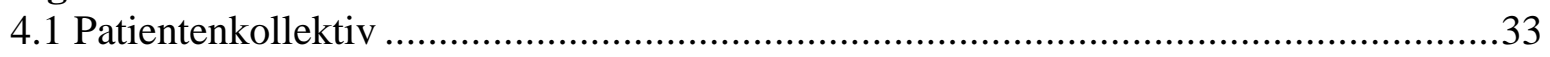

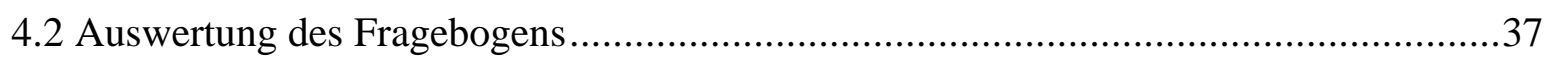

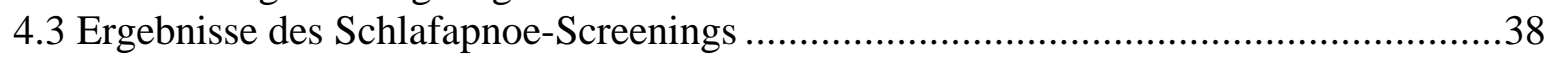

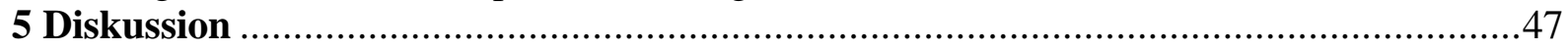

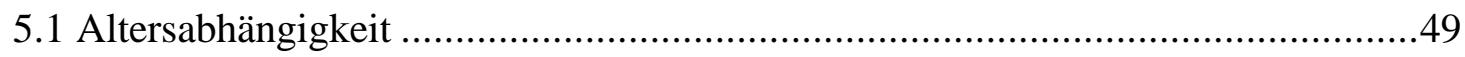

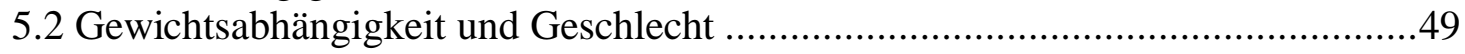

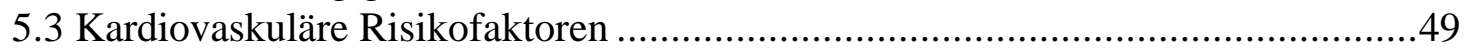

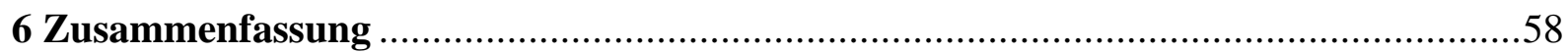

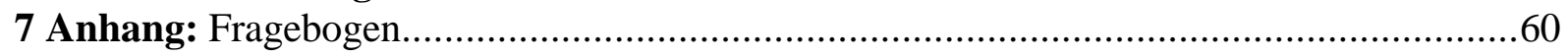

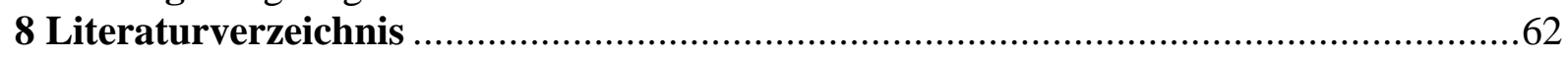




\section{Abkürzungsverzeichnis}

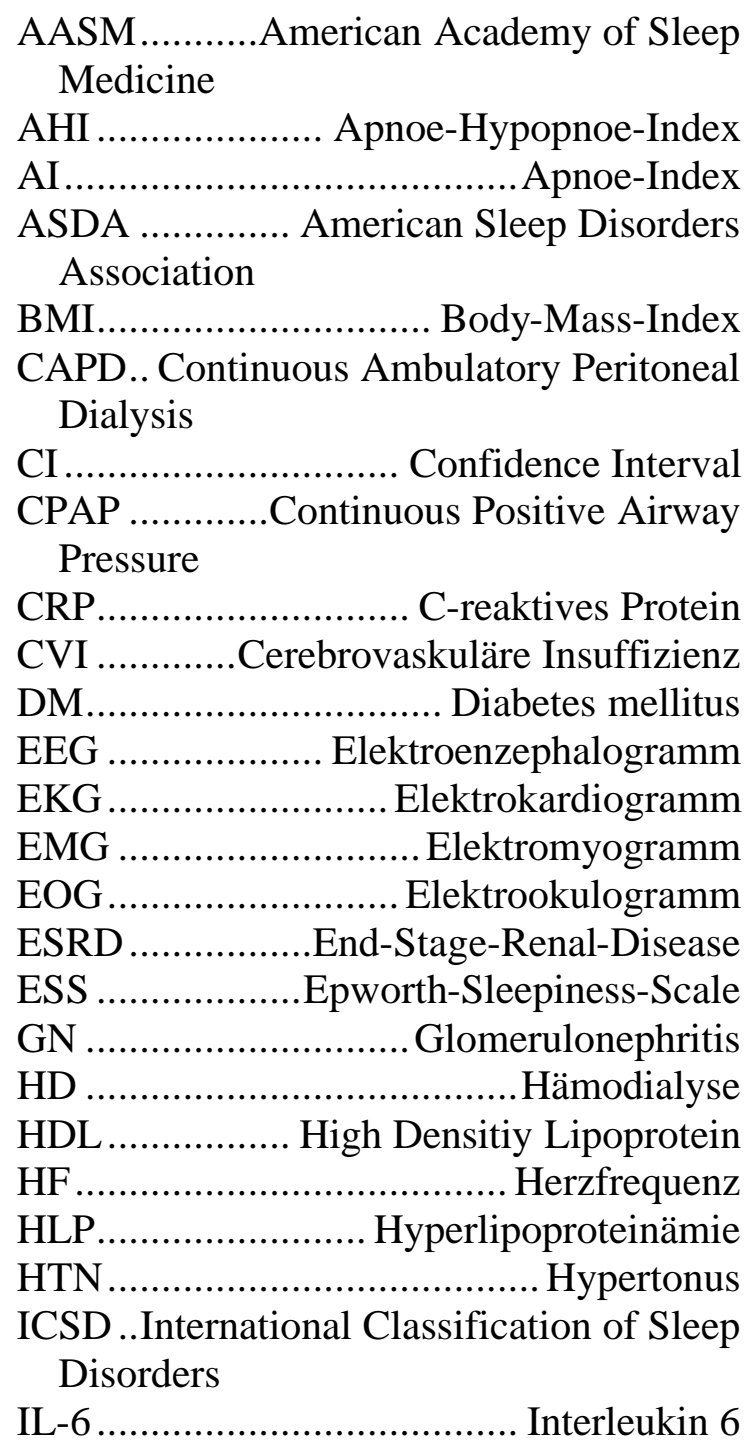

KHK Koronare Herzkrankheit

KVE Kardiovaskuläre Erkrankungen

LDL Low Densitiy Lipoprotein

LUPP Laser-Uvulopalatoplastik

MRSA Methicillin-resistant

Staphylococcus aureus

nCPAP nasales Continuous Positive

Airway Pressure

NSAR .... Nicht-Steroidale Antirheumatika

OR Odds Ratio

OSA Obstruktive Schlafapnoe

OSAS... Obstruktives Schlafapnoesyndrom PAP Pulmonal Arterial Pressure pAVK periphere Arterielle

\section{Verschlusskrankheit}

$\mathrm{PH}$ Pulmonaler Hypertonus

PSG Polysomnographie

RDI............Respiratory-Disturbance-Index

RLS .................... Restless-Legs-Syndrom

SAS ...................... Schlafapnoe-Syndrom SRBD .. Sleep Related Breathing Disorders TIA.....Transitorische Ischämische Attacke TNF-a .........Tumor-Necrosis-Factor-Alpha UKG .........Universitätsklinikum Göttingen UPPP ............Uvulopalatopharyngoplastik WHO ...............World Health Organisation ZNS ................... Zentrales Nervensystem ZSA ........................Zentrale Schlafapnoe ZSAS .......Zentrales Schlafapnoe-Syndrom 


\section{Einleitung}

Schlafbezogene Atemstörungen sind ein häufiges Phänomen in der Gesamtbevölkerung. Kommt es zu klinisch fassbaren Symptomen, spricht man vom Schlafapnoe-Syndrom (SAS). Die während des Schlafes auftretenden Ventilationsstörungen sind durch repetitive Apnoen und Hypopnoen von mindestens 10 Sekunden Dauer charakterisiert. Diese Phasen werden durch eine Weckreaktion ("arousal") terminiert und haben einen Abfall der Blutsauerstoffsättigung zur Folge.

Eine der ersten Beschreibungen eines typischen SAS-Patienten wurde 1836 von Charles Dickens in den „Pickwick Papers“ veröffentlicht. Er beschreibt den dicken Jungen Joe, der mit seiner Schläfrigkeit und Schnarchen zwei wichtige Kriterien der Schlafapnoe erfüllt und bei wohl vorliegender Polyzythämie (,,red faced“) und Herzinsuffizienz (,young dropsy“) sogar schon Langzeitfolgen der Schlafapnoe aufweist.

In Anlehnung an die Erzählungen wird das Vollbild des SAS als „Pickwick-Syndrom“ bezeichnet.

1976 werden die im Schlaf auftretenden Atemregulationsstörungen von Guilleminault als „Schlafapnoe-Syndrom“ bezeichnet und näher charakterisiert (Guilleminault et al., 1976).

Häufige „Arousals“ führen zur Schlaffragmentation und damit zur Störung der physiologischen Schlafarchitektur. Die betroffenen Patienten leiden typischerweise an exzessiver Tagesmüdigkeit und einer zum Teil massiv gesteigerten Neigung, auch in ungewöhnlichen Situationen einzuschlafen. Häufig schnarchen betroffene Patienten. Zusätzlich treten uncharakteristische Symptome, wie Konzentrationsstörungen, intellektuelle Beeinträchtigung, Kopfschmerzen und Potenzstörungen auf.

Das Schlafapnoe-Syndrom gilt als unabhängiger Risikofaktor für die Entstehung des arteriellen Hypertonus (Lavie P et al., 2000), der KHK (Hung et al., 1990), von cerebrovaskulären Insulten und der Herzinsuffizienz (Shahar et al., 2001). Es ist mit kardialen Arrhythmien (Guilleminault at al., 1983) und pulmonalem Hypertonus assoziiert (Bonsignore et al., 1994).

Chronisch niereninsuffiziente Patienten leiden unter Symptomen, die denen des SAS in vielerlei Hinsicht ähneln (Kimmel, 1989).

Die Prävalenz der Schlafapnoe bei Dialysepatienten liegt zwischen 53\% und 89\% (Hallett et al., 1995 und Wadhwa et Mendelson., 1992). Kardiovaskuläre Komplikationen sind mit $43 \%$ die häufigste Todesursache bei Dialysepatienten (Frei et Schober-Halstenberg, 2004). 
Es ist bekannt, dass das Schlafapnoe-Syndrom den Verlauf kardiovaskulärer Erkrankungen verschlechtert. Allerdings sind die Auswirkungen des SAS auf den Verlauf von kardiovaskulären Erkrankungen bei Dialysepatienten nicht untersucht.

Durch das SAS kann das Immunsystem beeinträchtigt werden (Benca et Quintas, 1997). Ob dies bei Dialysepatienten zu der klinisch relevanten Infektneigung beiträgt, ist ebenso nicht untersucht. Infektiöse Komplikationen stellen die zweithäufigste Todesursache bei Dialysepatienten dar (Frei et Schober-Halstenberg, 2004).

Den Behandlungsstandard bei SAS stellt heute die nicht invasive Continuous Positive Airway Pressure (CPAP) - Beatmung mittels Maske dar. Die Methode ist auch bei Dialysepatienten erfolgreich anwendbar (Pressman et al., 1993).

Es liegen gegenwärtig keine Interventionsstudien vor, die die klinische Relevanz des Schlafapnoesyndroms beziehungsweise die Effektivität der Behandlung bei niereninsuffizienten Patienten belegen.

Ziel der vorliegenden Studie ist die Ermittlung der Prävalenz des Schlafapnoe-Syndroms bei den Dialysepatienten der Station 1011 des Universitätsklinikums Göttingen. Zur studieninternen Diagnosestellung wird ein ambulantes Schlafapnoe-Screening durchgeführt. Die Anwendbarkeit klinischer diagnostischer Kriterien bei Dialysepatienten wird überprüft. Eine Korrelation von etablierten kardiovaskulären Risikofaktoren soll hergestellt werden. Ebenso sollen Hinweise auf einen möglichen Zusammenhang des SAS mit den routinemäßig erhobenen Infektparametern ermittelt werden.

\subsection{Definition Schlafbezogener Atemstörungen}

\subsubsection{Klassifikation von Schlafstörungen (nach AASM)}

1990 entwickelte die American Academy of Sleep Medicine (AASM, 1990) in Zusammenarbeit mit der European Sleep Research Society, der Japanese Society of Sleep Research und der Latin American Sleep Society die internationale Klassifikation der Schlafstörungen (ICSD, International Classification of Sleep Disorders). Diese ist hier im Weiteren Grundlage für die Einteilung und Definition der Schlafstörungen. 
Tabelle 1 bietet einen Überblick über das ICSD-System (nach AASM, 1990):

\begin{tabular}{|c|c|}
\hline $\begin{array}{l}\text { Überblick über die Klassifikation der } \\
\text { Schlafstörungen nach ICSD }\end{array}$ & Definitionen \\
\hline $\begin{array}{l}\text { 1. Dyssomnien } \\
\text { A. Intrinsische Schlafstörungen } \\
\text { B. Extrinsische Schlafstörungen } \\
\text { C. Störungen des zirkadianen (Schlaf-) Rhythmus }\end{array}$ & $\begin{array}{l}\text { Schlafquantität und/ oder - } \\
\text { qualität sind beeinflusst. } \\
\text { Hauptmanifestationen: } \\
\text { Insomnien und/oder } \\
\text { Hypersomnien. }\end{array}$ \\
\hline $\begin{array}{l}\text { 2. Parasomnien } \\
\qquad \begin{array}{l}\text { A. Aufwachstörungen } \\
\text { (Arousal-Störungen) } \\
\text { B. Störungen des Schlaf-Wach-Übergangs } \\
\text { C. REM-Schlaf-assoziierte Parasomnien } \\
\text { D. Andere Parasomnien }\end{array}\end{array}$ & $\begin{array}{l}\text { Episodische Unterbrechungen } \\
\text { des Schlafes durch } \\
\text { ungewöhnliche körperl. } \\
\text { Phänomene und } \\
\text { Verhaltensweisen(z.B. Schlaf- } \\
\text { wandeln). }\end{array}$ \\
\hline $\begin{array}{l}\text { 3. Schlafstörungen bei körperlichen/psychiatrischen } \\
\text { Erkrankungen } \\
\text { A. Bei psychischen Störungen } \\
\text { B. Bei neurologischen Erkrankungen } \\
\text { C. Bei anderen körperlichen Erkrankungen }\end{array}$ & $\begin{array}{l}\text { Sek. Schlafstörungen, } \\
\text { verursacht durch körperl. oder } \\
\text { psychiatr. Erkrankungen. }\end{array}$ \\
\hline
\end{tabular}

$\mathrm{Zu}$ den Dyssomnien werden die intrinsischen Schlafstörungen gerechnet, die ihren Ursprung im Organismus haben. Sie sind unabhängig von äußeren Einflüssen. Externe Faktoren können allerdings bei der Entstehung eine Rolle spielen.

In diese Gruppe gehören u.a. das obstruktive Schlafapnoe-Syndrom (OSAS), das zentrale Schlafapnoe-Syndrom (ZSAS) und das zentrale alveoläre Hypoventilationssyndrom. Zusammenfassend werden diese Schlafstörungen auch als schlafbezogene Atemstörungen (Sleep Related Breathing Disorders, SRBD) bezeichnet. 


\subsubsection{Definitionen Schlafbezogener Atemstörungen (,Sleep related breathing disorders“, SRBD) nach ICSD (AASM, 1990)}

Apnoe: Als Apnoe werden vollständige Atmungsstillstände mit mindestens 10 Sekunden Dauer bezeichnet. Folge sind Sauerstoffsättigungsabfälle des Blutes.

Hypopnoe: Hypopnoen sind partielle respiratorische Obstruktionen während des Schlafes. Es kommt dabei zu Verringerung des Atemluftflusses von über $50 \%$ von mindestens 10 Sekunden Dauer und dadurch zu Sauerstoffsättingungsabfällen des Blutes.

Der Apnoe-Hypopnoe-Index (AHI)/Respiratory-Disturbance-Index (RDI) ist als Summe von Apnoen und Hypopnoen pro Stunde während des Schlafes definiert. Damit ist eine Einteilung der Schwere der vorliegenden Schlafapnoe möglich (normal: AHI weniger als 5; mild: 5-15; mittelschwer: 15-30; schwer: über 30) (AASM, 1999).

\subsubsection{Obstruktives Schlafapnoe-Syndrom (OSAS)}

Nach ICSD ist das obstruktive Schlafapnoe-Syndrom durch wiederholte obstruktive Episoden der oberen Atemwege gekennzeichnet, die während des Schlafes auftreten und mit einem Absinken des Blutsauerstoffgehalts einhergehen. Während dieser Phasen ist über Mund und Nase kein Luftfluss vorhanden, wobei jedoch, im Gegensatz zum zentralen Schlafapnoesyndrom (s.u.), thorakale und diaphragmale Atemexkursionen nachweisbar sind. Zur Diagnosestellung sind mindestens 5 Apnoephasen pro Stunde von jeweils mindestens 10 Sekunden Dauer notwendig. Klinisch imponiert das OSAS durch lautes Schnarchen mit Pausen von 20 bis 30 Sekunden Länge. Am Ende der Apnoephasen kommt es zu Weckreaktionen (,arousals“), die mit auffälligen Körperbewegungen einhergehen können.

Betroffene Patienten klagen über morgendliche Unausgeschlafenheit, Desorientiertheit und geistige Schwerfälligkeit. Morgendlicher Kopfschmerz und Mundtrockenheit werden ebenfalls berichtet. Ein Leitsymptom stellt die ausgeprägte Tagesmüdigkeit dar, die zu Problemen im Berufs- und Familienleben führen kann und zu einer erhöhten Unfallgefahr führt (Findley et al., 1992). Ferner ist das OSAS mit Übergewicht, Libidoverlust, Erektionsstörungen, gastroösophagealem Reflux und sekundären Depressionen assoziert. Systemischer und pulmonaler Hypertonus stehen mit dem OSAS im Zusammenhang, ebenso wie verschiedene kardiale Arrhythmien (ICSD). 


\subsubsection{Zentrales Schlafapnoe-Syndrom (ZSAS)}

Beim zentralen Schlafapnoe-Syndrom (ZSAS) kommt es zu Atemstillständen, die durch simultanes Sistieren der thorakalen und abdominalen Atemexkursionen sowie des nasalen und oralen Luftflusses charakterisiert sind. Sie werden durch ein Ausbleiben des zentralen Antriebs verursacht. Es kommt zum Absinken der Blutsauerstoffsättigung.

Zur Diagnosestellung sind mindestens 5 Apnoephasen pro Stunde von jeweils mindestens 10 Sekunden Dauer nötig.

Die Symptomatik ist durch nächtliche Schlaflosigkeit charakterisiert, die durch häufiges Erwachen mit dem Gefühl der Luftnot und des Erstickens verursacht wird. Die Folgen des ZSAS am Tage decken sich weitestgehend mit denen des OSAS. Im Gegensatz zum OSAS finden sich jedoch häufiger Störungen von Gedächtnis und anderen kognitiven Funktionen. Ein Schnarchgeräusch kann auftreten, ist aber seltener als beim OSAS. Es bestehen ebenfalls Assoziationen zu kardiovaskulären Erkrankungen.

Da beide Formen der Schlafapnoe mit den Folgen der nächtlichen Sauerstoffsättigungsabfälle und der exzessiven Tagesmüdigkeit einhergehen, ist das ZSAS aufgrund der ähnlichen Symptomatik klinisch oft nicht vom OSAS zu unterscheiden. Lautes Schnarchen kann ein wichtiger Hinweis auf eine eher obstruktive Komponente des SAS sein. Zur sicheren Unterscheidung zwischen beiden Apnoeformen dient die Polysomnographie (s.u.). Hierbei werden beim OSAS gemischte Apnoe-Phasen beobachtet. Diese sind durch eine initiale zentrale Komponente (Abwesenheit von Atemexkursionen) und eine sich anschließende obstruktive Komponente charakterisiert. 


\subsubsection{Epidemiologie}

\subsubsection{Prävalenz des SAS}

Verschiedene epidemiologische Studien zur Prävalenz der schlafbezogenen Atemstörungen (SRBD) zeigten unterschiedliche Ergebnisse. Dies ist durch Unterschiede im Studiendesign, unterschiedliche Studienpopulationen sowie Unterschiede in der Anwendung diagnostischer Kriterien für das SAS zu erklären.

Lavie P (1983) untersuchte 78 Arbeiter polysomnographisch. Die Prävalenz einer Schlafapnoe betrug 0,89\%. 11 Arbeiter hatten einen Apnoe-Index (AI) größer 10. Diese Probanden wurden aus einem Kollektiv von 1236 Arbeitern per Fragebogen ausgewählt.

Im Rahmen der 'Wisconsin Sleep Cohort Study' wurde von Young et al. (1993) eine deutlich höhere Prävalenz ermittelt. Sie untersuchten 602 Personen zwischen 30 und 60 Jahren aus einem Kollektiv von ca. 3800 staatlichen Angestellten in Wisconsin. Darunter waren 352 Männer, von denen 225 mit Hilfe von Fragebögen als „, regelmäßige Schnarcher“ klassifiziert wurden und 250 Frauen, von denen 130 „Schnarcher“ waren. Die Prävalenz von SRBD, definiert als Apnoe-Hypopnoe-Index (AHI) von mindestens 5, wurde während einer Nacht im Schlaflabor ermittelt. Die Prävalenz von SRBD betrug 24\% bei Männern und 9\% bei Frauen. Bei 4\% der Männer und 2\% der Frauen wurde ein Schlafapnoe-Syndrom (SAS) diagnostiziert (AHI > 5 und exzessive Tagesmüdigkeit).

Neuere Studien zeigten ähnliche Ergebnisse. So ermittelten Bixler et al. (2001) aus 741 Männern (Durchschnittsalter 48,2 Jahre) und 1000 Frauen (48,8 Jahre) zufällig ausgewählter Bürger der allgemeinen Bevölkerung des südlichen Pennsylvania eine Prävalenz der Schlafapnoe von 3,9\% bei Männern und 1,2\% bei Frauen.

Diagnostisches Kriterium war ein im Schlaflabor polysomnographisch bestimmter AHI >10 mit begleitender Tagesmüdigkeit.

Kim et al. (2004) ermittelten an einem Kollektiv von 457 koreanischen Bürgern zwischen 40 und 69 Jahren eine Prävalenz von SRBD (AHI >5) von $27 \%$ bei Männern und $16 \%$ bei Frauen. Die Prävalenz eines OSAS (AHI >5 plus exzessive Tagesmüdigkeit) betrug 4,5\% bei Männern und 3,2\% bei Frauen. Die genannten Ergebnisse wurden polysomnographisch ermittelt. Bei 137 Probanden wurde die PSG zu Hause durchgeführt. Die restlichen 320 Probanden verbrachten eine Nacht zur Messung im Schlaflabor. 


\subsubsection{Komorbidität bei SAS}

Als Folge des Schlafapnoe-Syndroms bzw. SRBD leiden die Patienten unter einer erhöhten Mortalität und Morbidität (He et al., 1988). Findley et al. (1992) fanden eine erhöhte Inzidenz von Verkehrsunfällen bei Patienten mit einem Schlafapnoe-Syndrom (SAS). Dem SAS wird eine Rolle bei der Pathogenese von kardiovaskulären Erkrankungen zugeschrieben. Die Ergebnisse retrospektiver Studien legen nahe, dass Schlafapnoe ein Risikofaktor für kardiovaskuläre Morbidität und Mortalität ist (He et al., 1988).

\subsubsection{Arterieller Hypertonus}

So wurde von einer Prävalenz der OSA von etwa $30 \%$ bis $40 \%$ bei Patienten mit bekanntem arteriellen Hypertonus berichtet (Kales et al., 1984 und Fletcher et al., 1985). Es besteht eine Koinzidenz von OSA mit einer erhöhten Variabilität des Blutdrucks und mit einem Verlust des zirkadianen Blutdruckprofiles (Davies et al., 2000). In großen prospektiven Studien wurde gezeigt, dass die OSA ein Risikofaktor für art. Hypertonus ist (Lavie P et al., 2000).

Die Sleep Heart Health Study ermittelte eine odds ratio (OR) von 1.37 (95\% confidence interval $[\mathrm{CI}]: 1,03-1,83 ; \mathrm{p}=0,005)$ für SRBD und Bluthochdruck. Das untersuchte Kollektiv bestand aus 6132 Patienten (47,2\% Männer, 46,7\% >= 65 Jahre alt) aus laufenden Kohortenstudien über kardiovaskuläre oder respiratorische Erkrankungen. Bei diesen 6132 Patienten wurde eine Heim-PSG durchgeführt. Es ergab sich ein mittlerer AHI von 8,8/h. Der AHI korrelierte positiv mit dem systolischen Blutdruck der Patienten (Regressionskoeffizient $\beta=1,21)$ (Nieto et al., 2000).

Die Wisconsin Sleep Cohort Study verfolgte bei 709 Patienten über 4 Jahre den Zusammenhang von OSA und der Entwicklung des Hypertonus. Dabei zeigte sich ein AHI > 15 als unabhängiger Faktor für die Entwicklung eines art. Hypertonus. Die OR betrug 2.89 (95\% CI: 1,46-5,64) (Peppard et al., 2000).

In den Guidelines des Joint National Committee on Prevention, Detection, Evaluation, and Treatment of High Blood Pressure wird OSA als einer der wichtigsten Ursachen für art. Hypertonus angeführt (Chobanian et al., 2003). 


\subsubsection{Cerebrovaskuläre Insuffizienz}

Mehrere Studien konnten zeigen, dass Patienten mit cerebrovaskulären Ereignissen häufig an OSA leiden. Das Schnarchen, eines der Hauptsymptome der OSA, ist ein Risikofaktor für cerebrovaskuläre Insulte (Palomaki, 1991). $71 \%$ bzw. 62,5 \% der Apoplex - und TIA Patienten haben nach Dyken et al. (1996) bzw. Bassetti et Aldrich (1999) eine OSA. Auch in der Sleep Heart Health Study konnte ein signifikanter Zusammenhang von OSA und cerebrovaskulären Ereignissen gezeigt werden (Shahar et al., 2001). Wessendorf et al. untersuchten 147 Patienten im Alter zwischen 18 und 80 Jahren (95 Männer, 52 Frauen; Durchschnittsalter 61,3 Jahre) innerhalb von 3 Monaten nach dem Auftreten eines ersten Apoplexes mittels Polysomnographie. Die polysomnographisch ermittelte Prävalenz der SRBD lag bei $61,2 \%$ für einen RDI $>=5 / \mathrm{h}$, bei $43,5 \%$ für einen RDI $>10 / \mathrm{h}$, bei $32 \%$ für einen RDI > 15/h und bei 21,8\% für einen RDI > 20/h. Der Großteil der Störungen war obstruktiver Natur. Anhand dieser Ergebnisse wird OSA als Risikofaktor für cerebrovaskuläre Insulte angesehen.

Dieser Zusammenhang wird teilweise durch Störungen der Autoregulation des cerebralen Blutflusses bei OSA-Patienten erklärt (Balfors et Franklin, 1994). Außerdem konnte ein erhöhter intrakranieller Druck (Jennum et Borgesen, 1989) und ein reduzierter cerebraler Blutfluss (Fischer et al., 1992 und Netzer et al., 1998) bei Schlafapnoe-Patienten nachgewiesen werden. Anderen Untersuchungen zufolge besteht bei OSA-Patienten eine erhöhte Koagulabilität (Wessendorf et al., 2000).

\subsubsection{Koronare Herzkrankheit (KHK)}

OSA ist unabhängiger Risikofaktor für KHK und myokardiale ischämische Ereignisse (Hung et al., 1990 und Peker et al., 1999).

Andreas et al. (1996) konnten bei 50\% von Patienten mit KHK eine OSA feststellen. Die Sleep Heart Health Study zeigte ein signifikant höheres Risiko für das Auftreten einer KHK bei Patienten mit OSA (Shahar et al., 2001).

Bei Patienten mit OSA kann es zu nächtlichen ST-Senkungen im EKG kommen, die mit der Schwere des OSAS korrelieren (Koehler et al., 1991 und Hanly et al., 1993). Häufig kommt es bei Patienten, die an KHK und OSA erkrankt sind, zu apnoe-assoziierten Ischämien. Diese können über die Aktivierung des ZNS zu Arousals führen, die zur Störung der Schlafstruktur beitragen (Schäfer et al., 1997). 
Patienten mit OSA und KHK haben eine deutlich höhere kardiovaskuläre Mortalität (Peker et al., 2000) und eine entsprechend schlechtere Langzeitprognose (Mooe et al., 2001) als KHKPatienten ohne OSA.

Risikofaktoren der KHK, wie Hyperlipidämie (Koskenvuo et al., 1987) und Diabetes mellitus (Reichmuth et al., 2005), treten ebenso gehäuft bei Patienten mit OSA auf.

\subsubsection{Herzinsuffizienz}

Laut Sleep Heart Health Study ist OSA ein stärkerer Risikofaktor für die Ausbildung einer Herzinsuffizienz (OR 2,38, $95 \%$ Konfidenzintervall: 1,22-4,62), als für andere kardiovaskuläre Erkrankungen (OR Apoplex: 1,58, 95 \% KI 1,02-2,46; OR KHK: 1,27, 95\% KI 0,99-1,62) (Shahar et al., 2001). Andere Studien zeigen eine hohe Prävalenz von SRBD bei Patienten mit Herzinsuffizienz. Javaheri et al. diagnostizierten bei $51 \%$ der über zwei Nächte polysomnographisch untersuchten 81 männlichen Herzinsuffizienz-Patienten SRBD (AHI > 15/h). 40\% der Patienten hatten eine ZSA und 11\% eine OSA.

Nach anderen Studien ist die OSA mit linksventrikulärer Hypertrophie, systolischer Dysfunktion (Laaban et al., 2002) und diastolischer Dysfunktion (Fung et al., 2002) assoziiert. Die Funktionsstörungen werden durch repetitive Phasen, in denen ein negativer intrathorakaler Druck herrscht, beeinflusst. Es resultiert ein Anstieg der linksventrikulären Nachlast (Buda et al., 1979) und eine verminderte linksventrikuläre Relaxation (Virolainen et al., 1995). Die Apnoe-bedingte Hypoxie behindert die Funktionsfähigkeit der Myozyten (Cargill et al., 1995). Hypoxie und Arousals haben eine Aktivierung des sympathischen Nervensystems zur Folge. Dies wiederum kann zu einem Anstieg der Herzfrequenz und einer peripheren Vasokonstriktion führen, wodurch die Nachlast des ohnehin schon belasteten linken Ventrikels noch weiter erhöht wird (Somers et al., 1995).

\subsubsection{Arrhythmien}

Kardiale Arrhythmien sind mit OSA assoziiert (Guilleminault at al., 1983). Dabei sind verschiedene Formen von Brady- und Tachyarrhythmien zu beobachten, die in ihrem Ausmaß von der Schwere der OSA und den daraus resultierenden Hypoxien abhängig sind (Shepard et al., 1985). Vor allem Vorhofflimmern und ventrikuläre Arrhythmien sind häufig (Javaheri et al., 1998). Bradyarrhythmien werden als Folge der vagalen Antwort auf die Apnoen angesehen (Zwillich et al., 1982). Tachyarrhythmien werden hingegen von der erhöhten Aktivität des Sympathikus verursacht (Gami et al., 2004). 


\subsubsection{Pulmonalarterielle Hypertonie}

Als Folge der Hypoxie während einer Apnoephase steigt der pulmonal-arterielle ('pulmonary arterial pressure', PAP) Blutdruck (Bonsignore et al., 1994). Auf dem Boden des rezidivierenden akuten pulmonalen Hypertonus (PH) kann es zur Ausbildung eines chronischen PH kommen. Dies lassen die Studien von Sajkov et al. (1994), Sanner et al. (1997) und Bady et al. (2000) annehmen.

So untersuchten Sajkov et al. 27 Patienten mit OSAS (26 Männer, 1 Frau, mittleres Alter 49 Jahre). Bei 11 dieser Patienten (41\%) wurde ein pulmonaler Hypertonus diagnostiziert.

Sanner et al. betrachteten ein Patientenkollektiv von 92 OSAS-Patienten (81 Männer, 11 Frauen, mittleres Alter 53,1 Jahre). Die Diagnose eines pulmonalen Hypertonus (pulmonalarterieller Druck > 20mmHg) wurde bei 18 der 92 OSAS-Patienten (20\%) gestellt.

Bady et al. untersuchten 44 Patienten mit einem Schlafapnoe-Syndrom. Ein pulmonaler Hypertonus konnte bei 12 Patienten (27\%) festgestellt werden.

\subsubsection{Pathophysiologie}

Die Pathophysiologie der OSA ist komplex und beruht auf einem multifaktoriellen Geschehen, welches zu einer Instabilität der oberen Atemwege führt (Deegan et McNicholas, 1995). Verschiedene Mechanismen und prädisponierende Faktoren sind an der Entstehung der Obstruktion der oberen Atemwege beteiligt. Folge sind bei partieller Obstruktion HypopnoePhasen und bei totaler Obstruktion Apnoe-Phasen. Diese Ereignisse verursachen Hypoxien und enden jeweils mit einer Weckreaktion (Arousal). Dies führt wiederum zu Störungen der Schlafarchitektur und den daraus resultierenden Symptomen, wie exzessive Tagesmüdigkeit (American Academy of Sleep Medicine Task Force, (AASM, 1999)).

$\mathrm{Zu}$ den allgemeinen prädisponierenden Faktoren gehört das Geschlecht. Männer haben eine größere Prävalenz für OSA als Frauen (Guilleminault et al., 1976). Dies ist durch einen signifikant höheren pharyngealen und supraglottischen Atemwegswiderstand der Männer erklärbar, der im Alter in Verbindung mit einer entsprechenden Gewichtszunahme weiter zunimmt (White et al., 1985).

Adipositas ist mit Schlafapnoe assoziiert (Walsh et al., 1972). Dieser Zusammenhang lässt sich durch eine vermehrte Fetteinlagerung in den Pharynxwänden erklären (Shelton et al., 
1993). Stradling und Crosby beschrieben 1991 zudem in einem Kollektiv von 1001 Männern mittleren Alters eine enge Korrelation des Nackenumfangs mit der Inzidenz von OSA.

Eine genetische Komponente in der Ätiologie der OSA wird aufgrund von Familienstudien ebenfalls diskutiert (El Bayadi et al., 1990).

Alkoholkonsum fördert die Frequenz und Dauer der obstruktiven Phasen durch Reduktion des Muskeltonus der oberen Atemwege und Unterdrückung der Arousalmechanismen (Issa et Sullivan, 1982). Einen ähnlich negativen Effekt auf den Muskeltonus in den oberen Atemwegen haben Diazepam (Bonora et al., 1985), Chloralhydrate (Hershenson et al., 1984) und Anästhetika (Hwang et al., 1983).

Weitere negative Faktoren auf die Stabilität und den Durchmesser der oberen Atemwege sind tonsilläre Hypertrophie (Orr et Martin, 1981), Choanalatresie, Mikrognathie und craniofaciale Dysostosen (Schafer, 1982). Des Weiteren beeinflussen Infiltrationen in Gewebe und Muskeln der oberen Atemwege die Funktion dieser Muskeln und reduzieren den Durchmesser der Atemwege. Dies kann durch ein Myxödem bei Hypothyreose (Orr et al., 1981), Akromegalie (Grunstein et al., 1991), neoplastische Prozesse (Zorick et al., 1980) und Muccopolysaccharidose (Perks et al., 1980) bedingt sein.

Eine Zunahme des Atemwegswiderstandes wird außerdem von einer Obstruktion im Bereich der Nase durch akute oder chronische Rhinitis (Rundcrantz, 1969), auch allergischer Genese (McNicholas et al., 1982), durch Polypen (Cole et Haight, 1984) oder Septumdeviationen (Lavie P et al., 1982) hervorgerufen. Ein langes, weiches Zäpfchen, ein besonders tief platziertes Zungenbein (Riley et al., 1983) und eine Retrognathie (Rivlin et al., 1984) sind beteiligt an der Entstehung einer obstruktiven Komponente.

Die häufig bevorzugte Rückenlage während des Schlafes führt durch Wirkung der Gravitationskräfte (Fouke et Strohl, 1987) zu einer Abnahme des pharyngealen Durchmessers und somit zu einer Zunahme des Atemwegswiderstandes (Anch et al., 1982). Bedingt durch Hypoxie und Hyperkapnie kommt es zu Koordinationsstörungen zwischen den bei der Atmung beteiligten Muskeln im oberen Atemtrakt und dem Diaphragma. Dies wird als möglicher prädisponierender Faktor für OSA angesehen (Weiner et al., 1982). Hinzu kommen Störungen in den Reflexmechanismen in den oberen Atemwegen (Chadwick et al., 1991). Dies kann zu einem Ungleichgewicht zwischen intrapharyngealem Druck und Funktion der dilatierenden Muskeln führen (Remmers et al., 1978). Eine Beteiligung von Defekten der zentralen respiratorischen Kontrolle wird ebenfalls diskutiert (McNicholas et al., 1984).

Am Ende einer obstruktiven Apnoephase steht ein Arousal. Die genauen Mechanismen der Entstehung dieser Weckreaktion sind noch unklar. Drucksensitive Mechanorezeptoren in den 
oberen Atemwegen (Basner et al., 1992), Hypoxämie-sensible Rezeptoren in den Carotiden (Bowes et al., 1981) und vor allem Hyperkapnie-sensible Rezeptoren in den oberen Atemwegen und der Medulla (Berthon-Jones et Sullivan, 1984) scheinen eine Rolle zu spielen.

Die Hypoxien, Hyperkapnien und Arousals führen zu einer Erhöhung der Aktivität des sympathischen Nervensystems (Somers et al., 1989 und Morgan et al., 1995). Die Folge ist eine stark vermehrte Ausschüttung von Catecholaminen, wie Noradrenalin und Adrenalin in der Nacht aber auch am Tage (Fletcher et al., 1987). Dies führt wiederum zu einer Erhöhung des Gefäßwiderstandes und des systemischen, sowie des pulmonalen Blutdrucks (Shepard, 1985). Somit gilt die Überaktivität des Sympathikus als ein entscheidender Faktor der kardiovaskulären Morbidität, beispielsweise bei der Pathogenese eines Hypertonus und kardialer Hypertrophie (Izzo, 1989). Zudem haben betroffene Patienten eine erhöhte Blutdruckvariabilität, eine im Durchschnitt erhöhte Herzfrequenz (HF) und eine herabgesetzte Variabilität der HF. Diese Veränderungen der kardiovaskulären Variabilität werden als Risiko für die Entstehung von kardiovaskulären Erkrankungen angesehen (Narkiewicz et al., 1998).

OSA, mit der damit verbundenen hohen Sympathikusaktivität und den rezidivierenden Hypoxien scheint eine unabhängige Rolle in der Pathogenese der Atherosklerose zu spielen (Drager et al., 2005). So wurden verschiedene Mechanismen untersucht, die bei OSAPatienten zu einer beschleunigten Entstehung von Atherosklerose und der damit verbundenen erhöhten Rate an vaskulären Erkrankungen und Komplikationen führen können. Eine wichtige Rolle in der Pathogenese einer endothelialen Dysfunktion spielen bei OSA erhöhte Werte von CRP (Shamsuzzaman et al., 2002) - einem Marker der systemischen Entzündung und somit wichtigen Faktor in der Ätiologie der Atherosklerose -, Interleukin 6 (IL-6) (Yokoe et al., 2003) und Homocystein (Lavie L et al., 2001).

Zudem wurden Abnormalitäten verschiedener Mediatoren beschrieben. Endothelin-1, eine stark vasokonstriktorisch wirkende Substanz die vom Endothel der Gefäße produziert wird, hat einen erhöhten Spiegel bei OSA (Phillips et al., 1999). Die typischen Hypoxien und anschließenden Reoxygenierungen bei OSA fördern die Bildung freier oxidativer Radikale, die oxidativen Stress auf die vaskulären Endothelien ausüben könnten. Dieser Mechanismus schädigt die Zellen und Gewebe und ist mit der Entstehung einer KHK assoziiert (Lavie L, 2003). Verschiedene Adhäsionsmoleküle, von denen ebenfalls erhöhte Werte bei OSA beschrieben wurden, sollen auch die Entstehung der Atherosklerose begünstigen. Dazu 
gehören das intercellular adhesion molecule 1, das vascular cell adhesion molecule 1 und Eselectin (Chin et al., 2000).

Störungen des Gerinnungsystems werden ebenfalls als mögliche Faktoren bei der Genese kardiovaskulärer Erkrankungen bei OSA angesehen. So sind die Serum-Fibrinogen-Spiegel und die Blutviskosität erhöht (Chin et al., 1996). Es kommt zudem zu einer verstärkten Thrombozytenaktivierung und -aggregation (Bokinsky et al., 1995). Dagegen ist die Fibrinolyseaktivität vermindert (Rangemark et al., 1995).

\subsubsection{Diagnostik bei SAS}

Die überwachte nächtliche Polysomnographie (PSG) im Schlaflabor gilt als Goldstandard in der Diagnostik der Schlafapnoe (Chesson et al., 1997).

Hinsichtlich der Kosten und des Nutzen erscheint allerdings folgende Diagnostik in vier Stufen sinnvoll (Gemeinsamer Bundesausschuss, 2004).

\section{Stufe:}

Eine gezielte Anamnese mit Schwerpunkt auf den typischen Symptomen und Risikofaktoren der Schlafapnoe. Unterstützt wird dies von einem möglichst standardisierten Fragebogen.

\section{Stufe:}

Es folgt eine körperliche Untersuchung mit Fokus auf Stoffwechselerkrankungen, HerzKreislauf-Erkrankungen, Erkrankungen des Atem- und Lungenapparates sowie neurologische oder psychische Störungen. Eine anatomische Atemwegsobstruktion muss ebenfalls ausgeschlossen werden.

\section{Stufe:}

Bei Verdacht auf ein SAS folgt ein Screening mit einem ambulanten System, welches in der Lage ist eine kontinuierliche Registrierung von Atemfluss, Sauerstoffsättigung des Blutes, Herzfrequenz, Schnarchgeräuschen und Körperlage während des Schlafes durchzuführen.

Sollte sich hierbei kein pathologischer RDI (Grenzwert je nach Gerät und Definition 5-10/h) ergeben, so kann auf eine weitere Diagnostik verzichtet werden. Dies gilt allerdings nur sofern keine Symptomatik vorliegt, die auf ein SAS hinweisen könnte. 


\section{Stufe:}

Bei positivem Befund in der 3.Stufe oder entsprechender klinischen Symptomatik folgt eine polysomnographische Untersuchung in einem Schlaflabor. Dort erfolgt eine Registrierung und Analyse von EEG, EOG (Elektrookulogramm), EMG (Elektromyogramm), oronasalem Atemfluss, Oberkörperexkursionen, Schlafposition, Schnarchgeräuschen, EKG und Blutsauerstoffsättigung.

Mit der PSG ist die Einschätzung des Schweregrades und die Typisierung der schlafbezogenen Atemstörung möglich. Im Falle einer OSA ist ein AHI zwischen 5 und 15/h gleichbedeutend mit einer milden Ausprägung, ein AHI von 15 bis 30/h mit einer mittelschweren und ein AHI von über 30/h mit einer schweren Ausprägung (AASM, 1999).

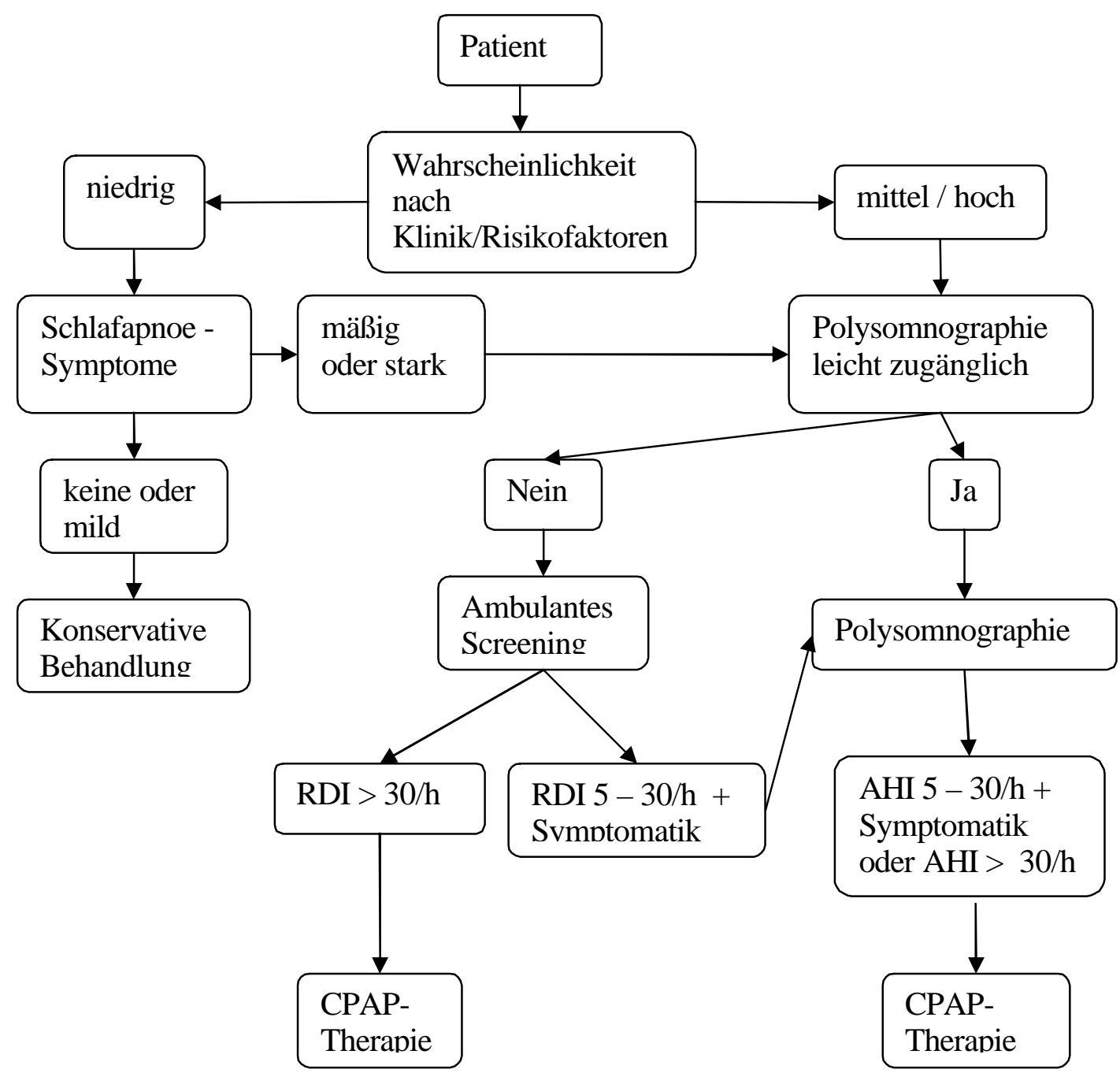

Abbildung 1: Flussdiagramm zur Diagnostik modifizert nach Flemons, 2002, S. 499 


\subsubsection{Therapie des SAS}

Wichtiger Bestandteil der Therapie sind allgemeine Maßnahmen. Dazu gehören Gewichtsreduktion bei Adipositas (Peppard et al., 2000), Schlafpositionstraining zur Vermeidung der Rückenlage (Oksenberg et al., 1997), Reduktion des abendlichen Alkohol (Taasan et al., 1981) und Benzodiazepinkonsums (Berry et al., 1995).

Ein möglicher Nutzen einer medikamentösen Therapie mit verschiedenen Substanzen (z.B. Antidepressiva, Antiendorphine, Theophylline) ist noch nicht abschließend gesichert. Ein Einsatz wird nicht empfohlen (Schäfer et al., 1996), da die unerwünschten Nebenwirkungen den therapeutischen Nutzen überwiegen (Wright et Dye, 1995).

Eine weitere Therapieoption stellen orale Schienen dar, die über Lageveränderungen der Weichteile und des Kiefers die Kollapsneigung in den oberen Atemwegen verringern sollen. Laut einem Bericht der American Sleep Disorders Association (ASDA, 1995) ist diese Therapieform aber nicht die erste Option, da Langzeitergebnisse fehlen. Gleiches gilt für die elektrische Stimulation muskulärer Anteile des Pharynx (Guilleminault et al., 1995).

Es stehen diverse chirurgische Therapieoptionen zu Verfügung. Mit einer Tracheotomie lässt sich eine OSA erfolgreich behandeln (Guilleminault et al., 1981), dies ist aber erst bei Versagen anderer nicht-operativer Methoden eine Option. Weitere chirurgische Möglichkeiten wie die Uvulopalatopharyngoplastik (UPPP) (Sher et al., 1996) und die LaserUvulopalatoplastik (LUPP) (Littner et al., 2000) werden wegen geringer Effizienz nicht empfohlen.

Die chirurgische Behandlung spezieller anatomischer Abnormalitäten wie Fehlbildungen im Kieferbereich (Riley et al., 1993) oder einer adenotonsillären Hypertrophie (Stradling et al., 1990) führt zur Besserung der Symptomatik.

Das Therapieverfahren der Wahl ist die kontinuierliche Überdruckbeatmung (Continuous Positive Airway Pressure, CPAP). Sie wird meist mittels einer Nasenmaske (nCPAP) durchgeführt und verhindert als "pneumatischer Keil" einen Kollaps der oberen Atemwege (Sullivan et al., 1981). Die Behandlung mit CPAP ist indiziert bei Patienten mit einem RDI > 30/h sowie bei Patienten mit einem RDI zwischen 5 und 30/h und durch die SRBD hervorgerufenen Beschwerden und/oder kardiovaskulären Erkrankungen (Loube et al., 1999). Die CPAP-Therapie führt zur Besserung der Tagesmüdigkeit, die Schlaffragmentation (Bennett et al., 1999) und die Unfallhäufigkeit nehmen ab (George, 2001). Die Lebensqualität steigt mit dem Abfall des AHI (Sanner et al., 2000). Eine effektive CPAP-Therapie führt zur Reduktion des Blutdrucks (Becker et al., 2003). Dies wird durch eine verminderte 
sympathische Aktivität (Somers et al., 1995) sowie eine erhöhte NO-Produktion (Schulz et al., 2000) erklärt.

Bei Patienten mit Herzinsuffizienz führt die Anwendung von CPAP zu einer verbesserten linksventrikulären Funktion (Kaneko et al., 2003) und u.a. zu einer Verringerung der linksventrikulären Nachlast (Bradley et al., 1992). CPAP reduziert die Zahl der nächtlichen Ereignisse von Angina pectoris und der myokardialen Ischämien (Franklin et al., 1995).

Kardiale Arrhythmien wie Bradykardien (Grimm et al., 2000), ventrikuläre und supraventrikuläre Tachykardien (Harbison et al., 2000) werden genauso von CPAP positiv beeinflusst, wie pulmonaler Hypertonus (Alchanatis et al., 2001). Unter CPAP -Therapie wird eine Normalisierung der erhöhten Infektparameter CRP und IL-6 (Yokoe et al., 2003) erreicht. Ebenso normalisieren sich die Thrombozytenaktivität und -aggregation (Bokinsky et al., 1995), sowie die Werte atherogener Substanzen wie Endothelin-1 (Phillips et al., 1999). Trotz der Effektivität der CPAP-Therapie brechen etwa 10\% der Patienten die Therapie ab (Collard et al., 1997).

\subsection{Terminale Niereninsuffizienz und chronische Nierenersatz-} therapie

Erstmals führte Kolff 1943 eine Hämodialyse am Menschen durch (Zit. bei Reinhardt et Krick, 1997). Die von Alwall entwickelte Trommelniere ermöglichte 1948 eine Ultrafiltration (Borger 1997). Die Entwicklung des Scribner - Shuntes (1960) führte zur Schaffung eines permanenten Gefäßzuganges, so dass sich im Laufe der sechziger Jahre die sogenannte „Nierenersatztherapie“ mit der Hämodialyse, der Hämofiltration und der Peritonealdialyse etablieren konnte. Die erste Nierentransplantation wurde durch Merrill und Murray im Jahr 1954 durchgeführt (Merril et al., 1956).

Nach Daten des QuaSi-Niere Jahresberichtes 2003/2004 ist ein stetiger Zuwachs der Patientenzahlen in chronischer Nierenersatztherapie zu verzeichnen. Während 1995 in Deutschland 41350 Patienten von der Dialyse abhängig waren, waren es im Jahr 200358579 Patienten (Frei et Schober-Halstenberg, 2004). 


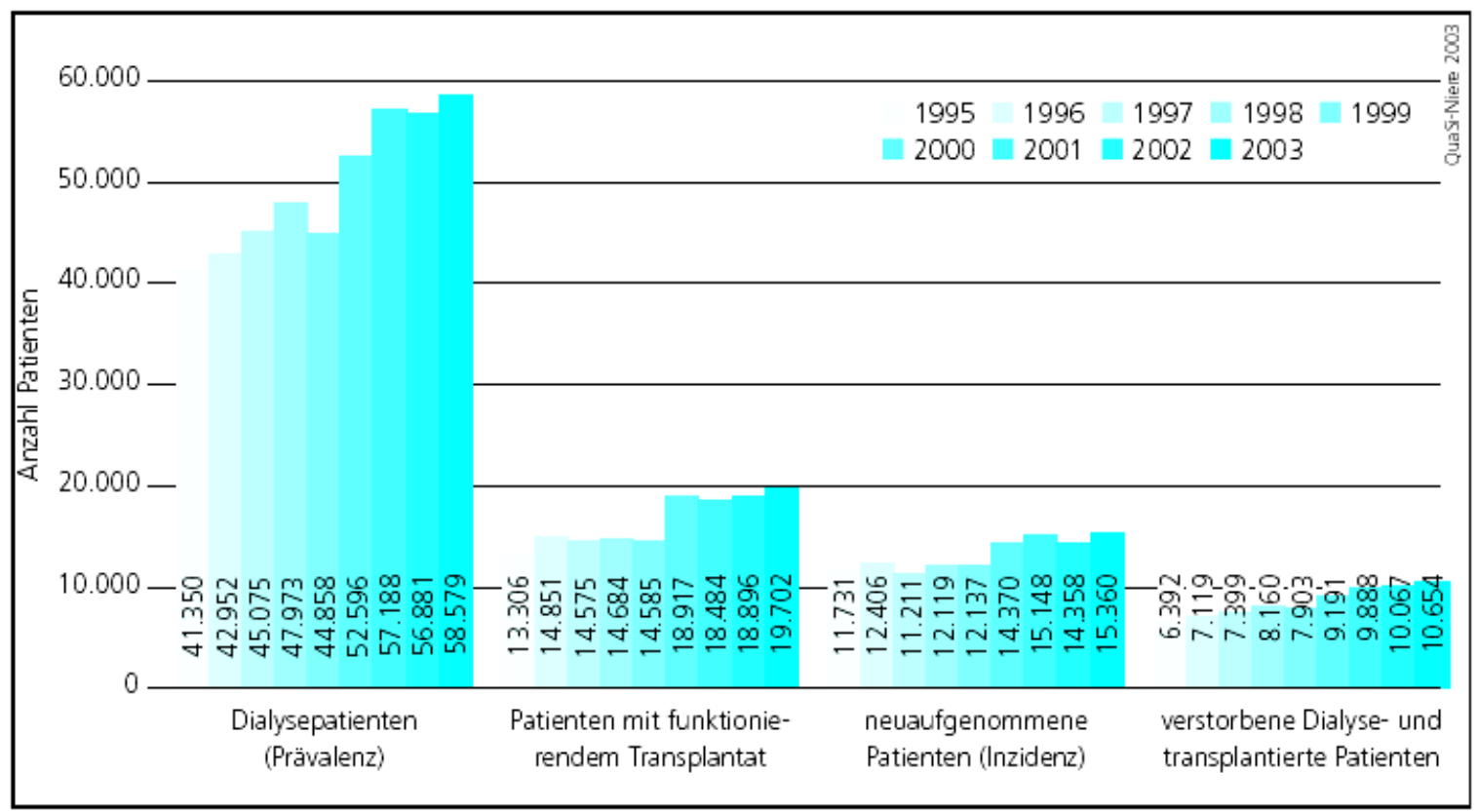

Abbildung 2: Jahresvergleich: Chronische Nierenersatztherapie in Deutschland 1995 - 2003 (nach Frei et Schober-Halstenberg, 2004, S.33)

Die zunehmende Inzidenz des Diabetes mellitus als dominierende zur terminalen Niereninsuffizienz führende Grunderkrankung gilt als Schrittmacher für diese Entwicklung. Weiterhin führen verbesserte Dialysetechniken, als auch eine effektivere Kontrolle und Therapie von Begleiterkrankungen zu einer verlängerten Lebenserwartung.

Die der terminalen Niereninsuffizienz zu Grunde liegenden Erkrankungen sind in Abbildung 3 dargestellt. 


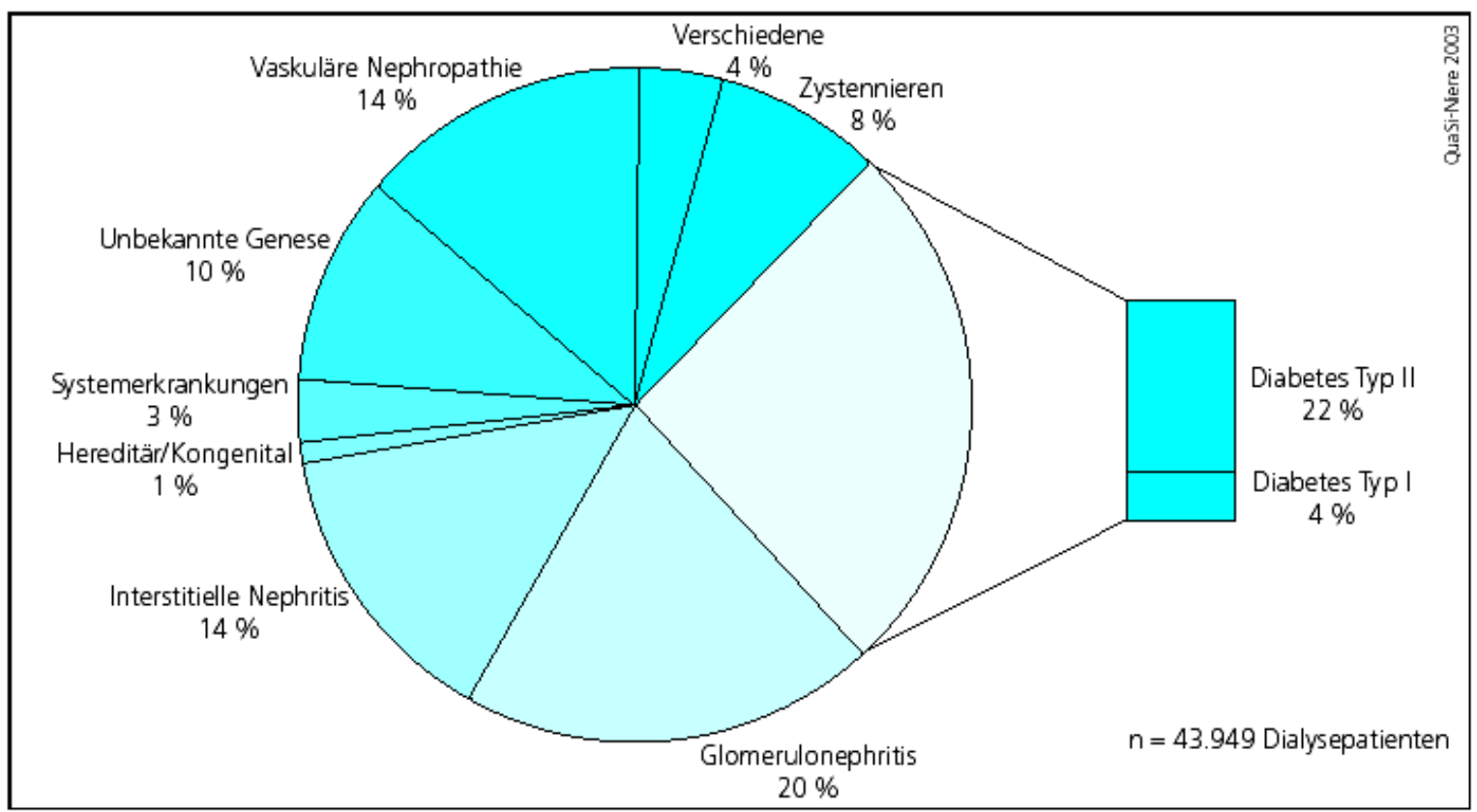

Abbildung 3: Diagnoseverteilung aller bei QuaSi-Niere erfassten lebenden Dialysepatienten in Nierenersatztherapie (Prävalenz) 2003 (nach Frei et Schober-Halstenberg, 2004, S.35)

Gemessen am Langzeitüberleben und der Morbidität stellt die Nierentransplantation die bestmögliche Therapieoption bei terminaler Niereninsuffizienz dar (Parsons et Harris, 1997).

Die 1-Jahres-Patienten- und Transplantatüberlebensrate beträgt 90 bis 95\%. Allerdings werden 10 bis $20 \%$ der transplantierten Patienten pro 5-Jahresintervall erneut dialysepflichtig (Frei et Schindler, 2000 b). Nach Angaben von Frei et Schober-Halstenberg im Namen der QuaSi-Niere GmbH wurden 20032516 Patienten nierentransplantiert. 9479 Patienten standen auf der Warteliste. Durch die begrenzte Anzahl der verfügbaren Spendernieren ist mit keiner wesentlichen Steigerung der Transplantationszahlen $\mathrm{zu}$ rechnen. Die chronische Dialysebehandlung stellt daher die zurzeit quantitativ wichtigste Behandlungsform der terminalen Niereninsuffizienz dar. Das wesentliche Ziel der Dialyseverfahren ist es, urämiebedingte Organschäden zu verhindern. Zielwerte stellen hierbei die Verlängerung der Überlebenszeit und Verbesserung der Lebensqualität dar (Frei et Schindler, 2000 a). 
Folgende Dialyseverfahren stehen zur Verfügung (Reinhardt et Krick, 1997):

- Hämodialyse: Hämodialyse wird bei ca. $86 \%$ aller Patienten angewandt. Hierbei findet der Stoffaustausch zwischen Blut und Dialysat per Diffusion niedermolekularer Substanzen über eine semipermeable Membran statt.

- Hämofiltration: Bei diesem seltener angewandten Verfahren erfolgt der Stofftransport per Konvektion, d.h. Plasmawasser wird mit den darin gelösten niedermolekularen Substanzen über eine semipermeable Mebran abgepresst. Das Entstehen eines Volumendefizits wird durch eine entsprechend gesteuerte Substitution einer Elektrolytlösung verhindert.

- Weiterhin kommen Kombinationsverfahren, die sog. Hämodiafiltration zum Einsatz.

Neben den extrakorporalen Verfahren wird die Peritonealdialyse angewandt, bei der das Peritoneum als Dialysemembran dient und so harnpflichtige Substanzen und Wasser mittels Diffusion, osmotischer Ultrafiltration und konvektivem Stofftransport eliminiert werden (Reinhardt et Krick, 1997; Lonnemann et al., 2000).

Trotz der in den letzten Jahren verbesserten Dialysetechniken und der deutlich gebesserten Transplantationsergebnisse, verstarben 200310654 der 58579 Dialysepatienten, darunter 339 Nierentransplantierte. Die Haupttodesursache sind kardiovaskuläre Erkrankungen (43\%). Es folgen infektionsbedingte Todesfälle mit 17,7\% (Frei et Schober-Halstenberg, 2004). Die Mortalitätsrate der Dialysepatienten liegt in den letzten Jahren bis zu 20-mal höher als in der Normalbevölkerung (Amann et Ritz, 1995; Mall, 2000). 


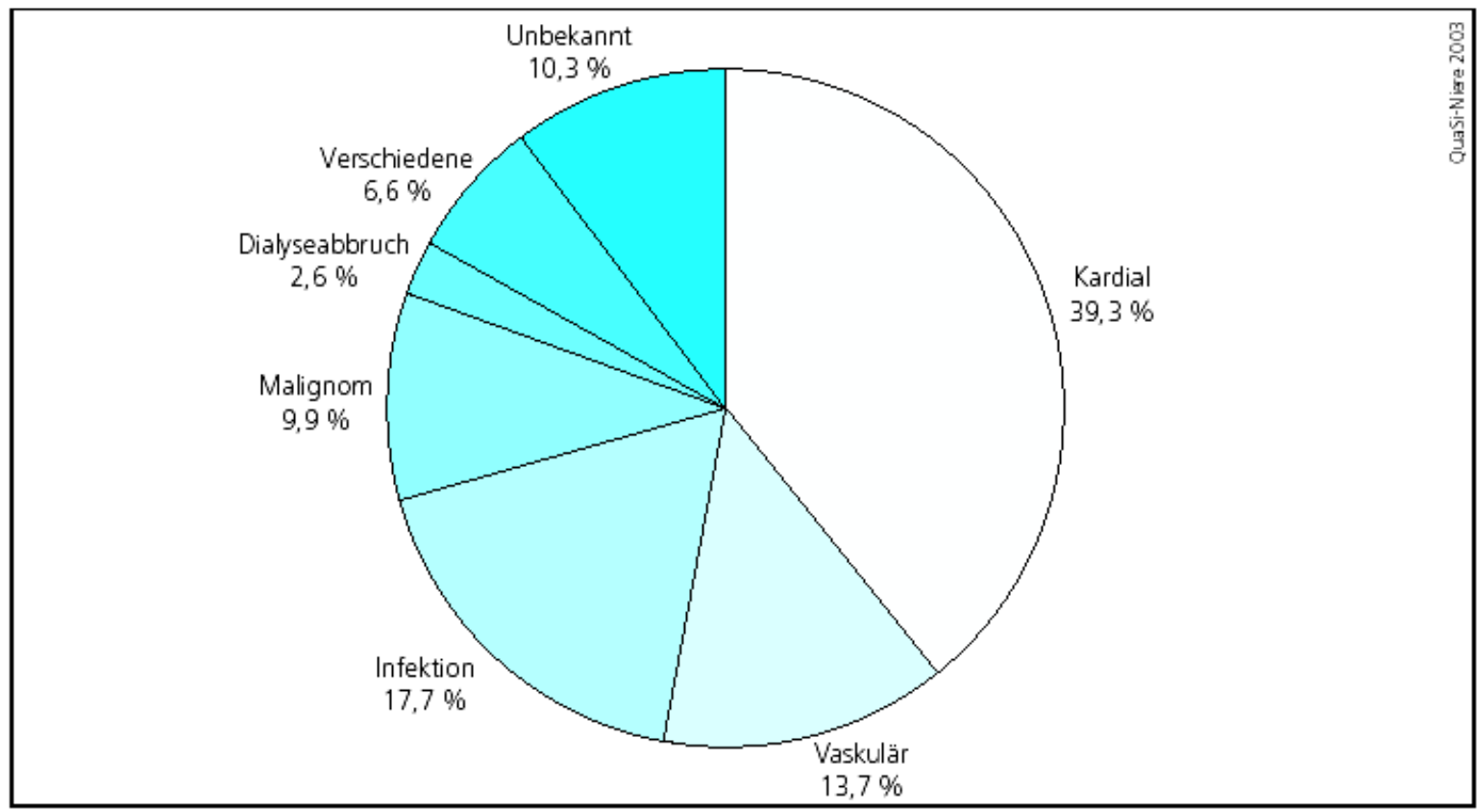

Abbildung4: Todesursachen der im Jahre 2003 verstorbenen Patienten (nach Frei et SchoberHalstenberg, 2004, S.38)

\subsection{Schlafbezogene Atemstörungen bei Dialysepatienten}

\subsubsection{Epidemiologie}

Bei Dialysepflichtigen Patienten bestehen häufig Schlafstörungen. Je nach Studie können derartige Störungen bei 48 bis 77\% der Patienten festgestellt werden (de Santo et al., 2005; Mucsi et al., 2004; de Barbieri et Zampieron, 2004). Neben Ein - und Durchschlafstörungen sowie dem Syndrom der unruhigen Beinen (Restless-legs Syndrom, RLS), wird häufig eine obstruktive Schlafapnoe (OSA) beobachtet.

In folgenden Studien wurde die Prävalenz der OSA mit Hilfe von Fragebögen bei Dialysepflichtigen Patienten abgeschätzt: Walker et al. 1995 ermittelten mit Hilfe eines Fragebogens bei sieben von 54 Dialysepatienten (13\%) Symptome einer OSA. BurmannUrbanek et al. erhoben Daten an 69 Dialysepatienten. Die Auswertung der Fragebögen ergab bei 46 Patienten (67\%) Hinweise auf relevante Schlafstörungen. Die Angabe von nächtlichem Schnarchen, nächtlichen Atempausen und Tagesmüdigkeit wurde bei 13 Patienten (19\% des Gesamtkollektivs) als Anzeichen für eine Schlafapnoe gedeutet. 
In anderen Studien wurde die Prävalenz der OSA mittels ambulanter Screeningverfahren ermittelt:

Kuhlmann et al. führten bei 55 von 84 niereninsuffizienten Patienten aus 2 Dialysezentren in der Nacht nach der Dialyse ein ambulantes Screening durch. Die Auswertung ergab bei 30,9\% dieser Patienten einen AHI >5/h. Insgesamt erfüllten $16,4 \%$ der Patienten die Diagnosekriterien eines OSAS, da sie zusätzlich eine ausgeprägte Tagesmüdigkeit aufwiesen. Hallet et al. zeichneten bei 11 HD-(Hämodialyse) Patienten (5 Männer, 6 Frauen; Durchschnittsalter: 58 Jahre) bzw. 10 CAPD-(Continuous Ambulatory Peritoneal Dialysis) Patienten (6 Männer, 4 Frauen; Durchschnittsalter: 63 Jahre) über eine Nacht den nasalen Atemfluss und die Sauerstoffsättigung auf. 73\% (8 von 11) bzw. 70\% (7 von 10) der Patienten zeigten eine signifikante Schlafapnoe definiert als einen AHI $>15 / \mathrm{h}$.

Die Bestimmung der Prävalenz einer Schlafapnoe durch eine Polysomnographie (PSG) gilt als die genaueste diagnostische Methode (Goldstandard).

Hallet et al. untersuchten 9 HD und 6 CAPD Patienten. 89\% (HD) bzw. 67\% (CAPD) der Patienten zeigten eine klinisch relevante OSA.

Ähnliche Ergebnisse ermittelten Wadhwa et Mendelson (1992). Sie diagnostizierten bei 8 von 15 (12 Männer, 3 Frauen) (53\%) HD-Patienten und bei 9 von 15 (11 Männer, 4 Frauen) (60\%) CAPD-Patienten eine Schlafapnoe.

Schwer einzuschätzen ist die klinische Bedeutung der Schlafbezogenen Atemstörungen (Sleep-related breathing Disorder, SRBD) bei Patienten mit terminaler Niereninsuffizienz.

Inwieweit die Ergebnisse der 3 beschriebenen Diagnosemöglichkeiten (Fragebögen, ambulantes Screening, PSG) miteinander vergleichbar sind, ist unklar und anzuzweifeln. Die diagnostische Sicherheit der einzelnen Tests ist bei Dialysepatienten bisher nicht untersucht.

So überschneiden sich die Symptome der Schlafapnoe teilweise mit denen der terminalen Niereninsuffizienz, der Anämie bei Dialysepatienten oder des Restless-Legs-Syndroms (RLS). Dies lässt vermuten, dass die Spezifität der üblichen Fragebögen der Schlafapnoediagnostik bei Dialysepatienten deutlich reduziert ist. 


\subsubsection{Spezielle Pathogenese und Therapie}

Die Ursache der hohen Inzidenz der Schlafstörungen bei Patienten mit ESRD (End-StageRenal-Disease) ist noch nicht vollständig geklärt. Die Niereninsuffizienz selbst scheint jedoch zur Ausbildung von SRBD zu prädisponieren, da eine Besserung der Schlafstörungen durch Dialyse (Fein et al., 1987) und Nierentransplantation (Aukley et al., 1999) beschrieben wurden.

Pro-inflammatorische Zytokine sind möglicherweise an der Pathogenese der Schlafapnoe bei Patienten mit ESRD beteiligt (Zoccali et al., 2002 a). Schlafapnoe-Patienten weisen erhöhte Werte für die Zytokine tumor necrosis factor-alpha (TNF-a) und interleukin-6 (IL-6) (Vgontzas et al., 2000) auf. Erhöhte Zytokinwerte werden zudem bei Dialysepatienten gefunden, wobei die vermehrte Induktion der leukozytären Zytokinproduktion den Dialysemembranen zugeschrieben wird (Rousseau et al., 2000).

Urämische Toxine scheinen mitverantwortlich für die Schlafstörungen bei Dialysepatienten zu sein. Strub et al. vermuteten 1982 einen Zusammenhang der Schlafstörungen mit der Retention von „, Mittel-Molekülen“.

Nach Fein et al. sind starke Reduktionen des Tonus der Atemmuskulatur und Koordinationsstörungen zwischen Diaphragma und Muskulatur der oberen Atemwege beteiligt an der Pathogenese von OSA bei ESRD-Patienten. Dies ist bedingt durch negative Einflüsse von „Urämietoxinen“ oder hormonellem Ungleichgewicht auf das zentrale Nervensystem. Metabolische Abnormalitäten könnten ebenso mitverantwortlich für die Entstehung einer Schlafapnoe sein (Langevin et al., 1993), wobei ein Zusammenhang zwischen einer metabolischen Azidose und der Entstehung einer OSA (Ingbar et Gee., 1985) nicht bestätigt werden konnte (Kimmel et al., 1989).

Störungen des Kalziumstoffwechsels, mit Folge einer Hyperkalzämie können zu Schlafstörungen führen (Virga et al., 2000). Zudem wird eine direkte toxische Wirkung des Parathormons bei niereninsuffizienten Patienten auf das zentrale Nervensystem mit Einfluß auf die Pathogenese von Schlafstörungen diskutiert (Fraser et Arieff, 1988).

Urämie-bedingte Störungen im Aminosäurenhaushalt, wie niedrige Taurinwerte (Furst, 1989) scheinen ebenfalls eine Rolle zu spielen.

Eine urämische Enzephalopathie (Hughes, 1980) und die periphere Neuropathie (Culebras, 2001) sind ebenfalls mit Schlafstörungen assoziiert. So scheint eine autonome Neuropathie in Verbindung mit einem Diabetes Mellitus mit Störungen der Atemkontrolle während des Schlafes einherzugehen (Neumann et al., 1995). Zudem ist die respiratorische Antwort auf 
Hyperkapnie bei Dialysepatienten mit autonomer Neuropathie signifikant reduziert (Auinger et al., 1995).

Eine Anämie scheint ein Faktor bei der Entstehung einer Schlafapnoe zu sein, da eine Therapie mit rekombinantem humanem Erythropoetin eine Besserung der Symptomatik zeigte (Benz et al., 1999). Des Weiteren wurde die Hypothese aufgestellt, dass die Akkumulation von endogenen Opioiden über eine Destabilisierung der Atmung zu einer OSA führt (McQueen, 1983 und Zoccali et al., 1987). Untersuchungen zur Bestätigung dieser Hypothese fehlen allerdings bislang.

Störungen des Wasserhaushalts bei ESRD im Sinne einer pharyngealen Ödembildung könnten zu einer Obstruktion der oberen Atemwege führen und somit zur Pathogenese einer OSA beitragen (Fein et al., 1987).

Während der Dialyse bestehende Episoden mit Erhöhungen der Körpertemperatur und damit verbundene Veränderungen im zirkadianen Körpertemperaturmuster (Parker et al., 2000) sind weitere mögliche Erklärungen für Schlafstörungen und eine ausgeprägte Tagesmüdigkeit bei Dialysepatienten.

Allgemeine Begleitsymptome der Urämie, wie Juckreiz, Muskelkrämpfe und Knochenschmerzen, könnten ebenfalls eine Rolle bei der Genese von Schlafstörungen spielen (Parfrey et al., 1988).

Darüber hinaus kommen psychogene und psychoreaktive Faktoren (Livesley, 1981), auch im Rahmen depressiver Krankheitsbilder (Craven et al., 1987) bei Patienten mit ESRD als Ursache für Schlafstörungen in Betracht.

Die Relevanz der OSA bei ESRD-Patienten ergibt sich aus dem Zusammenhang zwischen der Assoziation von SRBD und kardiovaskulären Erkrankungen (KVE) (Newman et al., 2001), da beide Erkrankungen bei Dialysepatienten sehr häufig sind. Wie o.g. sind KVE die Haupttodesursache bei Dialysepatienten (Frei et Schober-Halstenberg, 2004). Die hohe kardiovaskuläre Morbidität und Mortalität bei ESRD-Patienten lässt sich nicht ausschließlich durch die traditionellen Risikofaktoren wie HTN, Dyslipidämie und Diabetes Mellitus erklären. Andere Risikofaktoren, wie OSA spielen möglicherweise ebenfalls eine Rolle (Zoccali et al., 2003).

So sind die bei der OSA auftretenden nächtlichen Hypoxämien für Störungen im autonomen Nervensystem, mit erhöhter Sympathikusaktivität und Schwankungen in der parasympathischen Antwort verantwortlich (Smith et al., 1998). Kardiovaskuläre autonome Dysfunktionen werden unabhängig davon bei Dialysepatienten beobachtet (Robinson et Carr, 2002). Bei Dialysepatienten mit OSA werden daher die Hypoxämien für die autonome 
Dysfunktion und folglich für die Förderung kardiovaskulärer Ereignisse verantwortlich gemacht (Zoccali et al., 2001 b). Folge der Hypoxämien sind ein erhöhter nächtlicher arterieller Blutdruck (Zoccali et al. 1998), eine linksventrikuläre konzentrische Hypertrophie (Zoccali et al., 2001 a) und ein schlechteres kardiovaskuläres Outcome bei Dialysepatienten (Zoccali et al., 2002 b).

Dazu beitragen könnten auch die gestörte Insulinsensitivität (Strohl et al., 1994) und Störungen in der endothelialen Funktion (Carlson et al., 1996) bei SA-Patienten. Diese Faktoren bedingen ein erhöhtes kardiovaskuläres Risiko und spielen auch bei Morbidität und Mortalität von ESRD-Patienten eine wichtige Rolle (Charlesworth et al., 2005 und Mysliwiec et al., 2004).

Schlafapnoe ist durch erfolgreiche Nierentransplantation reversibel. Dies deutet darauf hin, dass Schlafapnoe eine Folge der Niereninsuffizienz ist (Langevin et al., 1993 und Aukley et al., 1999). Durch intensive Hämodialyse ist Schlafapnoe ebenfalls reversibel (Fein et al., 1987), wobei besonders die tägliche nächtliche Hämodialyse effektiv zu sein scheint (Hanly et al., 2003).

Die CPAP-Therapie gilt auch bei ESRD-Patienten als erfolgreiche Therapie der Schlafapnoe (Pressman et al., 1993). 


\section{Fragestellung}

Schlafbezogene Atemstörungen (SRBD) zeigen insbesondere bei chronisch niereninsuffizienten Patienten, die eine Nierenersatztherapie erhalten, eine im Vergleich zur Normalbevölkerung ausgesprochen hohe Prävalenz. Die Diagnose einer schlafbezogenen Atemstörung wird gegenwärtig jedoch nur bei wenigen Patienten gestellt.

Das Ziel der vorliegenden Untersuchung war es, die Prävalenz der SRBD, insbesondere der Schlafapnoe, der Dialyse-Patienten der Station 1011 (chronische Dialyse) des Universitätsklinikums Göttingen zu bestimmen.

Es sollte ferner geklärt werden, ob sich die Symptomatik und das Risikoprofil von Dialysepatienten von dem anderer Populationen unterscheidet. Der Einfluss der Atemstörungen auf die kardiovaskuläre Morbitidät und Mortalität sowie der Häufigkeit des Auftretens von Infektionskrankheiten sollte untersucht werden.

Mit den erhaltenen Daten sollte eine Power-Analyse zur Planung einer größeren Studie zur Klärung des Zusammenhangs von SRBD bei Dailysepatienten und kardiovaskulärer Morbidität und Mortalität durchgeführt werden.

Zusammengefasst standen folgende Fragen im Mittelpunkt der durchgeführten Untersuchung:

- Wie hoch ist die Prävalenz des Schlafapnoe-Syndroms im Patientenkollektiv der Dialysestation 1011 des Universitätsklinikums Göttingen?

- Gelten die üblichen Risikofaktoren des Schlafapnoe - Syndroms auch für chronisch niereninsuffiziente Patienten mit Nierenersatztherapie?

- Können die bislang bei „Normalpatienten“ angewandten diagnostischen Mittel (v.a. standardisierte Fragebögen) zur (Früh)-Erkennung eines Schlafapnoe-Syndroms auch bei Dialysepatienten sinnvoll eingesetzt werden?

- Gibt es einen nachweisbaren Zusammenhang zwischen der Schlafapnoe und der kardiovaskulären Morbidität und Mortalität bei Dialysepatienten?

- Gibt es einen nachweisbaren Zusammenhang zwischen der Infektanfälligkeit und der Prävalenz der Schlafapnoe bei Dialysepatienten? 
2 Fragestellung

- Durchführung einer Power-Analyse (Power 0,8, Signifikanz $p<0,05$ ) zur Schätzung der notwendigen Patientenzahl zur Erhöhung der Inzidenz kardiovaskulärer Ereignisse um $10 \%$. 


\section{Patienten, Material und Methoden}

Die chronisch niereninsuffizienten Patienten der Station 1011 wurden mit Hilfe eines ambulanten Schlafapnoe-Screeninggerätes (Somno-Check effort der FirmaWeinmann) auf das Vorhandensein eines Schlafapnoe - Syndroms untersucht.

Vor Durchführung der Untersuchung erfolgte eine Fragebogen-basierte Evaluation bezüglich typischer Symptome von SRBD.

Ebenso erfolgte eine retrospektive Auswertung von Labor und klinischen Daten über einen Zeitraum von bis zu zwei Jahren vor Untersuchung. Dabei standen vor allem Daten zu kardiovaskulären Folge- und Begleiterkrankungen, sowie zur Häufigkeit durchgemachter Infektionen im Mittelpunkt des Interesses.

\subsection{Ein- und Ausschlusskriterien}

Alle 51 Patienten der Station 1011 des Universitätsklinikums Göttingen die folgende Einschlusskriterien erfüllten, wurden um ihre Einwilligung zur Teilnahme an der Studie gebeten:

- Bei dem Patienten liegt eine terminale Niereninsuffizienz vor, die mit einem extrakorporalen Nierenersatzverfahren oder Peritonealdialyse behandelt wird.

- Eine Nierenersatztherapie wurde bei Einschluss länger als 6 Monate durchgeführt.

Wenn bei den eingeschlossenen Patienten mindestens eines der folgenden Kriterien zutraf, wurde ein ambulantes Screening durchgeführt:

- Beantwortung wenigstens einer der Fragen auf dem „Schlafapnoe-Fragebogen" mit ,ja“ (s. Anlage 1)

- $\quad$ Epworth-Sleepiness-Scale (ESS) $>8$ (s.u.)

- $\quad$ BMI $>25 \mathrm{~kg} / \mathrm{m}^{2}$ (nach WHO-Definition als Maß für Übergewicht) oder Änderung des Körpergewichtes in den letzten 6 Monaten vor Einwilligung um mehr als $10 \%$

- Vorliegen eines besonderen kardiovaskulären Risikos. Im Einzelnen:

○ Hypercholesterinämie, LDL - Cholesterinerhöhung, HDL Cholesterinerniedrigung 
○ Arterielle Hypertonie

○ Bekannte atherosklerotische Erkrankungen (KHK, CVI, pAVK), auch bei bisher asymptomatischem Verlauf

- Vorliegen einer links- oder rechtsventrikulären Hypertrophie in der Echokardiographie.

Ausschlusskriterien für die Durchführung einer ambulanten Screening Untersuchung:

- Vorliegen einer infektiösen Erkrankung oder Besiedlung mit einem Problemkeim (z.B. Hepatitis C, MRSA)

- Fehlende Einwilligung in die Untersuchung

- Schwangerschaft.

Die Laborwerte und klinischen Daten zur kardiovaskulären Morbidität wurden der Krankenakte entnommen und in eine Access-Datenbank übertragen. Die Daten wurden für einen retrospektiven Beobachtungszeitraum von 2 Jahren vor Referenzdatum evaluiert.

Das Referenzdatum ist der Tag an dem das Schlafapnoe-Screening durchgeführt wurde. Patienten, die von einer ambulanten Screening Untersuchung ausgeschlossen waren, wurden mittels Fragebogen evaluiert. Die Laborwerte und klinischen Daten wurden entsprechend den tatsächlich untersuchten Patienten in die Datenbank übernommen. Dieses Vorgehen hatte den Zweck den Effekt eines Einschluss-Bias einzuschätzen.

\subsection{Fragebogen (siehe Anlage 1)}

Die Fragen wurden den Patienten vorgelesen und bei Bedarf erklärt.

\section{1. ,Allgemeinbefinden“}

Die Bewertung des Allgemeinbefindens auf einer Skala von 1-10 wurde erfragt. Wobei „1“ gleichbedeutend mit ,sehr gut“ und „10“ gleichbedeutend mit ,sehr schlecht“ war. 


\section{2. ,Epworth-Sleepiness-Scale“ (ESS)}

Die ESS beinhaltet Beschreibungen von 8 Situationen, die den Patienten täglich begegnen. Von den Patienten sollte eingeschätzt werden, mit welcher Wahrscheinlichkeit sie auf einer Skala von „,0“ (,würde niemals einnicken“) bis ,,3“ (,würde mit großer Wahrscheinlichkeit einnicken“) in der jeweiligen Situation einschlafen würden.

Es hat sich gezeigt, dass die Ergebnisse der ESS signifikant mit dem RDI korrelieren und ein ESS-Score größer 8 ein Indikator für eine signifikante subjektive Müdigkeit ist (Johns, 1991). Der ESS ist eine geeignete Methode zur Einschätzung der Tagesmüdigkeit (Johns, 1992) und ist in der Lage primäre Schnarcher von OSA-Patienten zu unterscheiden (Johns, 1993).

\section{3. ,Schlafapnoe-Fragen“6}

Typische Symptome einer Schlafapnoe sollten evaluiert werden. Dazu wurde die jeweilige Prävalenz von Tagesmüdigkeit, morgendlicher Unausgeschlafenheit, Schlafstörungen, nächtlichem Aufschrecken, Schnarchen und von Atempausen während des Schlafes erfragt. Außerdem wurde der Konsum von Schlafmedikamenten ermittelt.

\subsection{Durchführung des ambulanten Schlafapnoe-Screenings}

Das ambulante Schlafapnoe-Screening wurde mit dem Screening-Gerät „Somno-Check effort“ (Firma Weinmann, CE 0197) (siehe Abbildung 7) durchgeführt.

Das „Somno-Check effort“ Screening-Gerät wurde in Untersuchungen erprobt und aufgrund seiner Robustheit, der einfachen Handhabung und trotzdem komplexer Sensorik, sowie dem stark reduzierten Aufwand und den damit verbundenen geringen Kosten im Vergleich zu den konventionellen diagnostischen Verfahren empfohlen (Schöller et Kotterba, 2001). Das Gerät gilt als anerkannt und verlässlich zur Diagnosestellung einer OSA mit einer Sensitivität von 0,83 und einer Spezifität von 0,95 (Ficker et al., 2001).

„Somno-Check effort" registriert den Atemfluss und Schnarchgeräusche der Patienten mit Hilfe eines T-förmigen Kombi-Sensors, der das Atemflusssignal über den Temperaturunterschied zwischen Umgebungs- und Atemluft erfasst. Dazu besitzt dieser zwei spezielle Sensoren (Thermistoren) unterhalb der Nasenlöcher und einen weiteren Sensor zur Erfassung des oralen Atemflusses. Zusätzlich registriert ein Mikrofon Schnarchgeräusche.

Des Weiteren verfügt das Screening-Gerät über ein Pulsoxymeter zur Registrierung der Blutsauerstoffsättigung und der Pulsfrequenz. 
Ein in das Gehäuse des Gerätes integrierter Sensor registriert die Körperlage des Patienten während des Schlafes und das Gurtsystem des „Somno-Check effort“ beinhaltet spezielle Sensoren (Piezosensoren), die die Atembewegungen von Thorax und Abdomen (,Efforts“) messen. Zudem registrieren die Piezosensoren Muskelanspannungen, Krampf- und Rüttelbewegungen, sowie gegenläufige Bewegungen von Oberbauch und Zwerchfell. Diese Ereignisse sind Anzeichen für eine Behinderung der Atmung.

Den am Screening teilnehmenden Patienten wurde am Tag der Dialyse die Funktionsweise des Screening-Gerätes ausführlich erklärt.

Zudem wurde das Anlegen des Gerätes demonstriert und eine für den jeweiligen Patienten individuelle Anpassung vorgenommen.

Am selben Abend (also am Tag der Dialyse) wurde das Gerät von den Patienten selbstständig angelegt und bis zum folgenden Morgen getragen. Zum nächsten Dialysetermin wurde das Screening-Gerät wieder mitgebracht.

Das Screening-Gerät wurde zur Analyse an einen PC angeschlossen und die Daten vom integrierten Datenlogger anschließend auf den PC übertragen und mit Hilfe der zum Screening-Gerät gehörigen Software der Firma Weinmann visualisiert und automatisch analysiert. Dabei ist das System in der Lage, Apnoe- und Hypopnoephasen und obstruktive sowie zentrale Apnoen voneinander $\mathrm{zu}$ unterscheiden. Zusammenfassend werden die wichtigsten Analyseergebnisse automatisch in Form einer Befundübersicht angezeigt.

Als Kriterium für eine Apnoe galt eine Dauer von mindestens 10 Sekunden. Die Kriterien der Hypopnoe waren eine maximale Atemamplitude von 50\% und eine Mindestdauer von 10 Sekunden. Ein Blutsauerstoffsättigungsabfall wurde ab 4\% als solcher gewertet.

Ein RDI > 10/h wurde innerhalb der Studie als Kriterium für das Vorliegen einer Schlafapnoe angewandt.

Die Auswertung wurde in Zusammenarbeit mit der Abteilung Kardiologie/Pulmonologie des UKG durchgeführt. 


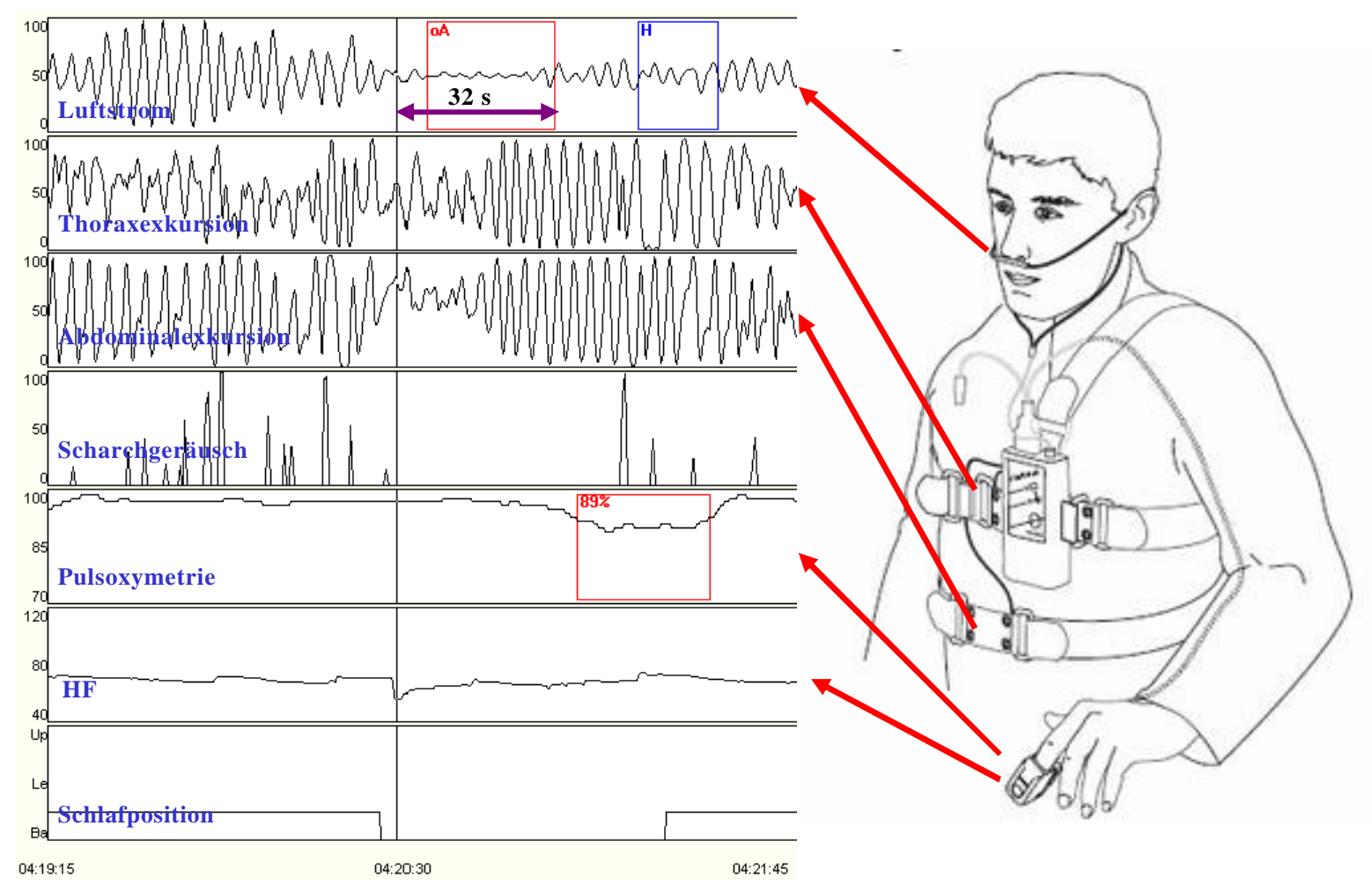

Abbildung 5: SOMNOcheck effort

\subsection{Retrospektive Daten}

Den Patientenakten der Station 1011 wurden die routinemäßig erfassten Laborwerte des Zentrallabors des Universitätsklinikums Göttingen entnommen und die errechneten Mittelwerte in die Datenbank eingetragen.

Zusätzlich wurden den Patientenakten die biometrischen Daten, die relevanten kardiovaskulären Diagnosen und Ereignisse, die Infektereignisse und die relevanten Dialysedaten entnommen.

Zudem wurde die aktuelle Medikation mit Einfluss auf die kardiovaskuläre Morbidität erfasst und in der Datenbank archiviert.

Die Datenbank wurde mit Hilfe des Programms Microsoft Access 2000 erstellt. 


\subsection{Auswertung der Daten/Statistische Verfahren}

Alle statistischen Analysen wurden mittels Microsoft Excel und Minitab 14 durchgeführt. Kontinuierliche Variablen wurden nach Prüfung auf Normalverteilung auf einen Unterschied zwischen Vergleichswerten mit dem Student`s-t-Test bzw. bei fehlender Normalverteilung mit dem Mann-Whitney-U-Test untersucht. Die deskriptive Statistik für kontinuierliche Variablen wurde als Mittelwert mit Standardabweichung angegeben. Zur Untersuchung von Unterschieden zwischen kategorialen Variablen diente bei der geringen Fallzahl der Fisher`s exact-Test.

In allen Fällen wurden zweiseitige p-Werte herangezogen. Als statistisch signifikant galt ein alpha-Fehler kleiner als 0,05.

Es wurde keine Anpassung des p-Wertes aufgrund von multiplen Tests vorgenommen. Alle Ergebnisse der Korrelationen zeigen nur Tendenzen an. 


\section{Ergebnisse}

\subsection{Patientenkollektiv}

Von den 51 Patienten der Station 1011 des Universitätsklinikums Göttingen wurden 9 Patienten aufgrund von bestehenden MRSA - Infektionen oder chronischen Hepatitis B/ C Infektionen aus hygienischen Gründen vom Schlafapnoe-Screening ausgeschlossen. 3 weiteren Patienten war es nicht möglich das Screening-Gerät selbstständig korrekt anzubringen, zudem stand ihnen zu Hause keine Hilfe seitens einer weiteren Person zur Verfügung. Diese Patienten konnten somit ebenfalls kein Screening erhalten. Ebenso ein weiterer Patient, der vor dem Untersuchungstermin verstarb.

Von den nicht ausgeschlossenen Patienten willigten 21 ein, an einem ambulanten Schlafapnoe - Screening teilzunehmen.

Von den ausgeschlossenen Patienten und den Patienten, die ein Screening abgelehnt hatten, willigten 23 ein an der Evaluierung per Fragebogen teilzunehmen. Zudem genehmigten sie die Übernahme der Laborwerte und klinischen Daten in die Datenbank.

Zusammenfassend konnten aus der Gruppe der 51 Patienten der Station 1011, 21 mit dem Schlafapnoe-Screening-Gerät „Somno - Check effort“ über die Dauer von einer Nacht beobachtet werden. 
Das am Schlafapnoe - Screening teilnehmende Patientenkollektiv ist folgendermaßen charakterisiert

\begin{tabular}{|l|l|l|}
\hline Patienten $\mathrm{n}=21$ & \multicolumn{1}{|c|}{$\mathrm{x}$} & \multicolumn{1}{c|}{$\mathrm{SD}$} \\
\hline Alter ( Jahre) & 68,8 & 15,2 \\
\hline Dialysealter(Jahre) & 3,87 & 3,66 \\
\hline Dialysezeit(Std./Wo.) & 14,02 & 1,65 \\
\hline Gewicht $(\mathrm{kg})$ & 70,81 & 10,38 \\
\hline Größe $(\mathrm{cm})$ & 167,5 & 6,91 \\
\hline BMI $\left(\mathrm{kg} / \mathrm{m}^{2}\right)$ & 25,28 & 3,87 \\
\hline CRP (mg/dl) & 11,28 & 12,45 \\
\hline Albumin $(\mathrm{g} / \mathrm{dl})$ & 3,895 & 0,2 \\
\hline Triglyceride (mg/dl) & 163,67 & 57,22 \\
\hline Cholesterin (mg/dl) & 157 & 30,8 \\
\hline Phosphat (mmol/l) & 1,66 & 0,37 \\
\hline Ca (mmol/l) & 2,38 & 0,17 \\
\hline P*Ca (mmol/l) & 48,62 & 10,19 \\
\hline Hb (mg/dl) & 11,67 & 0,86 \\
\hline mHN AD (mg/dl) & 65,44 & 12,26 \\
\hline mHN Diff. (mg/dl) & 44,37 & 8,36 \\
\hline RRsyst. AD (mmHg) & 138,76 & 17,54 \\
\hline RRsyst. ED (mmHg) & 125,19 & 17,97 \\
\hline RR Diff. (mmHg) & 13,5 & 14,01 \\
\hline mGR (ml) & 6942 & 2475 \\
\hline Diurese (ml) & 512,38 & 646,5 \\
\hline & & \\
\hline
\end{tabular}

Tabelle 2: Charakteristika des Patientenkollektivs $(\mathrm{x}=$ arithmetischer Mittelwert, $\mathrm{SD}=$ Standardabweichung, $\mathrm{P} * \mathrm{Ca}=$ Phosphat - Calcium - Produkt, $\mathbf{m H N}$ AD = mittlerer Harnstoff-N Wert vor Dialyse, $\mathbf{m H N}$ Diff. = mittlere Harnstoff-N Reduktion während der Dialyse, $\mathrm{RRsyst}$. $\mathrm{AD}=$ sytolischer art. Blutdruck am Beginn der Dialyse, RRsyst. ED = systolischer art. Blutdruck am Ende der Dialyse, mGR = Gewichtsreduktion während der Dialyse/Woche).

Von den 21 Patienten an denen ein Screening durchgeführt wurde, waren 14 männlich $(66,7 \%)$ und 7 weiblich (33,3\%). Das Alter dieser Patienten lag zwischen 31 und 91 Jahren mit einem Mittelwert von 68,8 Jahren $(\mathrm{s}=15,2)$.

Die Dauer der chronischen Dialysepflichtigkeit bis zum Referenzdatum betrug im Mittel 3,9 Jahre $(\mathrm{s}=3,66)$.

Die durchschnittliche Dialysedauer lag bei 14,02 Stunden pro Woche (s = 1,65), das Körpergewicht lag zwischen $49 \mathrm{~kg}$ und $89 \mathrm{~kg}$ mit einem Mittelwert von 70,81 kg ( In Verbindung mit der Körpergröße, die im Mittel bei 167,5cm ( $\mathrm{s}=6,91)$ lag wurde zudem der Body-Mass-Index (BMI) ermittelt. 
BMI $=$ Gewicht $(\mathrm{kg})$ : Körpergröße zum Quadrat $\left(\mathrm{m}^{2}\right)$.

Der mittlere BMI des Patientenkollektivs betrug 25,28 kg/m² $(\mathrm{s}=3,87)$. Wertet man einen BMI größer als $25 \mathrm{~kg} / \mathrm{m}^{2}$ als Übergewichtig, fanden sich 10 übergewichtige Patienten. Dies entspricht 47,6\% des Kollektivs der gescreenten Patienten.

Neben dem Übergewicht zeigten sich folgende Begleiterkrankungen als weitere kardiovaskuläre Risikofaktoren, sowie folgende Geschlechterverteilung des Kollektivs:

\begin{tabular}{|l|l|l|}
\hline & Anzahl $\mathrm{n}$ & Prozent \% \\
\hline Männlich & 14 & 66,7 \\
\hline Weiblich & 7 & 33,3 \\
\hline
\end{tabular}

Tabelle 3: Geschlechterverteilung

In 20 Fällen lag ein arterieller Hypertonus (HTN) vor (95,2\%). 7 Patienten (33,3\%) waren Diabetiker (DM) und bei 5 Patienten $(23,8 \%)$ war eine Hyperlipidämie (HLP) diagnostiziert worden.

Bei 10 Patienten (47,6\%) waren eine oder mehrere kardiovaskuläre Erkrankungen (KVE) diagnostiziert worden. Dies war bei $7(33,3 \%)$ eine KHK, bei $3(14,3 \%)$ eine cerebrovaskuläre Insuffizienz (CVI) und bei keinem Patienten eine pAVK. 
Kardiovaskuläre Erkrankungen und Risikofaktoren

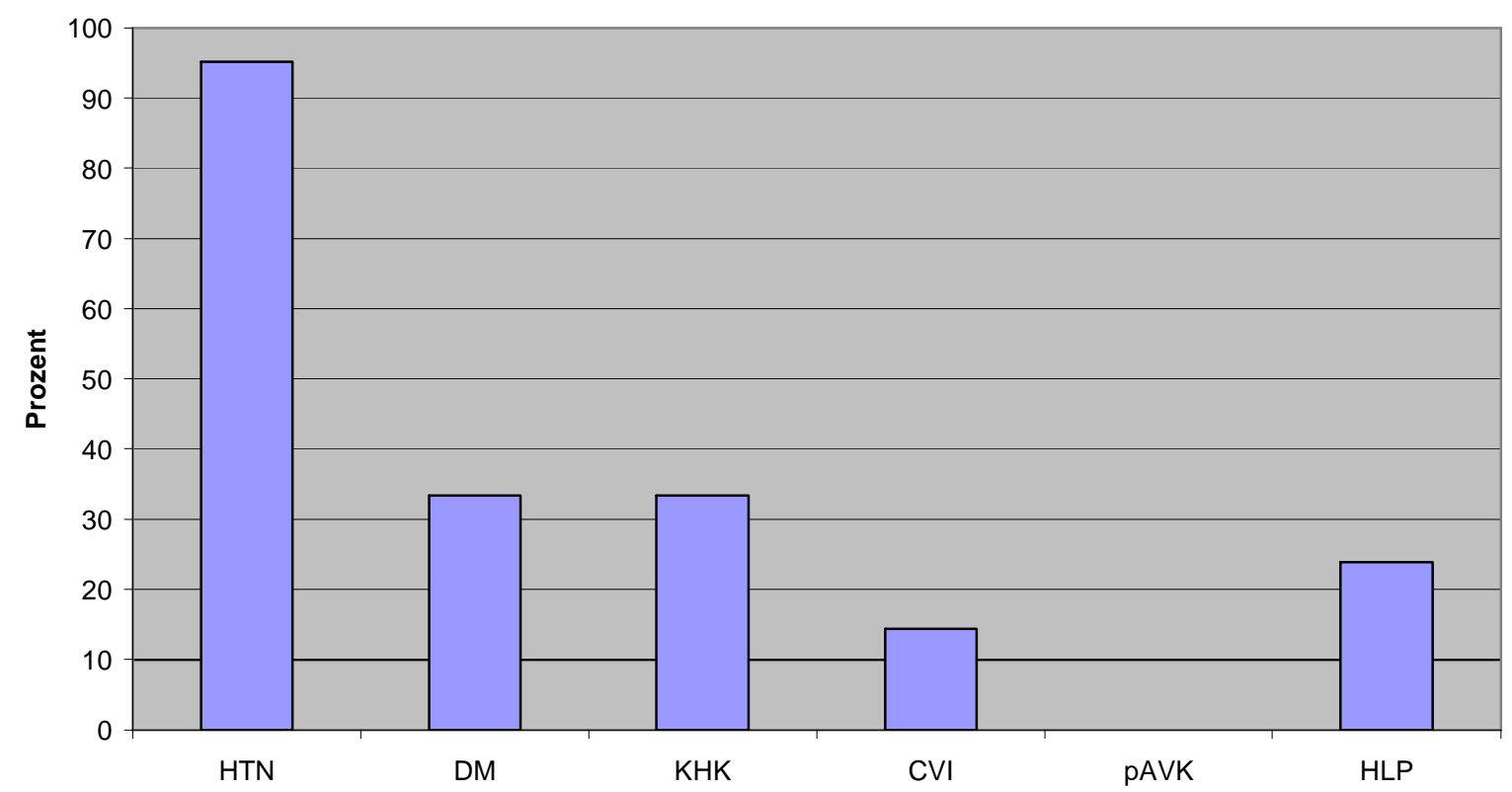

Abbildung 6: Kardiovaskuläre Erkrankungen und Risikofaktoren (HTN = arterieller Hypertonus, DM = Diabetes mellitus, KHK = Koronare Herzerkrankung, CVI = Cerebrovaskuläre Insuffizienz, pAVK = periphere Arterielle Verschlusskrankheit, HLP = Hyperlipidämie).

Die Ätiologie der terminalen Niereninsuffizienz stellt sich wie folgt dar:

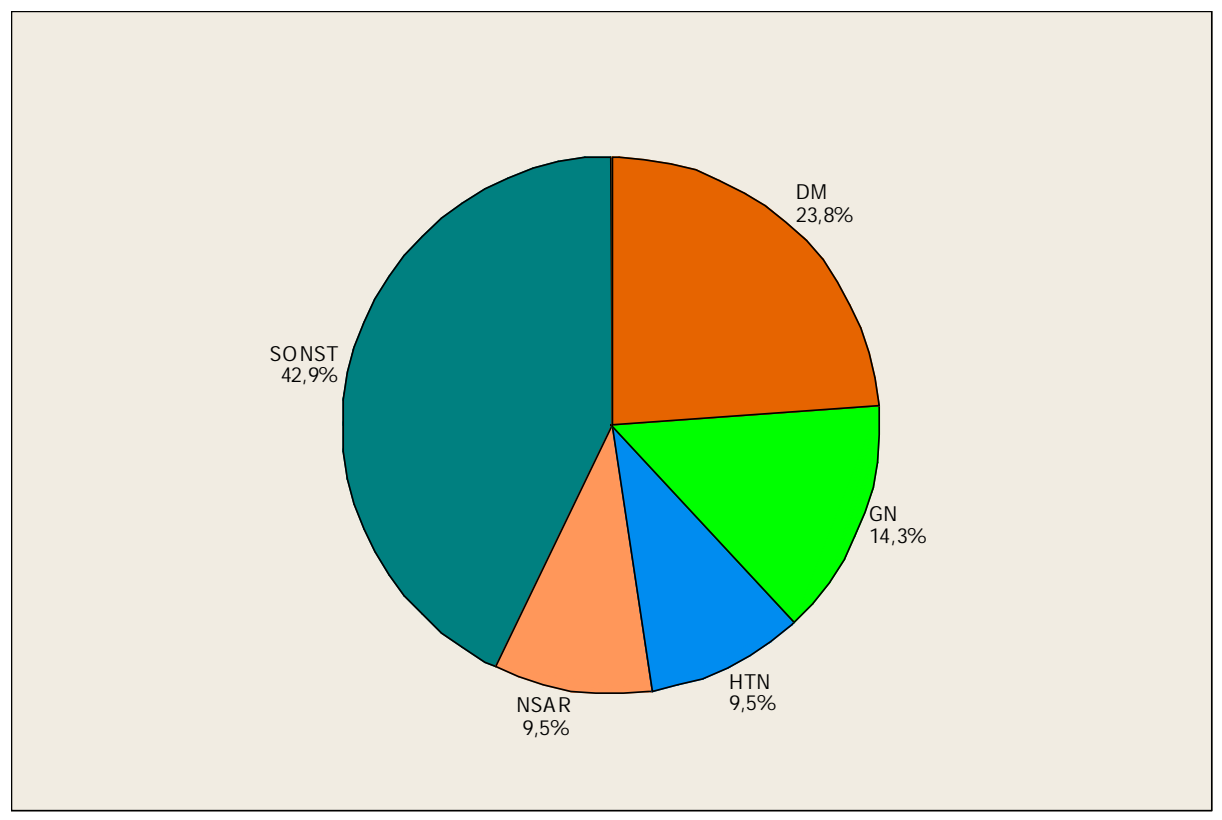

Abbildung 7: Ursachen der chronischen Niereninsuffizienz (DM = Diabetes mellitus, HTN = arterieller Hypertonus, NSAR = langfristige Einnahme nicht steroidaler Anti-Rheumatika, GN = Glomerulonephritiden, SONST = sonstige oder nicht eruierbare Ursachen). 
Zum Referenzdatum erfolgte eine medikamentöse Therapie der kardiovaskulären Risikofaktoren mit folgenden Stoffgruppen:

\begin{tabular}{|l|l|l|}
\hline Medikamentengruppe & Anzahl $\mathrm{n}$ & Prozent \% \\
\hline $\begin{array}{l}\text { Antihypertensiva } \\
\text { (insgesamt ) }\end{array}$ & 18 & 85,7 \\
\hline ACE-Hemmer & 1 & 4,8 \\
\hline AT-1 Antagonisten & 6 & 28,6 \\
\hline B-Blocker & 11 & 52,4 \\
\hline Ca- Antagonisten & 8 & 38,1 \\
\hline a-Blocker & 2 & 9,5 \\
\hline $\begin{array}{l}\text { Zentralwirksame } \\
\text { Antihypertensiva }\end{array}$ & 6 & 28,6 \\
\hline Diuretika & 10 & 47,6 \\
\hline Andere Antihypertensiva & 5 & 23,8 \\
\hline CSE-Hemmer & 9 & 42,8 \\
\hline Antikoagulanzien & 1 & 4,8 \\
\hline $\begin{array}{l}\text { Thrombozyten- } \\
\text { aggreationshemmer }\end{array}$ & 14 & 66,7 \\
\hline
\end{tabular}

Tabelle 4: Medikation bei Referenzdatum

\subsection{Auswertung des Fragebogens}

\begin{tabular}{|l|c|c|}
\hline & \multicolumn{1}{|c|}{$\mathrm{x}$} & $\mathrm{SD}$ \\
\hline Allgemeinbefinden & 3,86 & 1,98 \\
\hline ESS-Score & 5,14 & 3,6 \\
\hline
\end{tabular}

Tabelle 5: Ergebnisse des Fragebogens ( $x=$ arithmetischer Mittelwert, $\mathrm{SD}=$ Standardabweichung)

Die Selbsteinschätzung ihres Allgemeinbefindens auf einer Skala von 1 bis 10 ( 1 = sehr gut; 10 = sehr schlecht) durch die Patienten erbrachte Werte zwischen 1 und 7 mit einem Mittelwert von $\mathrm{x}=3,86(\mathrm{~s}=1,98)$.

Die Epworth Sleepiness Scale (ESS) der später gescreenten Patienten ergab Werte zwischen 0 und 14 bei einem Mittelwert von 5,14 (s=3,6). 4 Patienten (19\%) hatten einen ESS-Score > 8 als Indikator für eine signifikante subjektive Müdigkeit.

Die Auswertung des symptomspezifischen Fragebogens zeigte folgende Ergebnisse:

$6(28,6 \%)$ der 21 Patienten des Kollektivs gaben an, regelmäßig schlaffördernde Substanzen einzunehmen. 9 Patienten (42,9\%) bejahten eine bestehende Tagesmüdigkeit. Morgendliche Unausgeschlafenheit hatten 12 Patienten $(57,1 \%)$ und 3 Patienten $(14,3 \%)$ berichteten von 
nächtlichem Aufschrecken. Die Frage nach Schlafstörungen wurde von 13 Patienten $(61,9 \%)$ positiv beantwortet. 11 Patienten $(52,4 \%)$ gaben an, zu schnarchen. Bei 2 Patienten $(9,5 \%)$ waren nächtliche Atempausen bekannt.

Schlafapnoe - Fragen

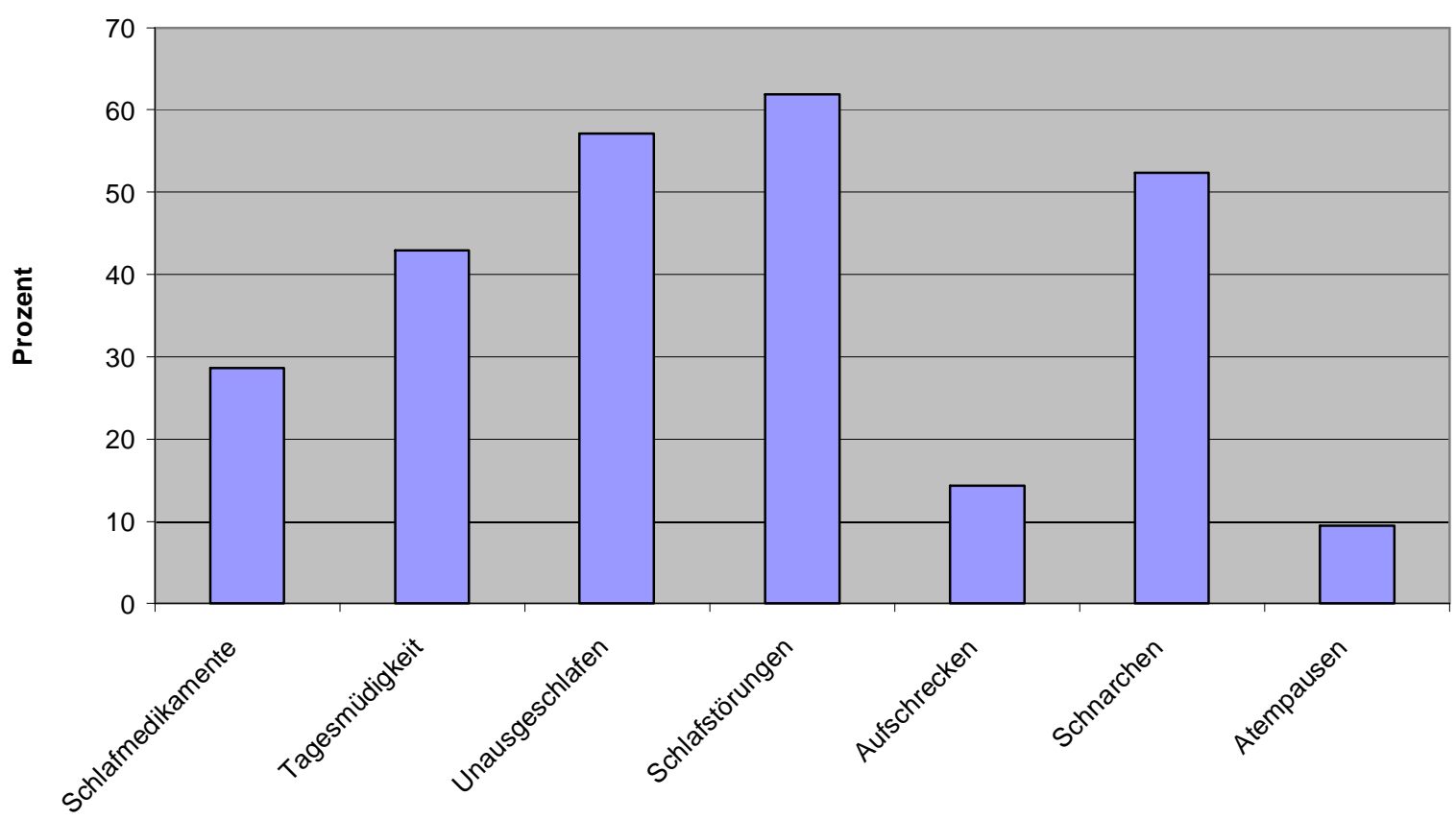

Abbildung 8: Ergebnisse des symptomspezifischen Fragebogens

\subsection{Ergebnisse des Schlafapnoe-Screenings}

\begin{tabular}{|l|l|l|}
\hline & \multicolumn{1}{|c|}{$\mathrm{x}$} & $\mathrm{SD}$ \\
\hline Gesamt Apnoen/Hypopnoe & 60,57 & 59,31 \\
\hline oA & 34,68 & 34,82 \\
\hline gA & 7,43 & 17,16 \\
\hline zA & 2,57 & 4,38 \\
\hline Hypopnoen & 15 & 13,25 \\
\hline RDI & $10,52 / \mathrm{h}$ & 10,64 \\
\hline O2 - Sättigung & $94,33 \%$ & 1,88 \\
\hline Entsättigungsindex & 15,24 & 12,67 \\
\hline Gesamtzahl Entsättigungen & 82,95 & 68,78 \\
\hline
\end{tabular}

Tabelle 6: Ergebnisse des Schlafapnoe - Screenings $(x=$ arithmetischer Mittelwert, SD = Standardabweichung, oA = obstruktive Apnoen, gA = gemischte Apnoen, zA = zentrale Apnoen) 
Die Gesamtanzahl von Apnoen und Hypopnoen betrug im Mittel 60,57 (s = 59,31) pro Patient und Nacht. Diese teilten sich auf in 34,68 (s = 34,82) obstruktive Apnoen, 7,43 ( $\mathrm{s}=17,61)$ gemischte Apnoen, 2,57 (s = 4,38) zentrale Apnoen und 15 ( $\mathrm{s}=13,25)$ Hypopnoen.

Der RDI bewegte sich zwischen 0 und 41/h und lag im Mittel bei 10,52/h $(\mathrm{s}=10,64)$

Bei 12 der 21 gescreenten Patienten $(57,1 \%)$ lag der RDI bei 5/h oder höher. 9 Patienten $(42,9 \%)$ erfüllten unsere Kriterien einer Schlafapnoe mit einem RDI von 10/h oder höher.

Darunter waren 7 Männer (77,8\%) und 2 Frauen (22,2\%). Von diesen Patienten zeigten 4 einen RDI von 20/h oder höher. Einer dieser Patienten hatte mit einem RDI > 40/h einen ausgeprägten Befund.

Die mittlere Blutsauerstoffsättigung lag bei 94,33\% ( $\mathrm{s}=1,88$ ). Insgesamt wurden 82,95 (s = 68,78) Entsättigungen pro Patient und Nacht registriert und ein mittlerer Entsättigungsindex von $15,24(\mathrm{~s}=12,67)$ bestimmt. 


\subsection{Vergleich der Daten in den Untergruppen}

Bei Vergleich der Daten der Patienten mit einem RDI $<10 /$ h $\left(\right.$,RDI $\left.<10^{\star *}\right)$ mit denen der Patienten mit einem RDI >= 10/h (,RDI >= 10“), also mit einer Schlafapnoe, ergibt sich folgendes:

\begin{tabular}{|c|c|c|c|c|c|}
\hline \multirow[t]{2}{*}{ Anzahl } & \multicolumn{2}{|c|}{$\begin{array}{l}\text { RDI > }=10 \\
\mathrm{n}=9(42,9 \%)\end{array}$} & \multicolumn{2}{|c|}{$\begin{array}{l}\text { RDI < } 10 \\
n=12 \quad(57,1 \%)\end{array}$} & \multirow{2}{*}{$\frac{p-W e r t}{t-T e s t}$} \\
\hline & $\mathrm{x}$ & SD & $\mathrm{X}$ & SD & \\
\hline Alter ( Jahre) & 71,2 & 11,37 & 66,9 & 17,85 & 0,509 \\
\hline Dialysealter(Jahre) & 5,33 & 4,77 & 2,78 & 2,18 & 0,166 \\
\hline Dialysezeit(Std./Wo.) & 14,67 & 0,66 & 13,54 & 2,02 & 0,094 \\
\hline Gewicht (kg) & 67,33 & 10,12 & 73,42 & 10,2 & 0,192 \\
\hline Größe (cm) & 169,56 & 8,06 & 166,0 & 5,8 & 0,282 \\
\hline BMI $(\mathrm{kg} / \mathrm{m} * 2)$ & 24,52 & 2,93 & 25,85 & 4,5 & 0,424 \\
\hline CRP (mg/dl) & 11,81 & 15,24 & 10,88 & 10,58 & 0,878 \\
\hline Albumin (g/dl) & 3,9 & 0,14 & 3,892 & 0,23 & 0,923 \\
\hline Triglyceride $(\mathrm{mg}$ & 144,67 & 63,61 & 177,92 & 43,93 & 0,216 \\
\hline Cholesterin (mg/dl) & 148,44 & 34,95 & 163,25 & 27,1 & 0,309 \\
\hline Phosphat (mmol/l) & 2,42 & 0,12 & 2,35 & 0,2 & 0,310 \\
\hline $\mathrm{Ca}(\mathrm{mmol} / \mathrm{l})$ & 1,7 & 0,46 & 1,62 & 0,31 & 0,629 \\
\hline $\mathrm{P}^{*} \mathrm{Ca}(\mathrm{mmol} / \mathrm{l})$ & 50,92 & 12,27 & 46,9 & 8,47 & 0,414 \\
\hline $\mathrm{Hb}(\mathrm{mg} / \mathrm{dl})$ & 11,68 & 0,76 & 11,66 & 0,99 & 0,960 \\
\hline $\mathrm{mHN} \mathrm{AD}(\mathrm{mg} / \mathrm{c}$ & 64,59 & 11,6 & 66,09 & 13,2 & 0,786 \\
\hline mHN Red.(mg/d & 45,68 & 7,9 & 43,42 & 8,9 & 0,547 \\
\hline RRsyst. AD (mmHg) & 133,1 & 20,82 & 143 & 14,09 & 0,241 \\
\hline RR Diff. (mmHg) & 15,67 & 15,62 & 11,83 & 13,13 & 0,561 \\
\hline & & & & & MWU \\
\hline mGR (ml) & 6689 & 1916 & 7131 & 2895 & 0,859 \\
\hline & 472,2 & 657,7 & 542,5 & 665,6 & 0,774 \\
\hline Infektionen & 1,0 & 1,0 & 0,167 & 0,39 & 0,043 \\
\hline
\end{tabular}

Tabelle 7: Patientencharakteristika der Gruppen „,RDI $>=10^{“}$ und ,RDI $<10^{“}(\mathrm{x}=$ arithmetischer Mittelwert, SD = Standardabweichung, MWU = Mann-Whitney-U-Test $)$.

In der Gruppe der Patienten mit einem RDI < 10/h betrug das mittlere Alter 66,9 Jahre (s = 17,85), in der Gruppe der Patienten mit einem RDI >=10/h 71,2 Jahre (s = 11,37). Somit waren die Patienten mit Schlafapnoe im Mittel 4,3 Jahre älter.

Einen positiven Schlafapnoebefund hatte nur ein Patient aus der Gruppe unter 50 Jahren (49 J.). Ansonsten waren alle Patienten der Gruppe mit einem RDI >=10/h älter als 50 Jahre.

In der Gruppe der 51- bis 60 Jährigen hatte 1 von 2 Patienten einen positiven Schlafapnoebefund, in der Gruppe der 61- bis 70 Jährigen war es 1 von 4 Patienten, in der 
Gruppe der 71- bis 80 Jährigen waren es 4 von 6 Patienten und bei den Patienten über 80 Jahre wiesen 2 von 5 einen RDI >=10/h auf.

Das mittlere Dauer der Nierenersatztherapie der Patienten (,Dialysealter“) in der Gruppe mit RDI $<10$ betrug 2,78 Jahre $(s=2,18)$ und 5,33 Jahre $(s=4,77)$ für RDI >= 10 .

Das mittlere Gewicht lag bei 73,42 kg (s = 10,2) für RDI < 10 und bei $67,33 \mathrm{~kg}(\mathrm{~s}=10,12)$ für RDI >= 10. Die mittlere Körpergröße betrug für RDI <10 169,1 cm (s = 7,27) und für RDI $>=10165,4 \mathrm{~cm}(\mathrm{~s}=6,19)$.

Der durchschnittliche BMI unterschied sich mit 25,85 kg/m² $(\mathrm{s}=4,5)$ für RDI $<10$ und 24,52 $\mathrm{kg} / \mathrm{m}^{2}(\mathrm{~s}=2,93)$ für RDI $>=10$ nicht signifikant.

Bei den erhobenen Laborparametern, den Dialysedaten und Blutdruckwerten (siehe Tab. 7) ergab sich kein statistisch signifikanter Unterschied.

Signifikant hingegen unterschied sich die Häufigkeit der stationären Aufnahmen aufgrund von Infektionen in den letzten 2 Jahren vor Referenzdatum.

So wurden in den letzten 2 Jahren vor Referenzdatum in der Gruppe mit einem RDI < 10 (12 Patienten) insgesamt 2 Infektionen dokumentiert, die zu stationären Aufnahmen führten. In der Gruppe mit einem RDI >= 10 (9 Patienten) waren dies 9 stationäre Aufnahmen aufgrund von Infektionen.

\begin{tabular}{|l|c|c|c|c|c|}
\hline & \multicolumn{2}{|l|}{ RDI $>10$} & \multicolumn{2}{l|}{ RDI < 10 } & p - Wert \\
\hline $\begin{array}{l}\text { Risikofaktor/ } \\
\text { Begleiterkrankung }\end{array}$ & $\mathrm{n}$ & $\%$ & $\mathrm{n}$ & $\%$ & $\begin{array}{l}\text { Fisher's } \\
\text { exact test }\end{array}$ \\
\hline Männlich & 7 & 50 & 7 & 50 & 0,642 \\
\hline Weiblich & 2 & 28,6 & 5 & 71,4 & \\
\hline HTN & 8 & 88,9 & 12 & 100 & 0,429 \\
\hline DM & 4 & 44,4 & 3 & 25 & 0,397 \\
\hline HLP & 3 & 33,3 & 2 & 16,7 & 0,611 \\
\hline KHK & 4 & 44,4 & 3 & 25 & 0,397 \\
\hline CVI & 1 & 11,1 & 2 & 16,7 & $>0,99$ \\
\hline pAVK & 0 & 0 & 0 & 0 & 0 \\
\hline Progress KVE & 5 & 55,5 & 5 & 41,7 & 0,669 \\
\hline
\end{tabular}

Tabelle 8: Risikofaktoren und Begleiterkrankungen der Gruppen „RDI $>=10$ “ und „RDI $<10$ “ ( $\mathrm{n}=$ Anzahl, \% = Prozent). 
Beim Vergleich der Risikofaktoren für Kardiovaskuläre Erkrankungen (KVE) der Patienten ohne Schlafapnoe (,RDI < 10“) mit denen der Patienten mit Schlafapnoe (,RDI >=10“) ergab sich, dass in der Gruppe mit RDI < 10 alle 12 Patienten (100\%) einen vordiagnostizierten arteriellen Hypertonus hatten. Für RDI >= 10 waren es 8 der 9 Patienten $(88,9 \%)$. Zusätzlich führten wir vor und nach der Dialyse Messungen des Blutdrucks durch. Der Mittelwert des systolischen Blutdrucks vor Beginn der Dialyse lag bei $143 \mathrm{mmHg}$ (s = 14,09) für RDI < 10 und bei $133 \mathrm{mmHg}(\mathrm{s}=20,82)$ für RDI >=10. Der mittlere systolische Blutdruck nach Ende der Dialyse lag bei $131 \mathrm{mmHg}(\mathrm{s}=17,51)$ für RDI < 10 und bei 117 mmHg $(s=16,36)$ für RDI >=10. Die Differenz von Blutdruck vor und nach Dialyse betrug folglich im Mittel $12 \mathrm{mmHg}(\mathrm{s}=13,13)$ für RDI $<10$ und $16 \mathrm{mmHg}(\mathrm{s}=15,62)$ für RDI >= 10.

In der Gruppe mit einem RDI < 10 hatten 3 von 12 Patienten einen Diabetes mellitus, während in der Gruppe mit einem RDI >= 104 der 9 Patienten einen Diabetes mellitus hatten. Eine Hyperlipidämie lag bei 2 der 12 Patienten mit RDI < 10 vor. Aus der Gruppe RDI >= 10 waren 3 der 9 Patienten davon betroffen.

Bei der Betrachtung der vordiagnostizierten KVE zeigte sich, dass in der Gruppe RDI < 103 der 12 Patienten eine KHK hatten. Dies war für 4 der 9 Patienten mit einem RDI >= 10 der Fall. Die Prävalenz einer Cerebrovaskulären Insuffizienz (CVI) betrug 2 von 12 Patienten für RDI <10 und 1 von 9 Patienten für RDI >= 10 .

Eine pAVK war bei keinem der gescreenten Patienten bekannt.

Eine Verschlechterung/Progress der KVE innerhalb der letzten 2 Jahre vor Referenzdatum war bei 5 der 12 Patienten aus der Gruppe mit einem RDI < 10 und bei 5 der 9 Patienten aus der Gruppe mit einem RDI >= 10 beobachtet worden.

Somit war weder bei den kardiovaskulären Risikofaktoren, noch bei den kardiovaskulären Erkrankungen selbst ein statistisch signifikanter Unterschied zwischen den beiden Gruppen nachweisbar. 


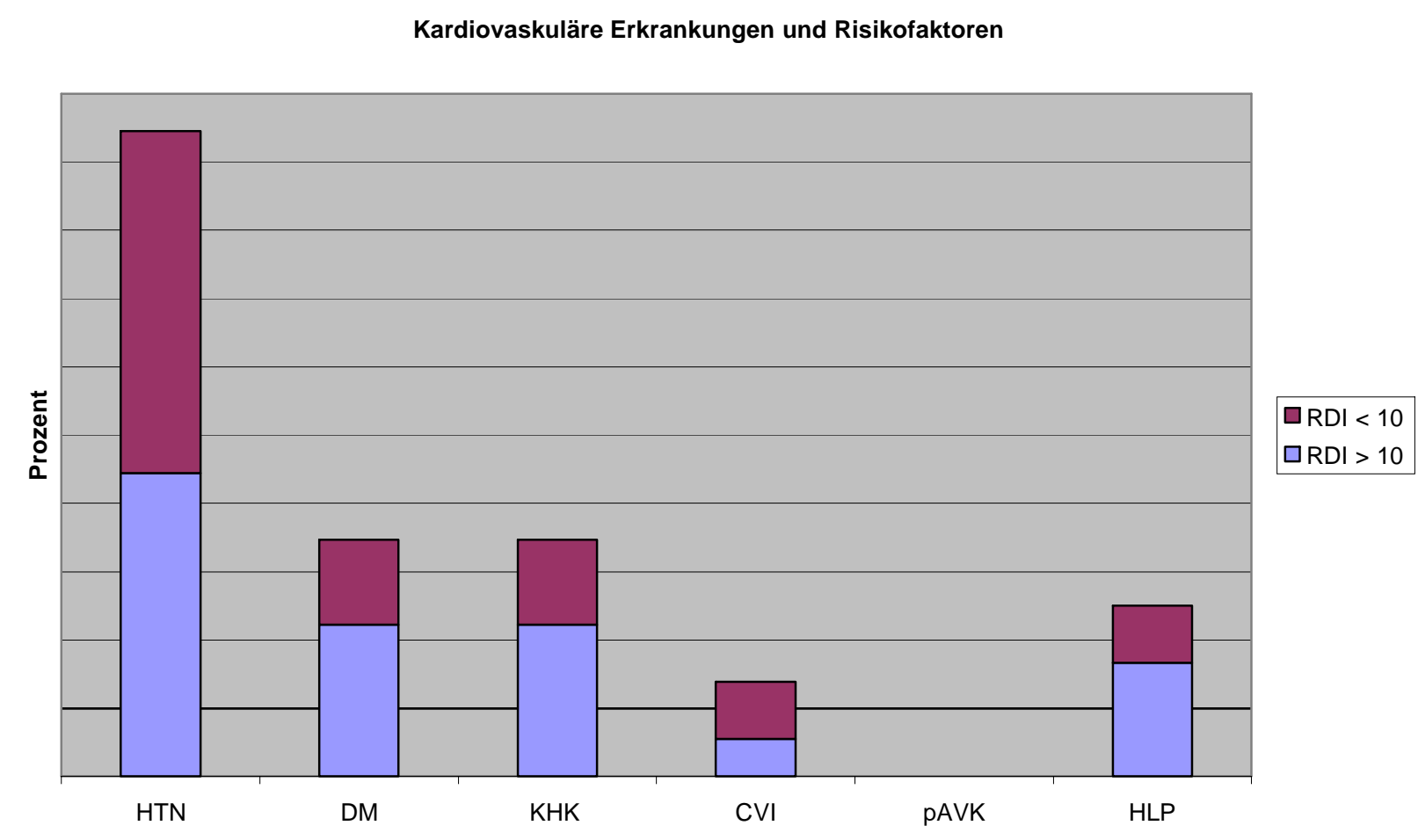

Abbildung 9: Kardiovaskuläre Erkrankungen und Risikofaktoren der Gruppen ,RDI >= 10“ und ,RDI < $10^{66}$

Beim Vergleich der für die chronische Niereninsuffizienz ursächlichen Erkrankungen zeigte sich Folgendes:

\begin{tabular}{|l|cc|cc|}
\hline Ursache der chron. Niereninsuffizienz & \multicolumn{2}{|l|}{ RDI > = 10 } & \multicolumn{2}{l|}{ RDI < 10 } \\
& Anzahl $n$ Prozent $\%$ & Anzahl n & Prozent $\%$ \\
\hline DM & 2 & 22,2 & 3 & 25 \\
\hline HTN & 0 & 0 & 2 & 16,7 \\
\hline NSAR & 2 & 22,2 & 0 & 0 \\
\hline GN & 0 & 0 & 3 & 25 \\
\hline SONST & 5 & 55,6 & 4 & 33,3 \\
\hline
\end{tabular}

Tabelle 9: Ursachen der chronischen Niereninsuffizienz der Gruppen ,,RDI >= 10“6 und ,,RDI $<11^{* 6}$ 
Die bestehende medikamentöse Behandlung wurde nicht unterbrochen.

Folgende Medikamente wurden eingenommen:

\begin{tabular}{|l|c|c|}
\hline Medikamentengruppe & $\begin{array}{l}\text { RDI >=10 } \\
\text { Anzahl n }\end{array}$ & $\begin{array}{l}\text { RDI < 10 } \\
\text { Anzahl n }\end{array}$ \\
\hline $\begin{array}{l}\text { Antihypertensiva } \\
\text { (insgesamt ) }\end{array}$ & 8 & 10 \\
\hline ACE-Hemmer & 0 & 1 \\
\hline AT-1 Antagonisten & 1 & 5 \\
\hline B-Blocker & 4 & 7 \\
\hline Ca- Antagonisten & 3 & 5 \\
\hline a-Blocker & 0 & 2 \\
\hline $\begin{array}{l}\text { Zentralwirksame } \\
\text { Antihypertensiva }\end{array}$ & 2 & 4 \\
\hline Diuretika & 5 & 5 \\
\hline Andere Antihypertensiva & 5 & 0 \\
\hline CSE-Hemmer & 4 & 5 \\
\hline Antikoagulanzien & 0 & 1 \\
\hline $\begin{array}{l}\text { Thrombozyten- } \\
\text { aggreationshemmer }\end{array}$ & 7 & 7 \\
\hline
\end{tabular}

Tabelle 10: Medikation zum Referenzdatum der Gruppen ,RDI >= 10“ und ,RDI $<10$ “

Bei der Auswertung der Fragebögen in Zusammenhang mit dem RDI, zeigte sich Folgendes:

\begin{tabular}{|l|c|c|c|c|c|}
\hline & \multicolumn{2}{|c|}{ RDI $>=10$} & \multicolumn{2}{l|}{ RDI $<10$} & p-Wert \\
\hline & $\mathrm{X}$ & SD & x & SD & MW Test \\
\hline Allgemeinbefinden & 4,33 & 2,29 & 3,5 & 1,73 & 0,427 \\
\hline ESS-Score & 5,89 & 4,23 & 4,58 & 3,12 & 0,591 \\
\hline & & & & & $\begin{array}{l}\text { Fisher's } \\
\text { exact test }\end{array}$ \\
\hline Schlafmedikamente & 3 & 33,3 & 3 & 25 & 1,0 \\
\hline Tagesmüdigkeit & 4 & 44,4 & 5 & 41,7 & 1,0 \\
\hline Unausgeschlafen & 7 & 77,8 & 5 & 41,7 & 0,184 \\
\hline Schlafstörungen & 7 & 77,8 & 6 & 50 & 0,364 \\
\hline Aufschrecken & 2 & 22,2 & 1 & 8,3 & 0,553 \\
\hline Schnarchen & 4 & 44,4 & 7 & 58,3 & 0,669 \\
\hline Atempausen & 1 & 11,1 & 1 & 8,3 & 1,0 \\
\hline Summe SA- Fragen & 28 & & 28 & & \\
\hline
\end{tabular}

Tabelle 11: Fragebogenergebnisse der Gruppen ,RDI >= 10“ und „,RDI $<10^{\circ}$ ( $(x=$ arithmetischer Mittelwert, SD = Standardabweichung, $n=$ Anzahl, MW Test $=$ Mann-Whitney-Test ) 
Für das Allgemeinbefinden (Skala von 1 bis 10) ergab sich ein mittlerer Wert von 3,5 (s = 1,73 ) in der Gruppe mit einem RDI < 10. Die Gruppe mit einem RDI >= 10 hatte mit 4,33 (s $=2,29$ ) einen etwas schlechteres mittleres Allgemeinbefinden angegeben.

Der mittlere Wert der ESS-Score war bei RDI $<10$ mit x = 4,58 $(\mathrm{s}=3,12)$ ebenfalls niedriger, als bei RDI $>=10$ mit $\mathrm{x}=5,89(\mathrm{~s}=4,23)$.

In der Gruppe mit einem RDI < 10 bestätigten jeweils 5 von 12 Patienten $(41,7 \%)$, an Tagesmüdigkeit bzw. morgendlicher Unausgeschlafenheit zu leiden. Hingegen litten in der Gruppe mit einem RDI >= 104 der 9 Patienten (44,4\%) an Tagesmüdigkeit bzw. 7 Patienten $(77,8 \%)$ an morgendlicher Unausgeschlafenheit. In beiden Gruppen wurde die regelmäßige Einnahme von Schlafmedikamenten von jeweils 3 Patienten bejaht. Dies entspricht $25 \%$ der Patienten mit einem RDI $<10$ und 33,3\% der Patienten mit einem RDI >= 10. 6 der 12 Patienten (50\%) für RDI < 10 und 7 der 9 Patienten (77,8\%) für RDI >= 10 gaben an, Schlafstörungen zu haben. Nur einer von 12 Patienten $(8,3 \%)$ für RDI < 10 berichtete von nächtlichem Aufschrecken. In der Gruppe mit einem RDI >= 10 waren es dagegen 2 von 9 Patienten (22,2\%). Für RDI < 10 war es 7 der 12 Patienten $(58,3 \%)$ bekannt, dass sie schnarchen würden. Bei RDI >= 10 war dies bei lediglich 4 der 9 Patienten $(44,4 \%)$ bekannt. Die für die Schlafapnoe typischen Atempausen während des Schlafes, wurden nach Aussage der Patienten nur bei jeweils einem Patienten aus jeder der zwei Gruppen beobachtet. Also bei $8,3 \%$ für RDI < 10 und bei $11,1 \%$ für RDI >= 10 .

Insgesamt zeigte die Auswertung der Fragebögen keinen statistisch signifikanten Unterschied zwischen den beiden Gruppen (RDI < 10 vs. RDI >=10). 


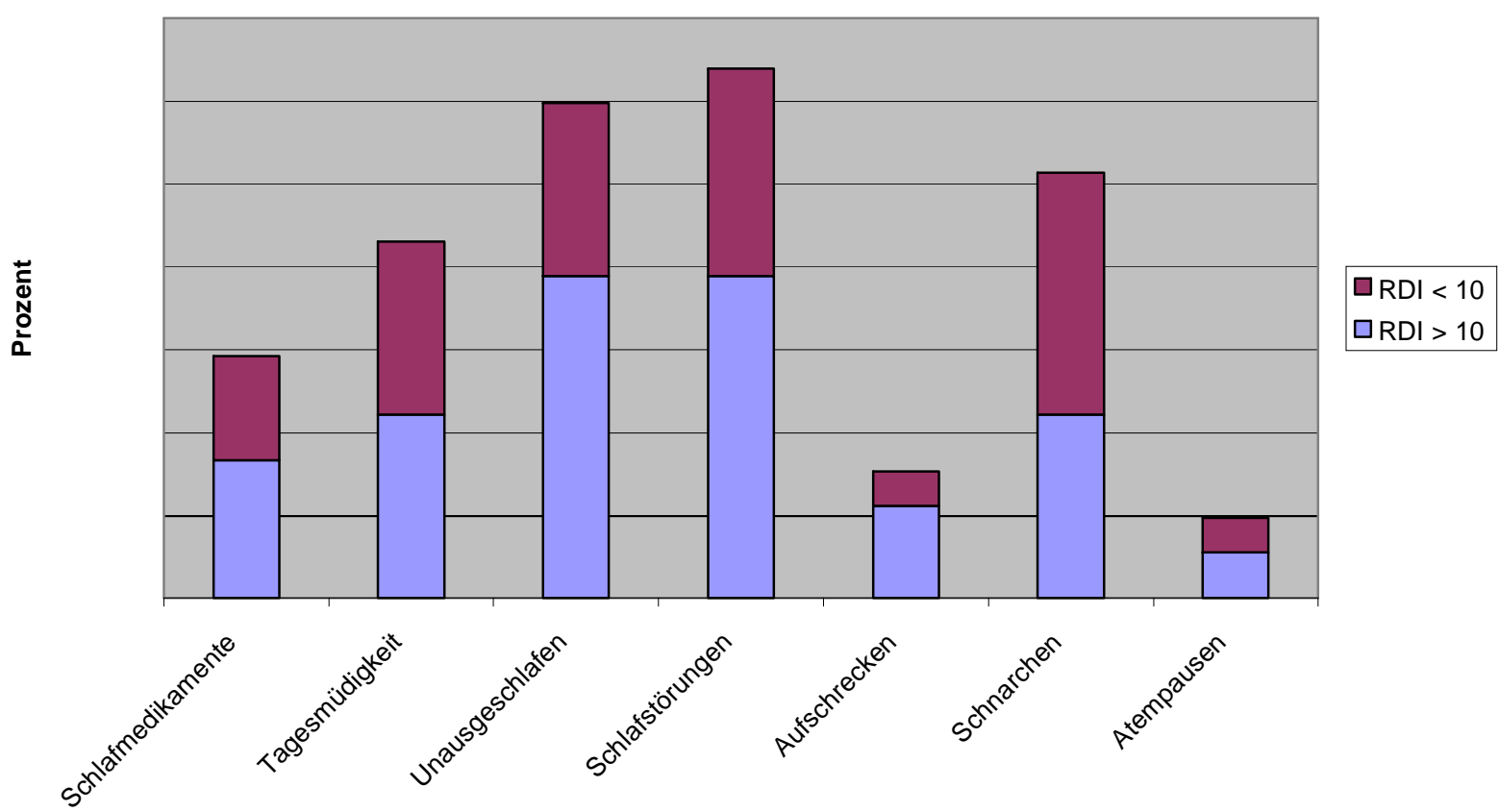

Abbildung 10: Ergebnisse des symptomspezifischen Fragebogens der Gruppen ,RDI >= 10“6 und ,,RDI < 106

\begin{tabular}{|l|l|l|}
\hline Exposition/Risikofaktor & OR & 95\% KI \\
\hline Progress KVE & 1,8 & $0,3-10,0$ \\
\hline Männl. Geschlecht & 2,5 & $0,4-17,5$ \\
\hline Diabetes mellitus & 2,4 & $0,4-15,3$ \\
\hline ESS > 8 & 5,5 & $0,5-65,2$ \\
\hline Tagesmüdigkeit & 1,1 & $0,2-6,4$ \\
\hline Schlafstörungen & 3,5 & $0,5-24,3$ \\
\hline Unausgeschlafen & 4,9 & $0,7-34,3$ \\
\hline Schlafmedikamente & 1,5 & $0,2-10,1$ \\
\hline Aufschrecken & 3,1 & $0,2-41,5$ \\
\hline Schnarchen & 0,6 & $0,1-3,3$ \\
\hline Atempausen & 1,4 & $0,1-25,4$ \\
\hline
\end{tabular}

Tabelle 12: Odds Ratio (OR) der ermittelten Expositionsfaktoren in Bezug auf einen RDI >= 10 (95\% KI: 95\% Konfidenzintervall). 


\section{Diskussion}

Zur Ermittlung der Prävalenz von SRBD bei Dialysepatienten und zur Klärung der Verwendbarkeit der üblichen Diagnosehilfen und - kriterien wurden die betreffenden Patienten der Station 1011 für eine Nacht mit einem ambulanten Screeninggerät überwacht. Zudem erfolgte die Evaluierung der Symptomatik mittels Fragebögen.

Zur Beurteilung des Einflusses der nächtlichen Atemstörungen auf die kardiovaskuläre Morbidität und Mortalität sowie auf die Häufigkeit des Auftretens von Infektionskrankheiten wurden die Patientenakten dahingehend ausgewertet.

Wir fanden bei $9(42,9 \%)$ von 21 nicht selektierten terminal niereninsuffizienten Patienten des untersuchten Kollektives einen RDI $>=10$.

Die Sensitivität und Spezifität dieses Kriteriums betragen nach den Ergebnissen von Ficker und Kollegen zur Evaluierung der Diagnosesicherheit eines Schlafapnoe - Syndroms mittels des von uns benutzten ambulanten Screening - Gerätes 0,83 und 0,95. Nach Abbildung 10 aus der genannten Studie werden somit bei dem Kriterium RDI >= 10 etwa $15 \%$ der betroffenen Patienten nicht erfasst.

\begin{tabular}{llllll}
\hline $\begin{array}{l}\text { Threshold } \\
\text { for } \text { AHI }_{\text {PsG }}\end{array}$ & Sensitivity & Specificity & PPV & NPV & Accuracy \\
\hline$\geq 5$ & 0.83 & 0.87 & 0.94 & 0.68 & 0.84 \\
$\geq 10$ & 0.83 & 0.95 & 0.96 & 0.81 & 0.88 \\
$\geq 20$ & 0.71 & 0.93 & 0.88 & 0.82 & 0.84 \\
$\geq 40$ & 0.61 & 1.00 & 1.00 & 0.88 & 0.90 \\
\hline & PPV = Pcoitive predictive value; NPV = negative predictive \\
value. & \\
\hline
\end{tabular}

Abbildung 11: Validitäts - Indices des ambulanten Screening - Gerätes Somno-check nach Ficker et al., 2001.

Die hohe Prävalenz unterscheidet sich im Vergleich zu nicht Dialysepatienten signifikant.

In einer Patientengruppe aus der Normalbevölkerung in der Altersgruppe von 50- 60 Jahren liegt die Prävalenz bei 10,6 \% (Männer und Frauen, n = 773) (Young et al., 1993). Die von uns untersuchten Patienten haben im Vergleich dazu somit eine signifikant höhere Prävalenz ( $\mathrm{p}=0,003$, Relatives Risiko ca. 4,1).

Der Vergleich unserer Daten mit den Studienergebnissen zu Prävalenzen der Schlafapnoe bei Dialysepatienten ergibt unterschiedliche Ergebnisse. Allgemein wurden Prävalenzen der Schlafapnoe bei Dialysepatienten von $11 \%$ - 89 \% ermittelt (Wadhwa et Mendelson., 1992; 
Walker et al., 1995; Burmann-Urbanek et al., 1995; Hallett et al., 1995; Kuhlmann et al., 2000).

So ermittelten Hallett et al. eine höhere Prävalenz von ca. $89 \%(n=9)$ als wir $(p=0,028)$. Dagegen war unsere Prävalenz signifikant höher $(\mathrm{p}=0,006)$ als die von Kuhlmann und Kollegen ermittelte Prävalenz von ca. $11 \%(n=55)$. Mit unseren Ergebnissen vergleichbare Werte $(\mathrm{p}=0,5)$ erhielten Wadhwa et Mendelson mit einer Prävalenz von 53,3 \% ( $\mathrm{n}=15)$.

Die große Differenz dieser Ergebnisse ist durch die unterschiedlichen Studiendesigns mit verschiedenen Diagnoseverfahren und - kriterien erklärbar.

Hinsichtlich der klinischen Diagnostik und Relevanz bestehen, zumindest im untersuchten Kollektiv erhebliche Unsicherheiten.

So ergab sich bei der Auswertung der ESS oder der „Schlafapnoe - Fragen“ keine Korrelation mit dem gemessenen RDI. Dies kontrastiert zu den Ergebnissen an „Normalpatienten“, da die ESS als geeignete Methode zur Identifizierung von Schlafapnoe-Patienten gilt (Johns, 1993) und in Untersuchungen der „Normalbevölkerung“ signifikant mit dem RDI korreliert (Johns, 1991).

Mit den „Schlafapnoe - Fragen“ wurden typische Symptome der Schlafapnoe (nach ICSD), wie Tagesmüdigkeit, morgendliche Unausgeschlafenheit, Schlafstörungen, nächtliches Aufschrecken aus dem Schlaf, Schnarchen und Atempausen während des Schlafes erfragt. In unserem untersuchten Patientenkollektiv waren diese Symptome nicht signifikant mit dem Schlafapnoe-Syndrom korreliert. Der Median der Summe der mit ,ja“ beantworteten „Schlafapnoe -Fragen“ beträgt in der Gruppe mit einem RDI >= $10(\mathrm{n}=9)$ 3,0. In der Gruppe mit einem RDI < $10(\mathrm{n}=12)$ beträgt der Median 2,5 ( $\mathrm{p}=0,27)$.

Dies lässt vermuten, dass die konventionellen Fragebögen wie ESS und die typischen Symptome der Schlafapnoe sich bei Dialysepatienten nicht als sicheres Screening-Verfahren zur Identifikation von Patienten mit SRBD eignen.

Ursache dafür ist vermutlich die Überschneidung der Schlafapnoe-Symptome mit den Beschwerden eines Dialysepatienten. Diese Beschwerden, wie beispielsweise Tagesmüdigkeit und Schlafstörungen sind Folge der Niereninsuffizienz, der Belastungen der chronischen Dialyse und der kardiovaskulären Folge- und Begleiterkrankungen der Dialysepatienten.

Im Gegensatz zu vorangegangenen Studien an „Nicht-Dialysepatienten“, konnte in der vorliegenden Untersuchung kein signifikanter Zusammenhang zwischen dem SchlafapnoeSyndrom und kardiovaskulären Risikofaktoren oder Erkrankungen gefunden werden. 


\subsection{Altersabhängigkeit}

Zwar waren die Dialysepatienten mit einer Schlafapnoe im Durchschnitt älter (71,2 +/- 11,37 vs. 66,9 +/- 17,85 Jahre), allerdings ohne statistische Signifikanz (p = 0,509), im Gegensatz zu anderen Studien. Andreas et al. untersuchten 1995 ein Patientenkollektiv, in dem die Patienten mit einem RDI > 10 signifikant älter waren, als die Patienten mit einem RDI < 10 (63,1 +/$3,5$ vs. $58,4+/-7,2$ Jahre; $p<0,002)$.

\subsection{Gewichtsabhängigkeit und Geschlecht}

Die festgestellten Assoziationen von Adipositas (Walsh et al., 1972) und dem männlichen Geschlecht (Young et al., 1993) mit der Schlafapnoe konnten bei Dialysepatienten nicht nachvollzogen werden.

Der BMI, als Indikator der Adipositas unterschied sich in unserem Patientenkollektiv nicht signifikant $\left(24,5+/-2,93\right.$ vs. $\left.25,9+/-4,5 \mathrm{~kg} / \mathrm{m}^{2} ; \mathrm{p}=0,424\right)$.

Während der BMI bei Nicht-Dialysepatienten einen signifikanten Risikofaktor darstellt (Andreas et al., 27,8 +/- 4,2 vs. $25,7+/-3,0 \mathrm{~kg} / \mathrm{m}^{2} ; \mathrm{p}<0.05$ ), zeigt sich bei den Dialysepatienten ein umgekehrter Trend.

Zwar war die Prävalenz der Schlafapnoe bei den Männern größer als in der Gruppe der Frauen (50\% vs. $28 \%)$, doch dieser Unterschied war nicht signifikant $(\mathrm{p}=0,34)$.

Die Prävalenz eines RDI >= 10 war in einer Patientengruppe aus der Normalbevölkerung in der Altergruppe 30-60 Jahre (Young et al., 1993) bei den untersuchten Männern deutlich höher als bei den Frauen (15\% vs. $5 \%$ ).

\subsection{Kardiovaskuläre Risikofaktoren}

Eine Hyperlipoproteinämie (HLP) war in der Patientengruppe mit einer Schlafapnoe etwas häufiger $(33,3 \%$ vs. $16,7 \%$; $=0,611)$. Eine signifikant höhere Häufigkeit einer HLP bei Schlafapnoe - Patienten konnte an unserem Patientenkollektiv somit nicht festgestellt werden. Die beschriebenen Zusammenhänge von Diabetes mellitus, arteriellem Hypertonus, koronarer Herzkrankheit (OR: 1,22, 95 \% KI: 0,93 - 1,59) und cerebrovaskulärer Insuffizienz (OR: 1,55, 95 \% KI: 0,96-2,5)(Shahar et al., 2001) mit dem Schlafapnoe - Syndrom konnten in der vorliegenden Untersuchung nicht signifikant bestätigt werden.

Die Prävalenzen des Diabetes mellitus und der KHK waren allerdings bei den Schlafapnoe Patienten etwas höher. 
Bei den von uns untersuchten Dialysepatienten hatten $44 \%$ der Patienten mit einem RDI >= 10 eines Diabetes mellitus. Hingegen litten nur $25 \%$ der Patienten mit einem RDI < 10 an einem Diabetes mellitus ( $\mathrm{p}=0,397)$.

Im Vergleich dazu zeigte eine Untersuchung von Reichmuth und Kollegen (2005; $\mathrm{n}=1387$ ) eine vergleichsweise hohe Prävalenz des Diabetes mellitus bei Schlafapnoe-Patienten. Insgesamt wurde bei $14,7 \%$ der Patienten mit einem RDI >= 15 ein Diabetes mellitus diagnostiziert. Dagegen war dies nur bei 2,8 \% der Patienten mit einem RDI $<5$ der Fall.

Eine koronare Herzkrankheit (KHK) war bei 7 der 21 untersuchten Dialysepatienten bekannt. Von den Patienten mit einem RDI >= 10 waren $44,4 \%$ an einer KHK erkrankt. In der Gruppe mit einem RDI < 10 waren dies mit $25 \%$ etwas weniger $(\mathrm{p}=0,397)$.

In einem Patientenkollektiv von 50 KHK - Patienten wurde im Vergleich dazu ein deutlicher Zusammenhang zwischen der Schlafapnoe und der KHK ermittelt (Andreas et al., 1996). Demnach hatten $50 \%$ dieser KHK-Patienten eine Schlafapnoe.

Ein relevanter Zusammenhang zwischen cerebrovaskulärer Insuffizienz (CVI) und der Schlafapnoe wurde von Bassetti und Aldrich (1999) ermittelt. 62,5\% der von ihnen untersuchten Patienten $(\mathrm{n}=80)$ mit einer CVI hatten eine polysomnographisch diagnostizierte Schlafapnoe. Dies war bei nur 12,5\% der ebenfalls untersuchten ,gesunden“ Kontrollgruppe $(\mathrm{n}=25)$ der Fall. Der mittlere AHI dieser beiden Gruppen unterschied sich signifikant ( $\mathrm{p}=$ 0,001).

In der von uns untersuchten Gruppe der Dialysepatienten konnte kein signifikanter Zusammenhang zwischen einer Schlafapnoe und CVI aufgezeigt werden. In der Schlafapnoe - Gruppe (RDI >=10) litten 11,1\% an einer CVI, in der Gruppe mit einem RDI < 10 waren es $16,7 \%(\mathrm{p}>0,99)$.

Ein signifikanter Zusammenhang zwischen einem arteriellen Hypertonus und der Schlafapnoe bei Dialysepatienten konnte ebenfalls nicht nachgewiesen werden. Es zeigte sich in beiden Gruppen (RDI >= 10 vs. RDI < 10) eine hohe Prävalenz des art. Hypertonus (88,9\% vs. 100 $\%, \mathrm{p}=0,429)$.

Demgegenüber hatten $23 \%$ eines Kollektivs von Hypertonikern $(n=68)$ einen AHI >=10/h. Und somit im Vergleich zur Kontrollgruppe der normotensiven Patienten, von denen keiner einen AHI >= 10 hatte, eine eindeutig höhere Prävalenz des Schlafapnoe-Syndroms (Worsnop et al., 1998). 
Insgesamt lassen sich die fehlenden Zusammenhänge der kardiovaskulären Risikofaktoren und Erkrankungen mit dem Schlafapnoe-Syndrom bzw. die fehlende Signifikanz mit der geringen Fallzahl erklären. Wie oben angegeben korellieren BMI, Geschlecht oder Blutdruck in unserer Patientengruppe nicht wie bei Nicht-dialysepflichtigen Patienten mit dem RDI. Trotz der Geringen Fallzahl legen die verglichenen Analysen mit veröffentlichten Studien jedoch nahe, dass es sich bei Dialysepatienten um ein von der "Normalbevölkerung" hinsichtlich des SRBD zu unterscheidendes Patientenkollektiv handelt. Natürlich kann ein Institutionseffekt durch Selektion bestimmter Patienten in einer universitären Einrichtung nicht ausgeschlossen werden. Leider war es innerhalb dieser Studie nicht möglich Patienten aus anderen Dialysezentren einzuschließen um diese Möglichkeit zu überpüfen. Soweit beurteilbar unterschieden sich die am Screening teilnehmenden Patienten nicht von denen, die eine Screening-Untersuchung abgelehnt hatten.

Im Falle der Adipositas und HLP könnte auch die im Durchschnitt geringe Prävalenz dieser Faktoren bei Dialysepatienten im Vergleich zur Normalbevölkerung ausschlaggebend sein.

Dies ist durch die Grunderkrankung und die Umstände der chronischen Dialyse bedingt.

Weder einer der ermittelten Laborparameter, noch einer der ermittelten Dialysedaten korrelierte signifikant mit dem RDI (Tabelle 7).

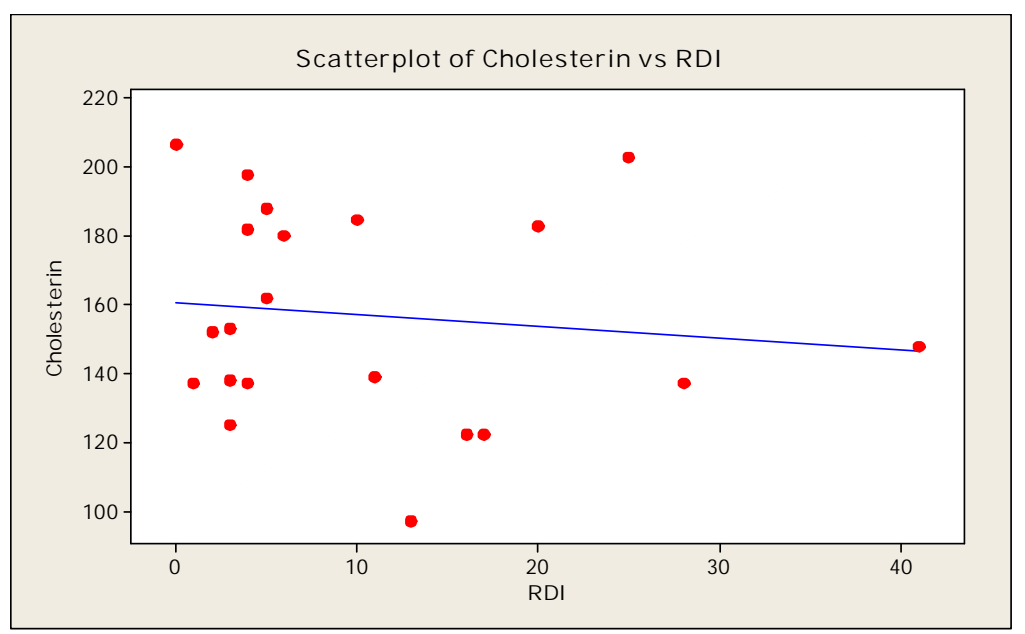

Abbildung 12: Korrelation von Cholesterin mit dem RDI 


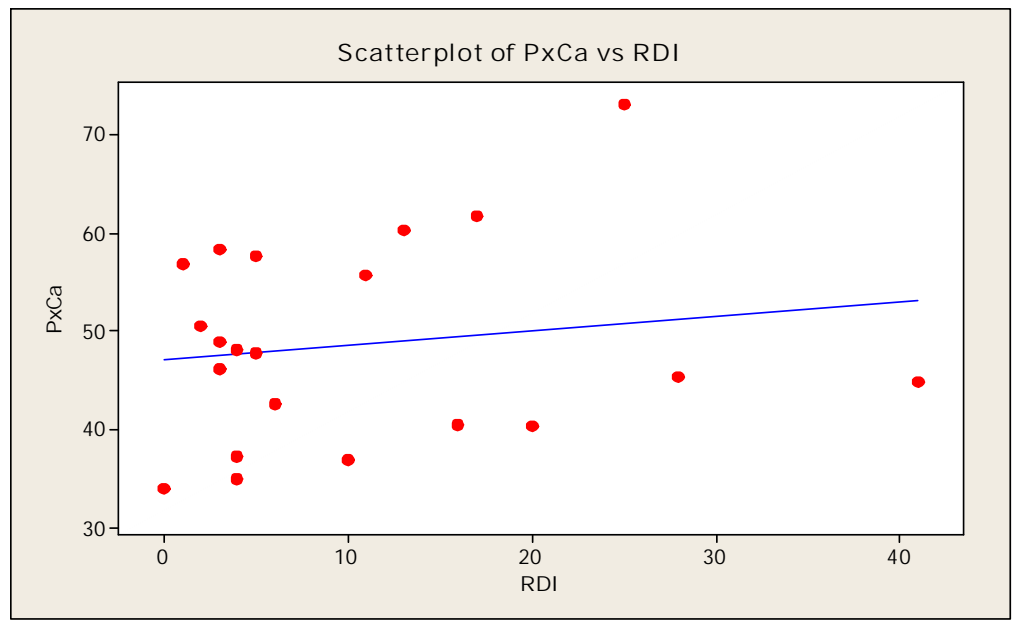

Abbildung 13: Korrelation von dem Phosphat-Calcium-Produkt mit dem RDI

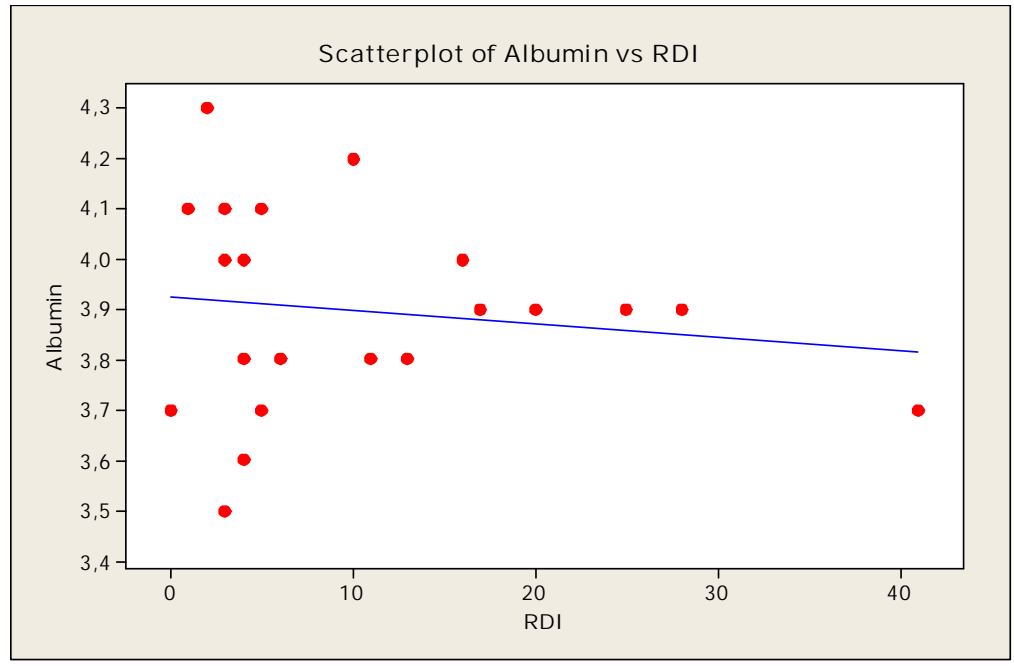

Abbildung 14: Korrelation von Albumin mit dem RDI

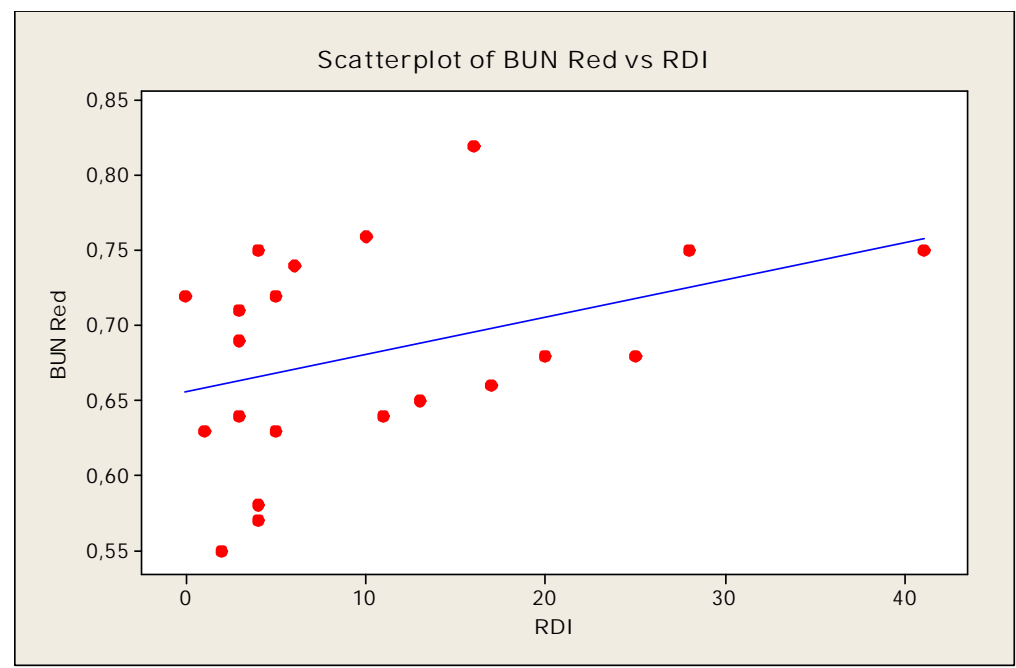

Abbildung 15: Korrelation der Harnstoff-N - Reduktion mit dem RDI 


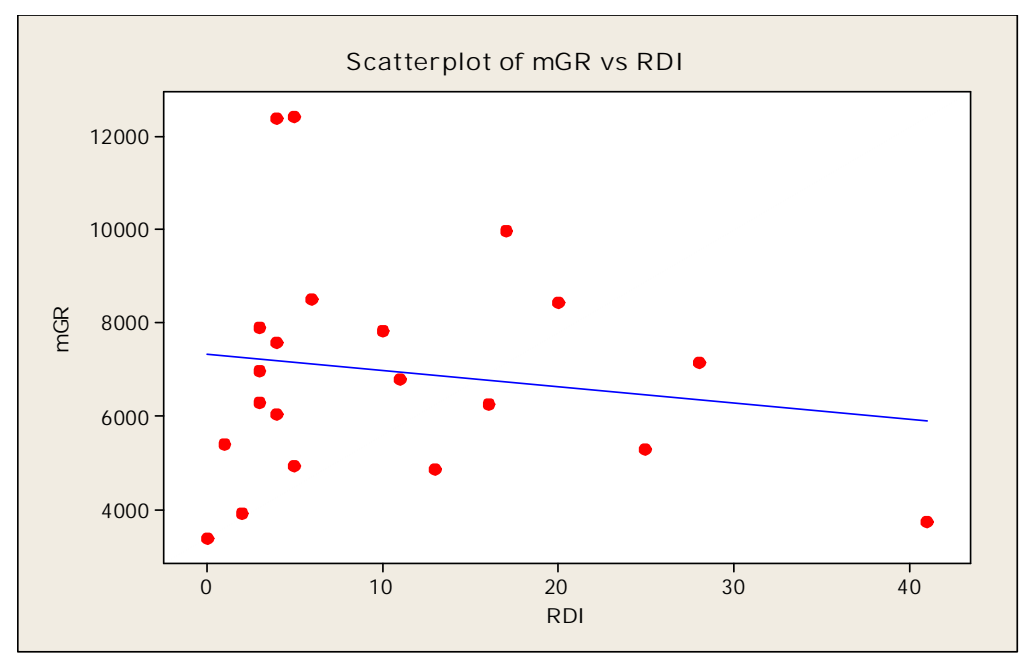

Abbildung 16: Korrelation der mittleren Gewichtsreduktion pro Woche mit dem RDI

Ein möglicher Zusammenhang von Dialysedosis und - effektivität mit der Schwere des Schlafapnoe-Syndroms konnte im untersuchten Patientenkollektiv nicht signifikant gezeigt werden. So unterscheiden sich die Werte der mittleren Gewichtsreduktion pro Woche und die Reduktion der Harnstoff $-\mathrm{N}$ Werte über den Zeitraum der Dialyse, als Indikator für die Einschätzung der konsequenten und effektiven Durchführung der Dialyse, nicht wesentlich. Dies steht nicht im Einklang mit den Ergebnissen von Hanly et Pierratos (2001), die signifikante Zusammenhänge der Effektivität der Hämodialyse mit der Prävalenz und Schwere der Schlafapnoe ermittelten. In der genannten Studie wurden Dialysepatienten von einem konventionellen Dialyserhythmus (3/Woche je 4 Stunden), ähnlich dem Rhythmus unseres Patientenkollektivs, auf nächtliche Hämodialyse (6-7 Tage/Woche je 8 Stunden) umgestellt.

Ergebnis waren signifikante Reduktionen von Apnoe-Hypopnoe-Index und Serum-KreatininKonzentration, sowie eine Erhöhung der Blutsauerstoffsättigung.

Hingegen konnte in der vorliegenden Untersuchung ein signifikanter Zusammenhang zwischen einer erhöhten Infektanfälligkeit und dem Schlafapnoe-Syndrom gefunden werden. In der Gruppe mit einem RDI >= 10 gab es im Mittel 1 Infektion pro Patient, die innerhalb der letzten 2 Jahre vor Referenzdatum zu einer stationären Aufnahme führte. In der Gruppe mit einem RDI < 10 waren es hingegen nur 0,167 Infektionen pro Patient (Median für RDI < $10=0$, Median für RDI >=10=1; $\mathrm{p}=0,043)$. 


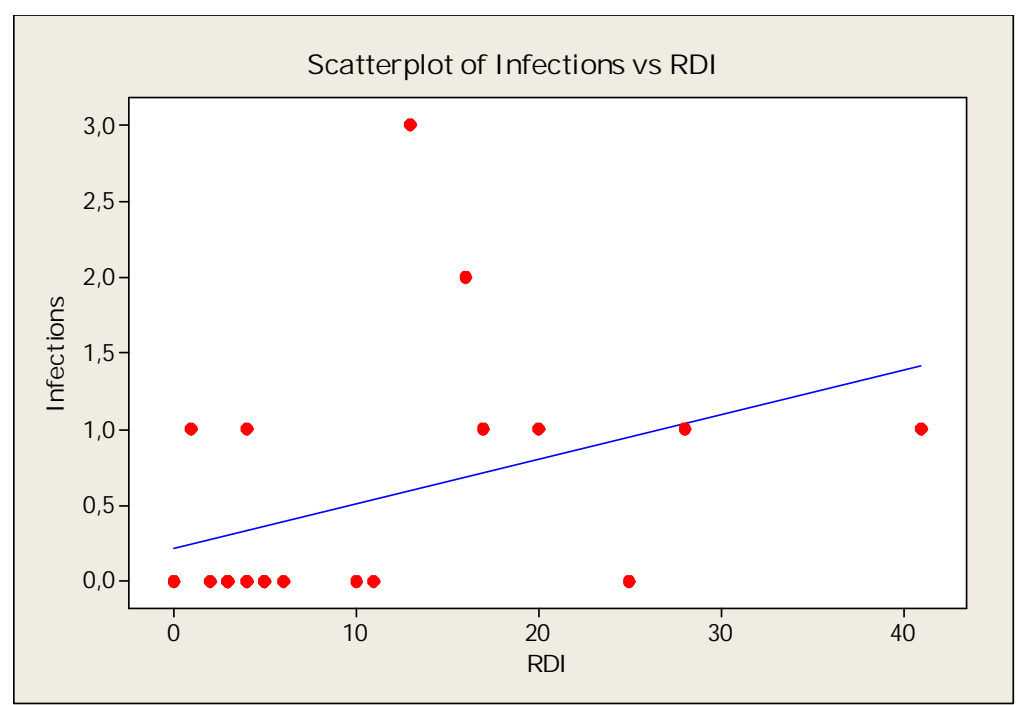

Abbildung 17: Korrelation von Anzahl der Infektionen mit dem RDI

Eine erhöhte Infektanfälligkeit der Dialysepatienten ist bekannt. Infektionen sind die zweithäufigste Todesursache von Dialysepatienten (Frei et Schober-Halstenberg, 2004). Erhöhte CRP - Werte werden als Hinweis auf ein hohes Mortalitätsrisiko bei Dialysepatienten angesehen (Iseki et al., 1999). Laut Zoccali et al. (2002 a) besteht ein Zusammenhang zwischen Pro - inflammatorischen Mediatoren und der Pathogenese der Schlafapnoe bei ESRD-Patienten. In Untersuchungen an Schlafapnoe-Patienten der „Normalbevölkerung“ wurden erhöhte Werte für TNF-a und IL-6 ermittelt (Vgontzas et al., 2000). Unabhängig davon wurden erhöhte Zytokinwerte bei Dialysepatienten gefunden (Rousseau et al., 2000).

Zusammenfassend ist festzustellen, dass die vorliegende Untersuchung auf eine hohe Prävalenz der Schlafapnoe bei Dialysepatienten im Vergleich zu Normalbevölkerung hinweist. Dies erhärtet die Ergebnisse früherer Studien.

Signifikante Zusammenhänge der Schlafapnoe mit den kardiovaskulären Risikofaktoren, wie männliches Geschlecht, arterieller Hypertonus, Hyperlipidämie, Diabetes mellitus und kardiovaskulären Erkrankungen, wie KHK, cerebrovaskuläre Insuffizienz und pAVK konnten nicht gezeigt werden. Frühere Studien haben diese Zusammenhänge allerdings für Nicht Dialysepatienten gezeigt. Diese Diskrepanz könnte durch die niedrige Fallzahl der vorliegenden Studie erklärt werden. Am ehesten liegt jedoch eine von den zuvor untersuchten Populationen zu unterscheidende Patientengruppe vor, bei der die etablierten Risikofaktoren anders zu bewerten sind. 
Abschließend konnte der mögliche Einfluß von SAS auf den Verlauf kardiovaskulärer Erkrankungen und Risikofaktoren bei Dialysepatienten aufgrund der niedrigen Fallzahl nicht geklärt werden.

Um z.B. eine Zunahme des kardiovaskulären Risikos von $10 \% / 2$ Jahre nachweisen zu können, wären bei der vorliegenden Prävalenz des Risikofaktors Schlafapnoe- Syndrom (RDI $>=10 ; 42,9 \%)$ und einer geschätzten gemischten Inzidenz von kardiovaskulären Ereignissen 3607 Patienten nötig (Power 0,8, p < 0,05).

Allerdings konnte ein signifikanter Zusammenhang zwischen einer erhöhten Infektanfälligkeit und dem Schlafapnoe-Syndrom ermittelt werden.

Die vorliegenden Ergebnisse verdeutlichen das Problem der Diagnosestellung einer Schlafapnoe bei Dialysepatienten. Die bei der Normalbevölkerung als Hinweise auf ein Schlafapnoe-Syndrom angewandten Assoziationen mit „typischen“ Symptomen (z.B. Tagesmüdigkeit, morgendliche Unausgeschlafenheit), Risikofaktoren (z.B. Alter, Geschlecht, Adipositas, HLP) und KVE (z.B. HTN, KHK, CVI) können für Dialysepatienten nicht in gleicher Weise gedeutet werden. Stattdessen bleiben die typischen Symptome differentialdiagnostisch häufig unberücksichtigt und werden stattdessen als Folge der Urämie, einer Depression oder im Rahmen der chronischen Erkrankung angesehen.

So sind konventionelle Fragebögen als Test auf Schlafstörungen bei Dialysepatienten aufgrund von Überschneidungen mit Symptomen der Grunderkrankung als ScreeningMethode für das SAS unseren Daten zufolge nicht effektiv.

Der Einsatz einfacher und effektiver Screening-Methoden ist daher zur Erkennung einer möglicherweise klinisch relevanten Schlafapnoe bei Dialysepatienten von entscheidender Bedeutung.

Das in der vorliegenden Untersuchung verwendete Screening-Verfahren mit dem Gerät „Somno-Check effort“ der Firma Weinmann ist ein anerkanntes ambulantes Verfahren bei der Diagnostik einer Schlafapnoe mit hoher Sensitivität $(0,83)$ und Spezifität $(0,95)$ (Ficker et al., 2001). Da das Screening nur für eine Nacht pro Patient durchgeführt wurde, sind die Ergebnisse der vorliegenden Untersuchung möglicherweise von einer Nacht - zu - Nacht Variabilität des Schlafes beeinflusst worden. Diese Variabilität kann sich beispielsweise in Unterschieden der Schlafposition oder infektbedingter Obstruktion der Atemwege manifestieren. Ein sehr leichter und unruhiger Schlaf durch die ungewohnte Schlafsituation mit Störungen des Schlafes durch die am Körper befindlichen Komponenten und eventuelle Alarmgeräusche des Screening-Gerätes können ebenfalls zur Beeinflussung der Ergebnisse geführt haben. 
Die Polysomnographie (PSG) gilt als Goldstandard in der Erkennung und Sicherung einer Schlafapnoe (ASDA, 1995). Allerdings ist die PSG sehr kosten - und zeitaufwendig. Die derzeitige Dichte an geeigneten Schlaflaboren ist in den meisten Gegenden Deutschlands noch nicht sehr hoch, so dass für viele betroffene Dialysepatienten eine aufwendige Anreise nötig wäre. Die Terminvergabe wäre bei steigenden Anfragen schwierig und mit langen Wartezeiten verbunden. Fraglich ist, ob die Dialysepatienten mit der ohnehin schon großen Belastung aufgrund der Grunderkrankung und deren aufwendiger Therapie, diese zusätzlichen Belastungen in Kauf nehmen, zumal der Langzeiteffekt der Schlafapnoe auf die Morbidität und die Mortalität der Dialysepatienten nicht bekannt ist. Hierzu wäre eine Studie mit mindestens 3607 Patienten (siehe oben) notwendig.

Allerdings wird die Schlafapnoe bei Nicht - Dialysepatienten als Risikofaktor für die kardiovaskuläre Morbidität und Mortalität angesehen (He et al., 1988 und Partinen et al. 2001). Somit macht die hohe Prävalenz eine vermehrte Aufmerksamkeit der behandelnden Ärzte der Dialysepatienten notwendig, da das Schlafapnoe-Syndrom als Risikofaktor für arteriellen Hypertonus, KHK, CVI und Herzinsuffizienz gilt und mit kardialen Arrhythmien und pulmonalem Hypertonus assoziiert ist. Da die Haupttodesursachen von Dialysepatienten kardiovaskulärer Herkunft sind (Frei et Schober-Halstenberg, 2004), bedeutet dies ein möglicherweise noch größeres Risiko für Dialysepatienten mit einer Schlafapnoe.

Hinzu kommt ein wahrscheinlicher Zusammenhang mit vermehrten Infekten und dem Schlafapnoe-Syndrom bei Dialysepatienten, bei denen nach QuaSi-Niere die infektionsbedingten Todesfälle an zweiter Stelle stehen.

Daher wäre der regelmäßige Einsatz einfacher aber zuverlässiger Screeningmethoden, beispielsweise mit einem ambulanten Screening-Gerät, möglicherweise die einzige Möglichkeit, Dialysepatienten mit SAS mit akzeptabler Sensitivität und Spezifität zu identifizieren. Nach der weiterführenden Diagnostik im Schlaflabor könnte die entsprechende Therapie eingeleitet werden. Aufgrund der ermittelten Daten bei Nicht - Dialysepatienten und den von uns ermittelten Daten (in Bezug auf die Komplikationen durch Infektionen) ist davon auszugehen, dass Lebensqualität, Lebenserwartung und die mit den kardiovaskulären Erkrankungen assoziierten Probleme der betreffenden Patienten möglicherweise durch eine Therapie der SAS verbessert werden könnten. Die Therapie mit einer nasalen CPAPBeatmung hat sich bei Dialysepatienten mit einer obstruktiven Schlafapnoe als effektiv erwiesen (Pressman et al., 1993). 
Zur Abklärung dieser vorgeschlagenen Vorgehensweise zur Diagnostik eines SchlafapnoeSyndroms bei Dialysepatienten sind hinsichtlich der Sensitivität des Screenings und der klinischen Relevanz allerdings größer angelegte Studien notwendig.

Es sollten darin weiterhin die für Dialysepatienten effektivsten diagnostischen Methoden überprüft bzw. ermittelt werden. Weiter ist es wichtig, die tatsächliche klinische Relevanz des Schlafapnoe-Syndroms bei Dialysepatienten zu evaluieren und, im Anschluss daran, die Signifikanz der Effektivität einer CPAP-Behandlung bei dieser speziellen Patientengruppe bezüglich des Schlafapnoe-Syndroms und der kardiovaskulären Morbidität und Mortalität sowie der Infektanfälligkeit zu ermitteln. 


\section{Zusammenfassung}

Dialysepatienten leiden überdurchschnittlich häufig am Schlafapnoe-Syndrom. Die typischen Symptome dieser schlafbezogenen Atemstörungen (SRBD) bleiben allerdings häufig unberücksichtigt, da diese sich mit den Symptomen der Niereninsuffizienz bzw. der Urämie überlappen. Des Weiteren ist die Prävalenz von kardiovaskulären Erkrankungen (KVE) bei Dialysepatienten hoch. Die KVE sind Hauptursache für die Mortalität der niereninsuffizienten Patienten. Danach folgen infektbedingte Todesfälle, wobei eine Assoziation von Infektionen und der Schlafapnoe vermutet wird.

In der Normalbevölkerung gilt das Schlafapnoe-Syndrom als Risikofaktor für eine erhöhte Inzidenz von kardiovaskulären Erkrankungen (KVE).

Ziel der vorliegenden Untersuchung war die Bestimmung der Prävalenz der Schlafapnoe bei den Dialysepatienten der Station 1011 des Universitätsklinikums Göttingen. Die an NichtDialysepatienten etablierten diagnostischen Verfahren (Fragebögen, Epworth Sleepiness Scale, ambulante Screeninggeräte) sollten an Dialysepatienten überprüft werden. Ein möglicher Zusammenhang zwischen kardiovaskulärer Morbidität und Mortalität sowie einer erhöhten Infektanfälligkeit bei Dialysepatienten mit SAS sollte erfasst werden.

Die eingeschlossenen Patienten wurden mit einem ambulanten Screeninggerät (,Somnocheck effort") in der Nacht nach der Dialyse gescreent. Es wurde ein Fragebogen mit den Patienten bearbeitet und alle relevanten Laborwerte, kardiovaskulären Diagnosen/Ereignisse und die relevanten Dialysedaten den Akten entnommen und ausgewertet.

Bei 9 der 21 Patienten (42,9 \%) ergab das Screening ein Schlafapnoe-Syndrom. Dies ist eine deutlich höhere Prävalenz als in der Normalbevölkerung.

Die Auswertung der Fragebögen ergab keine Korrelation $\mathrm{zu}$ den Ergebnissen des Schlafapnoe-Screenings. Es ist anzunehmen, dass die konventionellen Fragebögen mit den typischen Symptomen der Schlafapnoe und die ESS bei Dialysepatienten nicht erfolgversprechend sind. Folglich ist der Einsatz zuverlässiger ambulanter Screeninggeräte von Bedeutung, da die als Goldstandard geltende Polysomnographie (PSG) zurzeit nicht flächendeckend verfügbar ist.

In der vorliegenden Untersuchung an Dialysepatienten konnte kein signifikanter Zusammenhang zwischen dem Schlafapnoe-Syndrom und den kardiovaskulären Risikofaktoren und Erkrankungen ermittelt werden. 
Allerdings wurde eine signifikante Korrelation von dem Schlafapnoe-Syndrom mit erhöhter Infektanfälligkeit beobachtet. Da Infektionen die zweithäufigste Todesursache bei Dialysepatienten stellen, ist die erhöhte Infektneigung bei Niereninsuffizienz und Schlafapnoe von prognostischer Bedeutung.

Zur weiteren Abklärung der Relevanz des Schlafapnoe-Syndroms bei Dialysepatienten sind größer angelegte Studien notwendig. Ihre Aufgabe sollte es sein, die effektivsten diagnostischen Methoden der Schlafapnoe bei Dialysepatienten zu ermitteln. Die Zusammenhänge der KVE und der Infektneigung bei dieser Patientengruppe mit dem Schlafapnoe-Syndrom und deren tatsächliche klinische Bedeutung sollten weiter untersucht werden. Zudem sollte die Effektivität einer CPAP-Behandlung der Schlafapnoe bei Dialysepatienten vor allem in Bezug auf die kardiovaskulären Begleit - und Folgeerkrankungen bestimmt werden. 


\section{Anhang: Fragebogen}

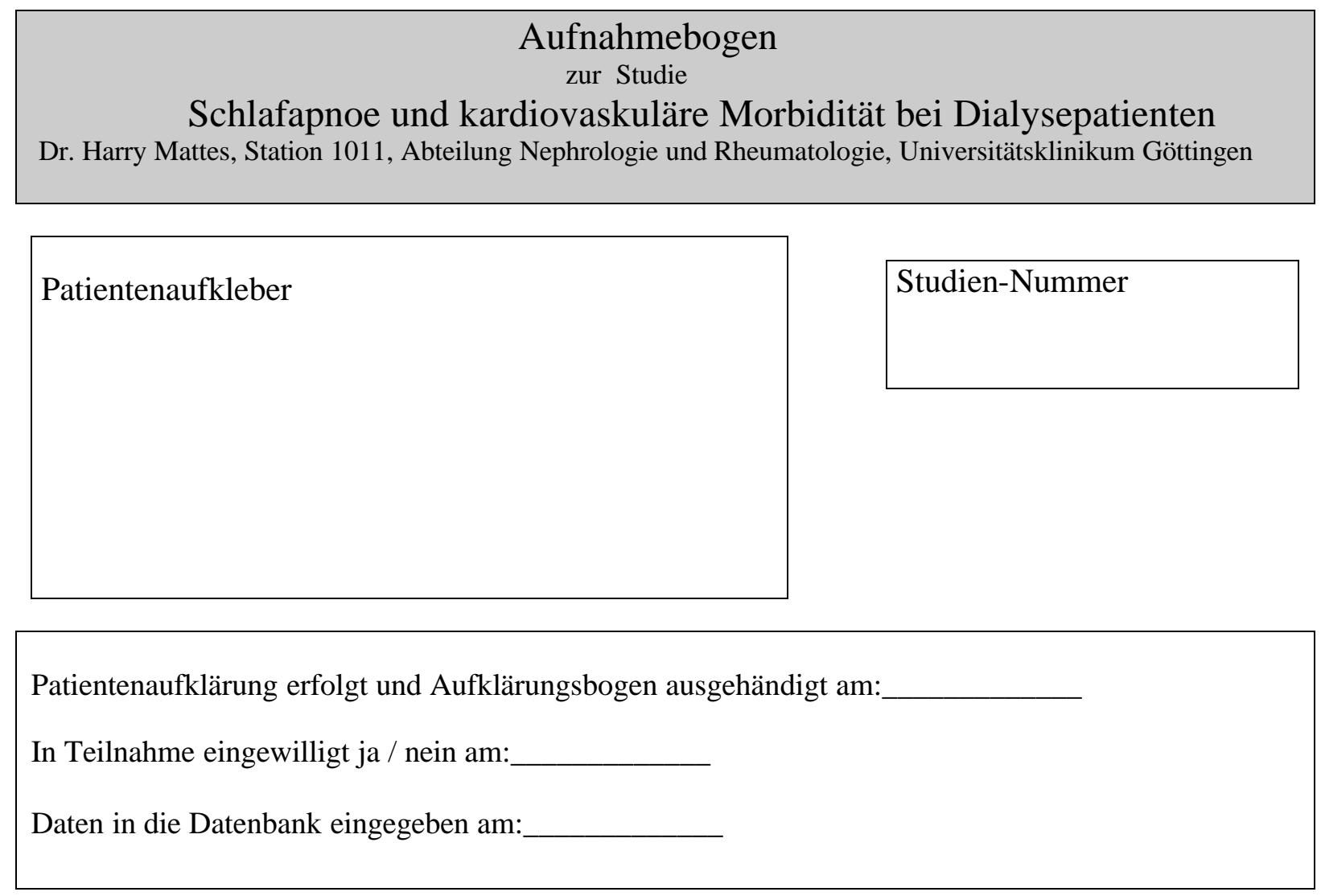

\section{Patientenbefragung am:}

Allgemeinbefinden ( 1 = sehr gut ....10 = sehr schlecht): 1---2---3---4---5---6---7---8---9---10

\section{Epworth Sleepiness Scale}

0 = würde niemals einnicken, 1 = geringe Wahrscheinlichkeit einzunicken, 2 = mittlere Wahrscheinlichkeit einzunicken, 3 = hohe Wahrscheinlichkeit einzunicken

\begin{tabular}{|l|l|}
\hline Im Sitzen lesen & \\
\hline Beim Fernsehen & \\
\hline Wenn Sie passiv (als Zuhörer) in der Öffentlichkeit sitzen & \\
\hline Als Beifahrer beim Autofahren während einer einstündigen Fahrt ohne Pause & \\
\hline Wenn sie sich am Nachmittag hingelegt haben, um auszuruhen & \\
\hline Wenn Sie sitzen und sich mit jemandem unterhalten & \\
\hline Wenn Sie nach dem Mittagessen (ohne Alkohol) ruhig dasitzen & \\
\hline Wenn Sie als Fahrer eines Autos verkehrsbedingt einige Minuten anhalten müssen & \\
\hline Summe = ESS - Score & \\
\hline
\end{tabular}


Schlafapnoe - Fragen

\begin{tabular}{|l|l|l|}
\hline & Ja & Nein \\
\hline Tagesmüdigkeit & & \\
\hline Schlafstörung & & \\
\hline Unausgeschlafen & & \\
\hline Aufschrecken & & \\
\hline Atempausen & & \\
\hline Schnarchen & & \\
\hline Schlafmedikamente & & \\
\hline
\end{tabular}

Studienindikation zum Schlafapnoe - Screening 


\section{Literaturverzeichnis}

AASM (American Academy of Sleep Medicine): The international classification of sleep disorders. Diagnostic and coding manual, Westchester/U.S.A. 1990

AASM (American Academy of Sleep Medicine) (1999): Sleep related breathing disorders in adults: recommendations for syndrome definition and measurement techniques in clinical research. The report of an American Academy of Sleep Medicine Task Force. Sleep 22, 667689

Alchanatis M, Tourkohoriti G, Kakouros S, Kosmas E, Podaras S, Jordanoglou JB (2001): Daytime pulmonary hypertension in patients with obstructive sleep apnea: the effect of continuous positive airway pressure on pulmonary hemodynamics. Respiration $\underline{68(6), 566-}$ 572

Amann K, Ritz E (1995): Strukturelle Veränderungen des Herzens bei Patienten mit chronischer Niereninsuffizienz. Spektrum Nephrol 6/7, 8-13

Anch AM, Remmers JE, Bunce H 3rd (1982): Supraglottic airway resistance in normal subjects and patients with occlusive sleep apnea. J Appl Physiol 53(5), 1158-1163

Andreas S, Schulz R, Haro W, Werner GS, Kreuzer H (1995): Sleep-related breathing disorders in patients with coronary heart disease. Dtsch Med Wochenschr 120(45), 1533-1537 Andreas S, Schulz R, Werner GS, Kreuzer H (1996): Prevalence of obstructive sleep apnoea in patients with coronary artery disease. Coron Artery Dis $\underline{7}(7), 541-545$

ASDA (American Sleep Disorders Association) (1995): Practice parameters for the treatment of snoring and obstructive sleep apnea with oral appliances. Sleep 18(6), 511-513

Auckley DH, Schmidt-Nowara W, Brown LK (1999): Reversal of sleep apnea hypopnea syndrome in end-stage renal disease after kidney transplantation. Am J Kidney Dis $\underline{34}(4), 739-744$

Auinger M, Wanke T, Merkle M, Lahrmann H, Kastenbauer T, Zwick H, Irsigler K (1995): Effect of autonomic neuropathy on ventilatory response to progressive hypercapnia in dialysis patients. Nephrol Dial Transplant $\underline{10}(6), 825-830$

Bady E, Achkar A, Pascal S, Orvoen-Frija E, Laaban JP (2000): Pulmonary arterial hypertension in patients with sleep apnoea syndrome. Thorax 55(11), 934-939

Balfors EM, Franklin KA (1994): Impairment of cerebral perfusion during obstructive sleep apneas. Am J Respir Crit Care Med 150(6 Pt 1), 1587-1591 
Basner RC, Ringler J, Garpestad E, Schwartzstein RM, Sparrow D, Weinberger SE, Lilly J, Weiss JW (1992): Upper airway anesthesia delays arousal from airway occlusion induced during human NREM sleep. J Appl Physiol 73(2), 642-648

Bassetti C, Aldrich MS (1999): Sleep apnea in acute cerebrovascular diseases: final report on 128 patients. Sleep $\underline{22}(2), 217-223$

Becker HF, Jerrentrup A, Ploch T, Grote L, Penzel T, Sullivan CE, Peter JH (2003): Effect of nasal continuous positive airway pressure treatment on blood pressure in patients with obstructive sleep apnea. Circulation 107(1), 68-73

Benca RM, Quintas J (1997): Sleep and host defenses: a review. Sleep 20(11), 1027-1037

Bennett LS, Barbour C, Langford B, Stradling JR, Davies RJ (1999): Health status in obstructive sleep apnea: relationship with sleep fragmentation and daytine sleepiness, and effects of continuous positive airway pressure treatment. Am J Respir Crit Care Med 159(6), 1884-1890

Benz RL, Pressman MR, Hovick ET, Peterson DD (1999): A preliminary study of the effects of correction of anemia with recombinant human erythropoietin therapy on sleep, sleep disorders, and daytime sleepiness in hemodialysis patients (The SLEEPO study). Am J Kidney Dis 34(6), 1089-1095

Berry RB, Kouchi K, Bower J, Prosise G, Light RW (1995): Triazolam in patients with obstructive sleep apnea. Am J Respir Crit Care Med 151(2 Pt 1), 450-454

Berthon-Jones M, Sullivan CE (1984): Ventilation and arousal responses to hypercapnia in normal sleeping humans. Appl Physiol 57(1), 59-67

Bixler EO, Vgontzas AN, Lin HM, Ten Have T, Rein J, Vela-Bueno A, Kales A (2001): Prevalence of sleep-disordered breathing in women: effects of gender. Am J Respir Crit Care Med. 163(3 Pt 1), 608-613

Bokinsky G, Miller M, Ault K, Husband P, Mitchell J (1995): Spontaneous platelet activation and aggregation during obstructive sleep apnea and its response to therapy with nasal continuous positive airway pressure. A preliminary investigation. Chest 108(3), 625-630

Bonora M, St John WM, Bledsoe TA (1985): Differential elevation by protriptyline and depression by diazepam of upper airway respiratory motor activity. Am Rev Respir Dis 131(1), 41-45

Bonsignore MR, Marrone O, Romano S, Pieri D (1994): Time course of right ventricular stroke volume and output in obstructive sleep apneas. Am J Respir Crit Care Med 149(1), 155-159

Borger L: Dialyse. Urban-Schwarzenberg, Monheim 1997 
Bowes G, Townsend ER, Bromley SM, Kozar LF, Phillipson EA (1981): Role of the carotid body and of afferent vagal stimuli in the arousal response to airway occlusion in sleeping dogs. Am Rev Respir Dis 123(6), 644-647

Bradley TD, Holloway RM, McLaughlin PR, Ross BL, Walters J, Liu PP (1992): Cardiac output response to continuous positive airway pressure in congestive heart failure. Am Rev Respir Dis $\underline{145}$ (2 Pt 1), 377-382

Buda AJ, Pinsky MR, Ingels NB Jr, Daughters GT 2nd, Stinson EB, Alderman EL (1979): Effect of intrathoracic pressure on left ventricular performance. N Engl J Med 301(9), 453-459

Burmann-Urbanek M, Sanner B, Laschewski F, Kreuzer I, Wiemann J, Konermann M, Sturm A (1995): Sleep disorders in patients with dialysis-dependent renal failure. Pneumologie 49 Suppl 1, 158-160

Cargill RI, Kiely DG, Lipworth BJ (1995): Adverse effects of hypoxaemia on diastolic filling

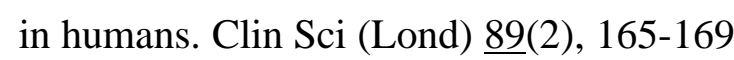

Carlson JT, Rangemark C, Hedner JA (1996): Attenuated endothelium-dependent vascular relaxation in patients with sleep apnoea. Hypertens $\underline{14}(5), 577-584$

Chadwick GA, Crowley P, Fitzgerald MX, O'Regan RG, McNicholas WT (1991): Obstructive sleep apnea following topical oropharyngeal anesthesia in loud snorers. Am Rev Respir Dis $\underline{143}$ (4 Pt 1), 810-813

Charlesworth JA, Kriketos AD, Jones JE, Erlich JH, Campbell LV, Peake PW (2005): Insulin resistance and postprandial triglyceride levels in primary renal disease. Metabolism $\underline{54}(6), 821-828$

Chesson AL Jr, Ferber RA, Fry JM, Grigg-Damberger M, Hartse KM, Hurwitz TD, Johnson S, Kader GA, Littner M, Rosen G (1997): The indications for polysomnography and related procedures. Sleep 20(6), 423-487

Chin K, Ohi M, Kita H, Noguchi T, Otsuka N, Tsuboi T, Mishima M, Kuno K (1996): Effects of NCPAP therapy on fibrinogen levels in obstructive sleep apnea syndrome. Am J Respir Crit Care Med 153(6), 1972-1976

Chin K, Nakamura T, Shimizu K, Mishima M, Nakamura T, Miyasaka M, Ohi M (2000): Effects of nasal continuous positive airway pressure on soluble cell adhesion molecules in patients with obstructive sleep apnea syndrome. Am J Med 109(7), 562-567

Chobanian AV, Bakris GL, Black HR, Cushman WC, Green LA, Izzo JL Jr, Jones DW, Materson BJ, Oparil S, Wright JT Jr; Joint National Committee on Prevention, Detection, Evaluation, and Treatment of High Blood Pressure. National Heart, Lung, and Blood Institute; 
National High Blood Pressure Education Program Coordinating Committee (2003): Seventh report of the Joint National Committee on Prevention, Detection, Evaluation, and Treatment of High Blood Pressure. Hypertension 42(6), 1206-1252

Cole P, Haight JS (1984): Posture and nasal patency. Am Rev Respir Dis 129(3), 351-354

Collard P, Pieters T, Aubert G, Delguste P, Rodenstein DO (1997): Compliance with nasal CPAP in obstructive sleep apnea patients. Sleep Med Rev 1(1):33-44

Craven JL, Rodin GM, Johnson L, Kennedy SH (1987): The diagnosis of major depression in renal dialysis patients. Psychosom Med $\underline{49}(5), 482-492$

Culebras A (2001): Restless leg syndrome. Diagnosis and treatment. Rev Neurol 32(3), 281283

Davies CW, Crosby JH, Mullins RL, Barbour C, Davies RJ, Stradling JR (2000): Casecontrol study of 24 hour ambulatory blood pressure in patients with obstructive sleep apnoea and normal matched control subjects. Thorax $\underline{55}(9), 736-740$

De Barbieri I, Zampieron A (2004): A descriptive analysis of sleep disturbances in patients with end-stage renal disease undergoing haemodialysis. EDTNA ERCA J 30(4) 213-216 Deegan PC, McNicholas WT (1995): Pathophysiology of obstructive sleep apnoea. Eur Respir J $\underline{8}(7), 1161-1178$

De Santo RM, Lucidi F, Violani C, Di Iorio BR (2005): Sleep disorders in hemodialyzed patients--the role of comorbidities. Int $J$ Artif Organs 28(6), 557-565 Dickens C: The posthumous papers of the Pickwick Club. Chapman and Hall, London 1837 Drager LF, Bortolotto LA, Lorenzi MC, Figueiredo AC, Krieger EM, Lorenzi-Filho G (2005): Early signs of atherosclerosis in obstructive sleep apnea. Am J Respir Crit Care Med $\underline{172}(5), 613-618$

Dyken ME, Somers VK, Yamada T, Ren ZY, Zimmerman MB (1996): Investigating the relationship between stroke and obstructive sleep apnea. Stroke 27(3), 401-407

El Bayadi S, Millman RP, Tishler PV, Rosenberg C, Saliski W, Boucher MA, Redline S

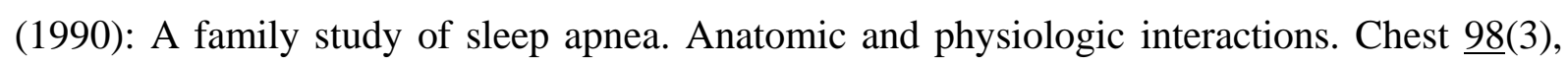
554-559

Evans RW (1991): Recombinant human erythropoietin and the quality of life of end-stage renal disease patients: a comparative analysis. Am J Kidney Dis 18(4 Suppl 1), 62-70

Fein AM, Niederman MS, Imbriano L, Rosen H (1987): Reversal of sleep apnea in uremia by dialysis. Arch Intern Med 147(7), 1355-1356 
Ficker JH, Wiest GH, Wilpert J, Fuchs FS, Hahn EG (2001): Evaluation of a portable recording device (Somnocheck) for use in patients with suspected obstructive sleep apnoea. Respiration 68(3), 307-312

Findley LJ, Levinson MP, Bonnie RJ (1992): Driving performance and automobile accidents in patients with sleep apnea. Clin Chest Med. 13(3),427-435

Fischer AQ, Chaudhary BA, Taormina MA, Akhtar B (1992): Intracranial hemodynamics in sleep apnea. Chest 102(5), 1402-1406

Flemons WW (2002): Clinical practice. Obstructive sleep apnea. N Engl J Med 347(7), 498504

Fletcher EC, DeBehnke RD, Lovoi MS, Gorin AB (1985): Undiagnosed sleep apnea in patients with essential hypertension. Ann Intern Med. 103(2), 190-195

Fletcher EC, Miller J, Schaaf JW, Fletcher JG (1987): Urinary catecholamines before and after tracheostomy in patients with obstructive sleep apnea and hypertension. Sleep 10(1), 3544

Fouke JM, Strohl KP (1987): Effect of position and lung volume on upper airway geometry. J Appl Physiol 63(1), 375-380

Franklin KA, Nilsson JB, Sahlin C, Naslund U (1995): Sleep apnoea and nocturnal angina. Lancet 345(8957), 1085-1087

Fraser CL, Arieff AI (1988): Nervous system complications in uremia. Ann Intern Med $\underline{109}(2), 143-153$

Frei U, Schindler R: Langzeitbetreuung von Patienten mit chronischer Nierenersatztherapie; in: Klinische Nephrologie; hrsg v. Koch KM; Urban\&Fischer, München, Jena 2000 a, 764778

Frei U, Schindler R: Nierentransplantation; in: Klinische Nephrologie; hrsg v. Koch KM; Urban\&Fischer, München, Jena 2000 b, 779-803

Frei U, Schober-Halstenberg HJ: Nierenersatztherapie in Deutschland. QuaSi-Niere Jahresbericht 2003/2004, Berlin 2004

Fung JW, Li TS, Choy DK, Yip GW, Ko FW, Sanderson JE, Hui DS (2002): Severe obstructive sleep apnea is associated with left ventricular diastolic dysfunction. Chest $\underline{121}(2)$, 422-429

Furst P (1989): Amino acid metabolism in uremia. J Am Coll Nutr $\underline{8}(4), 310-323$

Gami AS, Pressman G, Caples SM, Kanagala R, Gard JJ, Davison DE, Malouf JF, Ammash NM, Friedman PA, Somers VK (2004): Association of atrial fibrillation and obstructive sleep apnea. Circulation $\underline{110}(4), 364-367$ 
Gemeinsamer Bundesausschuss (2004): Zusammenfassender Bericht des Unterausschusses „Ärztliche Behandlung“ des G-BA über die Beratungen von 1998 bis 2004 zur Bewertung der Polygraphie und Polysomnographie im Rahmen der Differentialdiagnostik und Therapie der schlafbezogenen Atmungsstörungen gemäß § 135 Abs.1 SGB V . Dtsch Ärztebl 49, A 3370 A 3371

George CF (2001): Reduction in motor vehicle collisions following treatment of sleep apnoea with nasal CPAP. Thorax $\underline{56}(7), 508-512$

Grimm W, Koehler U, Fus E, Hoffmann J, Menz V, Funck R, Peter JH, Maisch B (2000): Outcome of patients with sleep apnea-associated severe bradyarrhythmias after continuous

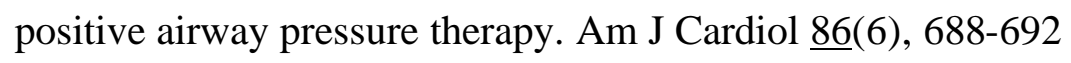

Grunstein RR, Ho KY, Sullivan CE (1991): Sleep apnea in acromegaly. Ann Intern Med $\underline{115}(7), 527-532$

Guilleminault C, Tilkian A, Dement WC (1976): The sleep apnea syndromes. Am Rev Med 27, $465-484$

Guilleminault C, Simmons FB, Motta J, Cummiskey J, Rosekind M, Schroeder JS, Dement WC( 1981): Obstructive sleep apnea syndrome and tracheostomy. Long-term follow-up experience. Arch Intern Med 141(8), 985-988

Guilleminault C, Connolly SJ, Winkle RA (1983): Cardiac arrhythmia and conduction disturbances during sleep in 400 patients with sleep apnea syndrome. Am J Cardiol 52(5), 490-494

Guilleminault C, Powell N, Bowman B, Stoohs R (1995): The effect of electrical stimulation on obstructive sleep apnea syndrome. Chest 107(1), 67-73

Hallett M, Burden S, Stewart D, Mahony J, Farrell P (1995): Sleep apnea in end-stage renal disease patients on hemodialysis and continuous ambulatory peritoneal dialysis. ASAIO J $\underline{41(3), 435-441}$

Hanly P, Pierratos A (2001): Improvement of sleep apnea in patients with chronic renal failure who undergo nocturnal hemodialysis. N Engl J Med 344(2), 102-107

Hanly P, Sasson Z, Zuberi N, Lunn K (1993): ST-segment depression during sleep in obstructive sleep apnea. Am J Cardiol 71(15), 1341-1345

Hanly P, Gabor JY, Chan C, Pierratos A (2003): Daytime sleepiness in patients with CRF: impact of nocturnal hemodialysis. Am J Kidney Dis $\underline{41}(2), 403-410$

Harbison J, O'Reilly P, McNicholas WT (2000): Cardiac rhythm disturbances in the obstructive sleep apnea syndrome: effects of nasal continuous positive airway pressure therapy. Chest $\underline{118}(3), 591-595$ 
He J, Kryger MH, Zorick FJ, Conway W, Roth T (1988): Mortality and apnea index in obstructive sleep apnea. Experience in 385 male patients. Chest 94(1), 9-14

Hershenson M, Brouillette RT, Olsen E, Hunt CE (1984) : The effect of chloral hydrate on genioglossus and diaphragmatic activity. Pediatr Res $\underline{18}(6)$ 516-519

Hughes JR (1980): Correlations between EEG and chemical changes in uremia. Electroencephalogr Clin Neurophysiol 48(5), 583-594

Hung J, Whitford EG, Parsons RW, Hillman DR (1990): Association of sleep apnoea with myocardial infarction in men. Lancet $\underline{36}$ (8710), $261-264$

Hwang JC, St John WM, Bartlett D Jr (1983): Respiratory-related hypoglossal nerve activity: influence of anesthetics. J Appl Physiol 55(3), 785-792

Ingbar DH, Gee JB (1985): Pathophysiology and treatment of sleep apnea. Annu Rev Med 36, 369-395

Iseki K, Tozawa M, Yoshi S, Fukiyama K (1999): Serum C-reactive protein (CRP) and risk of death in chronic dialysis patients. Nephrol Dial Transplant 14(8), 1956-1960

Issa FG, Sullivan CE (1982): Alcohol, snoring and sleep apnea. J Neurol Neurosurg Psychiatry 45(4), 353-359

Izzo JL Jr (1989): Sympathoadrenal activity, catecholamines, and the pathogenesis of vasculopathic hypertensive target-organ damage. Am J Hypertens $\underline{2}$ (12 Pt 2), 305-312

Javaheri S, Parker TJ, Liming JD, Corbett WS, Nishiyama H, Wexler L, Roselle GA (1998):

Sleep apnea in 81 ambulatory male patients with stable heart failure. Types and their prevalences, consequences, and presentations. Circulation 97(21), 2154-2159

Jennum P, Borgesen SE (1989): Intracranial pressure and obstructive sleep apnea. Chest 95, 279-283

Johns MW (1991): A new method for measuring daytime sleepiness: the Epworth sleepiness scale. Sleep 14(6), 540-545

Johns MW (1992): Reliability and factor analysis of the Epworth Sleepiness Scale. Sleep 15(4), 376-381

Johns MW (1993): Daytime sleepiness, snoring, and obstructive sleep apnea. The Epworth Sleepiness Scale. Chest 103(1), 30-36

Kales A, Bixler EO, Cadieux RJ, Schneck DW, Shaw LC 3rd, Locke TW, Vela-Bueno A, Soldatos CR (1984): Sleep apnoea in a hypertensive population. Lancet. 1984, 2, 1005-1008

Kaneko Y, Floras JS, Usui K, Plante J, Tkacova R, Kubo T, Ando S, Bradley TD (2003): Cardiovascular effects of continuous positive airway pressure in patients with heart failure and obstructive sleep apnea. N Engl J Med $\underline{348}(13), 1233-1241$ 
Kim J, In K, Kim J, You S, Kang K, Shim J, Lee S, Lee J, Lee S, Park C, Shin C (2004): Prevalence of sleep-disordered breathing in middle-aged Korean men and women. Am J Respir Crit Care Med. 170(10), 1108-1113

Kimmel PL (1989): Sleep disorders in chronic renal disease. J Nephrol 1, 59-65

Kimmel PL, Miller G, Mendelson WB (1989): Sleep apnea syndrome in chronic renal

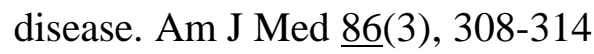

Koehler U, Dubler H, Glaremin T, Junkermann H, Lubbers C, Ploch T, Peter JH, Pomykaj T, von Wichert P (1991): Nocturnal myocardial ischemia and cardiac arrhythmia in patients with sleep apnea with and without coronary heart disease. Klin Wochenschr $\underline{69}(11), 474-482$

Koskenvuo M, Kaprio J, Telakivi T, Partinen M, Heikkila K, Sarna S (1987): Snoring as a risk factor for ischaemic heart disease and stroke in men. Br Med J (Clin Res Ed) 294(6563), 16-19

Kuhlmann U, Becker HF, Birkhahn M, Peter JH, von Wichert P, Schutterle S, Lange H (2000): Sleep-apnea in patients with end-stage renal disease and objective results. Clin Nephrol 53(6), 460-466

Laaban JP, Pascal-Sebaoun S, Bloch E, Orvoen-Frija E, Oppert JM, Huchon G (2002): Left ventricular systolic dysfunction in patients with obstructive sleep apnea syndrome. Chest $\underline{122}(4), 1133-1138$

Langevin B, Fouque D, Leger P, Robert D (1993): Sleep apnea syndrome and end-stage renal disease. Cure after renal transplantation. Chest 103(5), 1330-1335

Lavie L (2003): Obstructive sleep apnoea syndrome--an oxidative stress disorder. Sleep Med Rev $\underline{7}(1), 35-51$

Lavie L, Perelman A, Lavie P (2001): Plasma homocysteine levels in obstructive sleep apnea: association with cardiovascular morbidity. Chest $\underline{120}(3), 900-908$

Lavie P (1983): Incidence of sleep apnea in a presumably healthy working population: a significant relationship with excessive daytime sleepiness. Sleep $\underline{6}(4), 312-318$

Lavie P, Zomer J, Eliaschar I, Joachim Z, Halpern E, Rubin AH, Alroy G (1982): Excessive daytime sleepiness and insomnia. Arch Otolaryngol 108(6), 373-377

Lavie P, Herer P, Hoffstein V (2000): Obstructive sleep apnoea syndrome as a risk factor for hypertension: population study. BMJ $\underline{320(7233), 479-482}$

Littner M, Kushida CA, Hartse K, Anderson WM, Davila D, Johnson SF, Wise MS, Hirshkowitz M, Woodson BT (2000): Practice parameters for the use of laser-assisted uvulopalatoplasty: an update for 2000. Sleep 24(5), 603-619 
Livesley WJ (1981): Factors associated with psychiatric symptoms in patients undergoing chronic hemodialysis. Can J Psychiatry 26 (8), 562-566

Lonnemann G, Olbricht C, Nonnast-Danniel B, Brunkhorst R: Blutreinigungsverfahren bei terminaler Niereninsuffizienz; in: Klinische Nephrologie; hrsg v. Koch KM; Urban\&Fischer, München, Jena 2000, 750-752

Loube DI, Gay PC, Strohl KP, Pack AI, White DP, Collop NA (1999): Indications for positive airway pressure treatment of adult obstructive sleep apnea patients: a consensus statement. Chest $\underline{115}(3), 863-866$

Mall G (2000): Morphologie des Herzens bei Niereninsuffizienz und Hypertonie. Nieren- und Hochdruckkrankheiten $\underline{58}$, 197-201

McNicholas WT, Tarlo S, Cole P, Zamel N, Rutherford R, Griffin D, Phillipson EA (1982):

Obstructive apneas during sleep in patients with seasonal allergic rhinitis. Am Rev Respir Dis $\underline{126}(4), 625-628$

McNicholas WT, Bowes G, Zamel N, Phillipson EA (1984): Impaired detection of added inspiratory resistance in patients with obstructive sleep apnea. Am Rev Respir Dis 129(1), 4548

McQueen DS (1983): Opioid peptide interactions with respiratory and circulatory systems. Br Med Bull 39(1), 77-82

Merrill JP, Murray JE, Harrison JH, Guild WR (1956): Successful homotransplantations of the human kidney between identical twins. J Am Med Assoc 160(4), 277-82

Moldofsky H, Krueger JM, Walter J, Dinarello CA, Lue FA, Quance G, Oreopoulos DG (1985): Sleep-promoting material extracted from peritoneal dialysate of patients with endstage renal disease and insomnia. Perit Dial Bull $\underline{5}, 189-193$

Mooe T, Franklin KA, Holmstrom K, Rabben T, Wiklund U (2001): Sleep-disordered breathing and coronary artery disease: long-term prognosis. Am J Respir Crit Care Med $\underline{164}(10$ Pt 1), 1910-1913

Morgan BJ, Crabtree DC, Palta M, Skatrud JB (1995): Combined hypoxia and hypercapnia evokes long-lasting sympathetic activation in humans. J Appl Physiol 79(1), 205-213

Mucsi I, Molnar MZ, Rethelyi J, Vamos E, Csepanyi G, Tompa G, Barotfi S, Marton A, Novak M (2004): Sleep disorders and illness intrusiveness in patients on chronic dialysis. Nephrol Dial Transplant 19(7), 1815-1822

Mysliwiec M, Borawski J, Naumnik B, Rydzewska-Rosolowska A (2004): Endothelial dysfunction, atherosclerosis and thrombosis in uremia - possibilities of intervention. Akad Med Bialymst 49, 151-156 
Narkiewicz K, Montano N, Cogliati C, van de Borne PJ, Dyken ME, Somers VK (1998): Altered cardiovascular variability in obstructive sleep apnea. Circulation $\underline{98}(11), 1071-1077$ Netzer N, Werner P, Jochums I, Lehmann M, Strohl KP (1998): Blood flow of the middle cerebral artery with sleep-disordered breathing: correlation with obstructive hypopneas. Stroke $\underline{29}(1), 87-93$

Neumann C, Martinez D, Schmid H (1995): Nocturnal oxygen desaturation in diabetic patients with severe autonomic neuropathy. Diabetes Res Clin Pract 28 (2), 97-102

Newman AB, Nieto FJ, Guidry U, Lind BK, Redline S, Pickering TG, Quan SF; Sleep Heart Health Study Research Group (2001): Relation of sleep-disordered breathing to cardiovascular disease risk factors: the Sleep Heart Health Study. Am J Epidemiol 154(1), 5059

Nieto FJ, Young TB, Lind BK, Shahar E, Samet JM, Redline S, D'Agostino RB, Newman AB, Lebowitz MD, Pickering TG (2000): Association of sleep-disordered breathing, sleep apnea, and hypertension in a large community-based study. Sleep Heart Health Study. JAMA $\underline{283}(14), 1829-1836$

Oksenberg A, Silverberg DS, Arons E, Radwan H (1997): Positional vs nonpositional obstructive sleep apnea patients: anthropomorphic, nocturnal polysomnographic, and multiple sleep latency test data. Chest 112(3), 629-639

Orr WC, Martin RJ (1981): Obstructive sleep apnea associated with tonsillar hypertrophy in adults. Arch Intern Med 141(8), 990-992

Orr WC, Males JL, Imes NK (1981): Myxedema and obstructive sleep apnea. Am J Med 70(5), 1061-1066

Palomaki H (1991): Snoring and the risk of ischemic brain infarction. Stroke 22(8), 10211025

Parfrey PS, Vavasour HM, Henry S, Bullock M, Gault MH (1988): Clinical features and severity of nonspecific symptoms in dialysis patients. Nephron $\underline{50}(2), 121-128$

Parker KP, Bliwise DL, Rye DB (2000): Hemodialysis disrupts basic sleep regulatory mechanisms: building hypotheses. Nurs Res $\underline{49}(6), 327-332$

Parsons DS, Harris DC (1997): A review of quality of life in chronic renal failure. Pharmacoeconomics $\underline{12}$ (2 Pt 1), 140-160

Partinen M, Jamieson A, Guilleminault C (1988): Long-term outcome for obstructive sleep apnea syndrome patients. Mortality. Chest 94(6), 1200-1204 
Peker Y, Kraiczi H, Hedner J, Loth S, Johansson A, Bende M (1999): An independent association between obstructive sleep apnoea and coronary artery disease. Eur Respir J 14(1), 179-184

Peker Y, Hedner J, Kraiczi H, Loth S (2000): Respiratory disturbance index: an independent predictor of mortality in coronary artery disease. Am J Respir Crit Care Med 162(1), 81-86

Peppard PE, Young T, Palta M, Dempsey J, Skatrud J (2000): Longitudinal study of moderate weight change and sleep-disordered breathing. JAMA 284(23), 3015-3021

Peppard PE, Young T, Palta M, Skatrud J (2000): Prospective study of the association between sleep-disordered breathing and hypertension. N Engl J Med 342(19), 1378-1384

Perks WH, Cooper RA, Bradbury S, Horrocks P, Baldock N, Allen A, Van't Hoff W, Weidman G, Prowse K (1980): Sleep apnoea in Scheie's syndrome. Thorax 35(2), 85-91

Phillips BG, Narkiewicz K, Pesek CA, Haynes WG, Dyken ME, Somers VK (1999): Effects of obstructive sleep apnea on endothelin-1 and blood pressure. J Hypertens 17(1), 61-66

Pressman MR, Benz RL, Schleifer CR, Peterson DD (1993): Sleep disordered breathing in ESRD: acute beneficial effects of treatment with nasal continuous positive airway pressure. Kidney Int $\underline{43}(5), 1134-1139$

Rangemark C, Hedner JA, Carlson JT, Gleerup G, Winther K (1995): Platelet function and fibrinolytic activity in hypertensive and normotensive sleep apnea patients. Sleep $\underline{18}(3), 188-$ 194

Reichmuth KJ, Austin D, Skatrud JB, Young T (2005): Association of sleep apnea and type II diabetes: a population-based study. Am J Respir Crit Care Med 172(12), 1590-1595

Reinhardt B, Krick G: Verfahrenstechnische Aspekte; in: Blutreinigungsverfahren; hrsg. v. Franz HE, Hörl WH; Thieme, Stuttgart, New York 1997, 34-38

Remmers JE, deGroot WJ, Sauerland EK, Anch AM (1978): Pathogenesis of upper airway occlusion during sleep. J Appl Physiol 44(6), 931-938

Riley R, Guilleminault C, Herran J, Powell N (1983): Cephalometric analyses and flowvolume loops in obstructive sleep apnea patients. Sleep $\underline{6}(4): 303-311$

Riley R, Powell NB, Guilleminault C (1993): Obstructive sleep apnea syndrome: a review of 306 consecutively treated surgical patients. Otolaryngol Head Neck Surg 108(2), 117-125

Ritz E (2005): The clinical management of hyperphosphatemia. J Nephrol 18, 221-228

Rivlin J, Hoffstein V, Kalbfleisch J, McNicholas W, Zamel N, Bryan AC (1984): Upper airway morphology in patients with idiopathic obstructive sleep apnea. Am Rev Respir Dis 129(3), 355-360 
Robinson TG, Carr SJ (2002): Cardiovascular autonomic dysfunction in uremia. Kidney Int 62(6), 1921-1932

Rousseau Y, Haeffner-Cavaillon N, Poignet JL, Meyrier A, Carreno MP (2000): In vivo intracellular cytokine production by leukocytes during haemodialysis. Cytokine $\underline{12}(5), 506-$ 517

Rundcrantz H (1969): Postural variations of nasal patency. Acta Otolaryngol 68(5), 435-443

Sajkov D, Cowie RJ, Thornton AT, Espinoza HA, McEvoy RD (1994): Pulmonary hypertension and hypoxemia in obstructive sleep apnea syndrome. Am J Respir Crit Care Med 149(2 Pt 1), 416-422

Sanner BM, Doberauer C, Konermann M, Sturm A, Zidek W (1997): Pulmonary hypertension in patients with obstructive sleep apnea syndrome. Arch Intern Med 157(21), 2483-2487

Sanner BM, Klewer J, Trumm A, Randerath W, Kreuzer I, Zidek W (2000): Long-term treatment with continuous positive airway pressure improves quality of life in obstructive sleep apnoea syndrome. Eur Respir J $\underline{16}(1), 118-122$

Schäfer H, Hasper E, Ewig S, Lüderitz B (1996): Schlafbezogene Atmungsstörungen: Etabliertes und Neues in Diagnostik und Therapie. Dtsch Aerztebl 93, A-2396

Schäfer H, Koehler U, Ploch T, Peter JH (1997): Sleep-related myocardial ischemia and sleep structure in patients with obstructive sleep apnea and coronary heart disease. Chest $\underline{111}(2), 387-393$

Schafer ME (1982): Upper airway obstruction and sleep disorders in children with craniofacial anomalies. Clin Plast Surg 9(4), 555-567

Schöller B, Kotterba B (2001): Entwicklung eines miniaturisierten Systems für den ambulanten Heimeinsatz zur Erstdiagnose von schlafbezogenen Atemstörungen. Infobörse Mikrosystemtechnik $\underline{15}$

Schulz R, Schmidt D, Blum A, Lopes-Ribeiro X, Lucke C, Mayer K, Olschewski H, Seeger W, Grimminger F (2000): Decreased plasma levels of nitric oxide derivatives in obstructive sleep apnoea: response to CPAP therapy. Thorax 55(12), 1046-1051

Shahar E, Whitney CW, Redline S, Lee ET, Newman AB, Javier Nieto F, O'Connor GT, Boland LL, Schwartz JE, Samet JM (2001): Sleep-disordered breathing and cardiovascular disease: cross-sectional results of the Sleep Heart Health Study. Am J Respir Crit Care Med 163(1), 19-25 
Shamsuzzaman AS, Winnicki M, Lanfranchi P, Wolk R, Kara T, Accurso V, Somers VK (2002): Elevated C-reactive protein in patients with obstructive sleep apnea. Circulation 105(21), 2462-2464

Shelton KE, Woodson H, Gay S, Suratt PM (1993): Pharyngeal fat in obstructive sleep apnea. Am Rev Respir Dis 148(2), 462-466

Shepard JW Jr (1985): Gas exchange and hemodynamics during sleep. Med Clin North Am $\underline{69}(6), 1243-1264$

Shepard JW Jr, Garrison MW, Grither DA, Dolan GF (1985): Relationship of ventricular

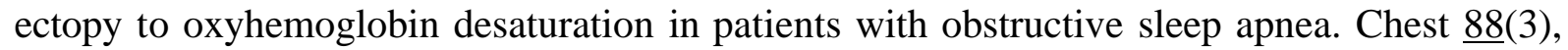
$335-340$

Sher AE, Schechtman KB, Piccirillo JF (1996): The efficacy of surgical modifications of the upper airway in adults with obstructive sleep apnea syndrome. Sleep 19(2), 156-177

Smith RP, Veale D, Pepin JL, Levy PA (1998): Obstructive sleep apnoea and the autonomic nervous system. Sleep Med Rev 2(2), 69-92

Somers VK, Dyken ME, Clary MP, Abboud FM (1995): Sympathetic neural mechanisms in obstructive sleep apnea. J Clin Invest $\underline{96}(4), 1897-1904$

Stradling JR, Thomas G, Warley AR, Williams P, Freeland A (1990): Effect of adenotonsillectomy on nocturnal hypoxaemia, sleep disturbance, and symptoms in snoring children. Lancet 335(8684), 249-253

Stradling JR, Crosby JH (1991): Predictors and prevalence of obstructive sleep apnoea and snoring in 1001 middle aged men. Thorax $\underline{46}(2), 85-90$

Strohl KP, Novak RD, Singer W, Cahan C, Boehm KD, Denko CW, Hoffstem VS (1994): Insulin levels, blood pressure and sleep apnea. Sleep 17(7), 614-618

Strub B, Schneider-Helmert D, Gnirss F, Blumberg A (1982): Sleep disorders in patients with chronic renal insufficiency in long-term hemodialysis treatment. Schweiz Med Wochenschr 112(23), 824-828

Sullivan CE, Issa FG, Berthon-Jones M, Eves L (1981): Reversal of obstructive sleep apnoea by continuous positive airway pressure applied through the nares. Lancet 1981, 1, 862-865

Taasan VC, Block AJ, Boysen PG, Wynne JW (1981): Alcohol increases sleep apnea and oxygen desaturation in asymptomatic men. Am J Med 71(2), 240-245

Vgontzas AN, Papanicolaou DA, Bixler EO, Hopper K, Lotsikas A, Lin HM, Kales A, Chrousos GP (2000): Sleep apnea and daytime sleepiness and fatigue: relation to visceral obesity, insulin resistance, and hypercytokinemia. J Clin Endocrinol Metab 85(3), 1151-1158 
Virga G, Stanic L, Mastrosimone S, Gastaldon F, da Porto A, Bonadonna A (2000): Hypercalcemia and insomnia in hemodialysis patients. Nephron $\underline{85}(1), 94-95$

Virolainen J, Ventila M, Turto H, Kupari M (1995): Effect of negative intrathoracic pressure on left ventricular pressure dynamics and relaxation. J Appl Physiol 79(2), 455-640

Wadhwa NK, Mendelson WB (1992): A comparison of sleep-disordered respiration in ESRD patients receiving hemodialysis and peritoneal dialysis. Adv Perit Dial $\underline{8}$, 195-198

Walker S, Fine A, Kryger MH (1995): Sleep complaints are common in a dialysis unit. Am J Kidney Dis $\underline{26}(5), 751-756$

Walsh RE, Michaelson ED, Harkleroad LE, Zighelboim A, Sackner MA (1972): Upper airway obstruction in obese patients with sleep disturbance and somnolence. Ann Intern Med $\underline{76}(2), 185-192$

Weiner D, Mitra J, Salamone J, Cherniack NS (1982): Effect of chemical stimuli on nerves supplying upper airway muscles. J Appl Physiol 52(3), 530-536

Wessendorf TE, Teschler H, Wang YM, Konietzko N, Thilmann AF (2000): Sleepdisordered breathing among patients with first-ever stroke. J Neurol 247(1), 41-47

White DP, Lombard RM, Cadieux RJ, Zwillich CW (1985): Pharyngeal resistance in normal humans: influence of gender, age, and obesity. J Appl Physiol 58, 365-371

Worsnop CJ, Naughton MT, Barter CE, Morgan TO, Anderson AI, Pierce RJ (1998): The prevalence of obstructive sleep apnea in hypertensives. Am J Respir Crit Care Med 157(1), 111-115

Wright J, Dye R: Systematic review on obstructive sleep apnoea: its effect on health and benefit of treatment. A report by the Yorkshire Collaborating Centre for Health Services Research. Leeds: University of Leeds, Nuffield Institute for Health, Leeds 1995

Yokoe T, Minoguchi K, Matsuo H, Oda N, Minoguchi H, Yoshino G, Hirano T, Adachi M (2003): Elevated levels of C-reactive protein and interleukin-6 in patients with obstructive sleep apnea syndrome are decreased by nasal continuous positive airway pressure. Circulation $\underline{107}(8), 1129-1134$

Young T, Palta M, Dempsey J, Skatrud J, Weber S, Badr S (1993): The occurrence of sleepdisordered breathing among middle-aged adults. N Engl J Med. 328(17), 1230-1235

Zoccali C, Ciccarelli M, Mallamaci F, Maggiore Q, Lotti M, Zucchelli GC (1987): Plasma met-enkephalin and leu-enkephalin in chronic renal failure. Nephrol Dial Transplant 1(4), 219-222 
Zoccali C, Benedetto FA, Tripepi G, Cambareri F, Panuccio V, Candela V, Mallamaci F, Enia G, Labate C, Tassone F (1998): Nocturnal hypoxemia, night-day arterial pressure changes and left ventricular geometry in dialysis patients. Kidney Int 53(4), 1078-1084

Zoccali C, Benedetto FA, Mallamaci F, Tripepi G, Candela V, Labate C, Tassone F (2001 a): Left ventricular hypertrophy and nocturnal hypoxemia in hemodialysis patients. Hypertens $\underline{19}(2), 287-293$

Zoccali C, Mallamaci F, Tripepi G (2001 b): Sleep apnea in renal patients. J Am Soc Nephrol $\underline{12}(12), 2854-2859$

Zoccali C, Mallamaci F, Tripepi G (2002 a): Nocturnal hypoxemia: a neglected cardiovascular risk factor in end-stage renal disease? Blood Purif 20(1), 120-123

Zoccali C, Mallamaci F, Tripepi G (2002 b): Nocturnal hypoxemia predicts incident cardiovascular complications in dialysis patients. J Am Soc Nephrol 13(3), 729-733

Zoccali C, Mallamaci F, Tripepi G (2003): Traditional and emerging cardiovascular risk factors in end-stage renal disease. Kidney Int Suppl 85, 105-110

Zorick F, Roth T, Kramer M, Flessa H (1980): Exacerbation of upper-airway sleep apnea by lymphocytic lymphoma. Chest 77(5), 689-690

Zwillich C, Devlin T, White D, Douglas N, Weil J, Martin R (1982): Bradycardia during sleep apnea. Characteristics and mechanism. J Clin Invest 69(6), 1286-1292 


\section{Danksagung}

Mein besonderer Dank gilt meinem Betreuer und dem ehemaligem Mitarbeiter der Abteilung Nephrologie und Rheumatologie der Universitätsmedizin Göttingen, Dr. med. Harry Mattes, für die Idee und Überlassung des interessanten Themas als Grundlage für die vorliegende Arbeit. Des Weiteren danke ich ihm für seine geduldige Unterstützung und Beratung bei der Durchführung und Niederschrift dieser Arbeit.

Ebenso möchte ich meinem Doktorvater und Leiter der Abteilung Nephrologie und Rheumatologie der Universitätsmedizin Göttingen, Prof. Dr. med. Gerhard-Anton Müller, für Korrektur und Begutachtung danken.

Weiterhin danke ich den Mitarbeitern und den Patienten der Station 1011 des Universitätsklinikums Göttingen für ihre freundliche Mitarbeit und Geduld bei der Durchführung.

Außerdem gilt mein Dank den Kollegen der Abteilung Kardiologie und Pneumologie für die freundliche Bereitstellung der benutzten ambulanten Screening-Geräte. 